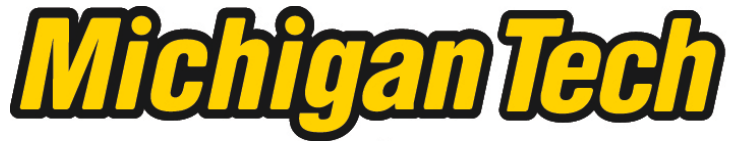 \\ Michigan Technological University Create the Future Digital Commons @ Michigan Tech
}

\section{Effect of microstructure and alloying elements on the resistance of fastener grade steels to hydrogen assisted cracking}

Nicholas E. Nanninga

Michigan Technological University

Follow this and additional works at: https://digitalcommons.mtu.edu/etds

Part of the Engineering Science and Materials Commons

Copyright 2005 Nicholas E. Nanninga

\section{Recommended Citation}

Nanninga, Nicholas E., "Effect of microstructure and alloying elements on the resistance of fastener grade steels to hydrogen assisted cracking ", Master's Thesis, Michigan Technological University, 2005.

https://doi.org/10.37099/mtu.dc.etds/24

Follow this and additional works at: https://digitalcommons.mtu.edu/etds

Part of the Engineering Science and Materials Commons 
Effect of Microstructure and Alloying Elements on the Resistance of

Fastener Grade Steels to Hydrogen Assisted Cracking

By

Nicholas E. Nanninga

A THESIS
Submitted in partial fulfillment of the requirements

For the degree of

MASTER OF SCIENCE IN MATERIALS SCIENCE \& ENGINEERING

Michigan Technological University

2005

Copyright @ Nicholas E. Nanninga 2005 
This thesis, "Effect of Microstructure and Alloying Elements on the Resistance of Fastener Grade Steels to Hydrogen Assisted Cracking,” is hereby approved in partial fulfillment of the requirements for the Degree of MASTER OF SCIENCE IN MATERIALS SCIENCE \& ENGINEERING

DEPARTMENT:

MATERIALS SCIENCE \& ENGINEERING

Signatures:

Thesis Advisor

Typewritten Name

Thesis Advisor

Typewritten Name

Department Chair

Typewritten Name

Date 


\begin{abstract}
Fastener grade steels with varying alloy contents and heat treatments were employed to measure changes in resistance to hydrogen assisted cracking. The testing procedure compared notched tension specimens fractured in air to threshold stress values obtained during hydrogen charging, utilizing a rising step load procedure. Bainitic structures improved resistance by $10-20 \%$ compared to tempered martensite structures. Dual phase steels with a tempered martensite matrix and 20\% ferrite were more susceptible and notch sensitive. High strength, fully pearlitic structures showed an improvement in resistance. Carbon content, per se, had no effect on the resistance of steel to hydrogen assisted cracking. Chromium caused a deleterious effect but all other alloying elements studied did not cause much change in hydrogen assisted cracking susceptibility.
\end{abstract}




\section{Table of Contents}

Signatures..................................................... II

Abstract...................................................... III

List of Figures............................................ VII

List of Tables............................................... IX

Chapter 1: Introduction......................................... 1

Chapter 2: Background.......................................... 4

2.1: Threaded Fasteners and Notched Fracture Mechanics......... 4

2.2: Hydrogen Induced Cracking (HIC)..................... 8

2.2.1: Mechanisms and Theories......................... 8

2.2.1.A: Troiano’s Decohesion Theory................. 9

2.2.2.B: Petch’s Surface Energy Reduction Theory...... 11

2.2.3.C: Beachem’s Model for Hydrogen-Assisted Cracking.................................. 12

2.2.2: Test methods for Hydrogen Induced Cracking Resistance...................................... 14

2.3: Steel Microstructures and Effects of Microstructures on HIC............................................. 17

2.3.1: Dual Phase Structure............................. 17

2.3.2: Martensite Versus Bainite........................ 22 
2.3.3: Alloying Element and Carbon Content Effects on HIC.

2.4: Fractography........................................ 29

2.5: Ferrite Crystallite Size.................................. 30

Chapter 3: Experimental Procedure.............................. 32

3.1: Notched Tension Sample Preparation and Heat Treatment.. 32

3.1.1: Sample Preparation.............................. 32

3.1.2: 10B21 Heat Treatment............................ 33

3.1.3: 1541 Heat Treatment.............................. 34

3.1.4: 1038 Heat Treatment.............................. 35

3.1.5: 4140, 1095 and 1065 Heat Treatment................ 37

3.1.5.A: Austempering.............................. 37

3.1.5.B: Quenching and Tempering.................. 38

3.1.6: 4037 Dual Phase Heat Treatment................... 39

3.2: Tension Testing...................................... 39

3.2.1: Rising Step Load (RSL) Procedure................... 39

3.3: Characterization......................................... 41

3.3.1: Hardness, Metallography and Fractography.......... 41

3.3.2: X-ray Diffraction............................... 42

Chapter 4: Results........................................ 44

4.1: Rising Step Load (RSL) Threshold Stress Results.......... 44 
4.1.1: Dual Phase......................................... 48

4.1.2: Effects of Alloying elements and Carbide Content on HIC............................................ 52

4.1.3: Austempered Vs. Quenched and Tempered........... 62

4.2: Ferrite Crystallite Size Analysis......................... 69

Chapter 5: Conclusions......................................... 72

Chapter 6: References.......................................... 73

Appendices................................................ $\quad 76$ 


\section{List of Figures}

2.1: Schematic illustration of a bolt showing stress concentration points where failure often occurs................................................

2.2: Geometry of a round bar with grooved notch typically used in stress concentration calculations

2.3: Schmatic representation of the fracture modes possible in steel when hydrogen cracking is involved

2.4: Schematic representation of the rising step load protocol used in finding the threshold load of the sample...

2.5: Schematic iron-carbon phase diagram showing the intercritical ferrite+austenite region used in dual phase heat treatments

2.6: Various heat treatment schedules used to produce a dual phased steel microstructure

2.7: Schematic representation of the formation of upper and lower bainite

2.8: Bainite and martensite start temperatures as a function of carbon concentration.

2.9: Illustration of fracture modes 30

3.1: Drawing and dimensions of the notched tensile sample.

3.2: Tempering curve for 10B21 alloy used in experiments

3.3: Tempering curve for alloy 1541 used in experiments

3.4: Images showing the heterogeneous microstructures of the 1038 samples heat treated through Jantom Precision.

3.5: Rack and samples used in the austempering of the 4140, 1095 and 1065 steel tensile samples.

4.1: Hydrogen charged and uncharged data for all alloys, heat treatments and strength levels

4.2: SEM images of CA and SA dual phase microstructures....

4.3: Bar charts displaying behavior of dual phase structures compared to quenched and tempered. 
4.4: Fractographs of 4037 step annealed dual phase steel

4.5: Plot showing the difference in uncharged fracture strength of the quenched and tempered carbon steels versus the alloyed steels.

4.6: SEM fracture surface of the 1038 uncharged specimens and optical image of 1095 specimen

4.7: Data indicating no change in threshold stress as carbon content is increased.

4.8: Plot showing the effects of the alloying content on HIC susceptibility

60

4.9: Fracture surfaces of quenched and tempered 4140 steel of hardness 466 HVN

4.10: Optical images of 4140 microstructures.

4.11: Hydrogen-induced cracking susceptibility of 4140 austempered and quenched and tempered steels

4.12: Hydrogen-induced cracking sucseptability of 1065 and 1095 austempered and quenched and tempered steel.

4.13: $S E M$ images of 1095 isothermally treated steels

4.14: SEM images of 1065 microstructures

4.15: 1095 pearlitic steel fracture surfaces.............................. 69

4.16: Differences in hardness as a function of particle size measurements...... $\quad 70$

4.17: Hydrogen-induced cracking susceptibility as a function of the ferrite crystallite size. 


\section{List of Tables}

3.1: Chemistry of fastener grade steel alloys used in testing................ 32

3.2: Loading profiles used in RSL testing of notched tensile specimens......... 41

4.1: Hardness value of all alloys tested............................... 45

4.2: Notch sensitivity calculations for austempered 1095 specimens............ 45 


\section{Chapter 1: Introduction}

Steel fasteners, such as screws, bolts and clamps, are utilized for a broad range of applications in the automotive industry. These steel fasteners are often exposed to environments where hydrogen can be absorbed, and the threads or notches on fasteners act to intensify the effects of hydrogen. Hydrogen that enters the steel is often picked up in the steel processing operations of acid cleaning and pickling or through electroplating of the fastener. The generation and absorption of hydrogen can also occur in service environments when a galvanic potential difference between the steel fastener and a damaged coating or another metal creates an electrochemical cell. The detrimental effects on steel that hydrogen can have are well known and have been studied extensively since the 1950's. Hydrogen may act to reduce the failure stress of the steel, act to limit the ductility within the steel or result in a combination of both. The failure of steel fasteners due to hydrogen attack may occur instantaneously upon loading or after a delayed period of time. This behavior makes it exceptionally difficult to predict and to design for resistance to hydrogen induced failures, and is extremely costly from the stand point of liability and repairs.

There are several mechanisms that have been proposed to explain the effect that hydrogen has on iron and steel. Some of the mechanisms that are reviewed are the surface energy reduction theory proposed by Petch [1], the decohesion theory proposed by Troiano [2] and a theory by Beachem [3] that hydrogen enhances each of the several normal fracture modes. Many different nomenclatures have been defined to label effects of hydrogen in steel due to different variables. These typically include stress corrosion cracking (SCC) and hydrogen embrittlement (HE). Hydrogen embrittlement is often used 
to describe failures due to hydrogen introduced into the steel during processing, or anytime before service. Fracture caused by hydrogen introduced into the steel during the corrosion reaction of iron is typically termed stress corrosion cracking. Other terms for hydrogen damage in steel are hydrogen induced cracking (HIC) or hydrogen assisted cracking (HAC). All these terms describe the process of atomic hydrogen being absorbed into steel and acting to reduce the strength of the material. Throughout this paper all mentioned terms are used interchangeably.

There has been a significant amount of research directed at the role that microstructures of steel play in resisting the harmful effects of hydrogen. Microstructures examined have included: pearlitic, martensitic and tempered martensite, bainitic, dual phase, austenitic and spheroidized. It is often accepted that martensite is most susceptible to hydrogen damage, but tempered martensite is least susceptible. Alloying elements, including carbon, may also change the behavior of steels charged with hydrogen.

Previous research conducted at Michigan Technological University studied the behavior of 4037 steel with tempered martensite and a dual phase martensite plus ferrite microstructure [4]. The results of this work supplement the present work, which deals with investigating the effects of isothermal, dual phase and conventional quench and tempering heat treatments in hydrogen assisted cracking. The goal of this research is to study the effects that a microstructure and alloying elements have on hydrogen induced cracking behavior. This was performed by measuring the hydrogen charged threshold fracture stress of notched steel specimens under a rising step load (RSL) tensile testing procedure. Typical fastener grade alloys with tempered martensite microstructures 
containing varying elements including: boron, carbon, manganese, molybdenum and chromium were to be compared. Also, common alloys were heat treated to compare quenched and tempered microstructures to dual phase or bainitic structures. 


\section{Chapter 2: Background}

\section{1: Threaded Fasteners and Notched Fracture Mechanics}

The significance of fasteners, such as screws, bolts and nuts, is often overlooked, but fasteners are vital mechanical latching systems for many applications, including power plant equipment, buildings and bridges, the aerospace industry, and of course the automotive industry. Stress corrosion cracking or hydrogen embrittlement susceptibility can often play a role in the design of steel fasteners, because in many applications fasteners are exposed to environments that could potentially degrade their properties. Before analyzing the effects the environment or hydrogen have on steel fasteners, it is important to understand some of the basic properties of a threaded fastener.

A threaded fastener can essentially be compared to a round bar, typically stressed in uniaxial tension, with several notches that act as stress risers [5]. A fastener usually fails at one of three locations: a thread root radius, the thread to shank radius, or the head to shank fillet [6]. Figure 2-1 shows a longitudinal view of a threaded fastener, highlighting the stress concentrations. The failure in these locations is due to stress concentrations that are localized where the radius is decreased. Notches and grooves create triaxial stress states that resist local plastic flow during loading and result in increased yield strengths and brittle fracture; whereas a non-grooved steel rod would exhibit ductile fracture [7]. For this reason, it is important to design steel fasteners that have adequate toughness and ductility [5]. 


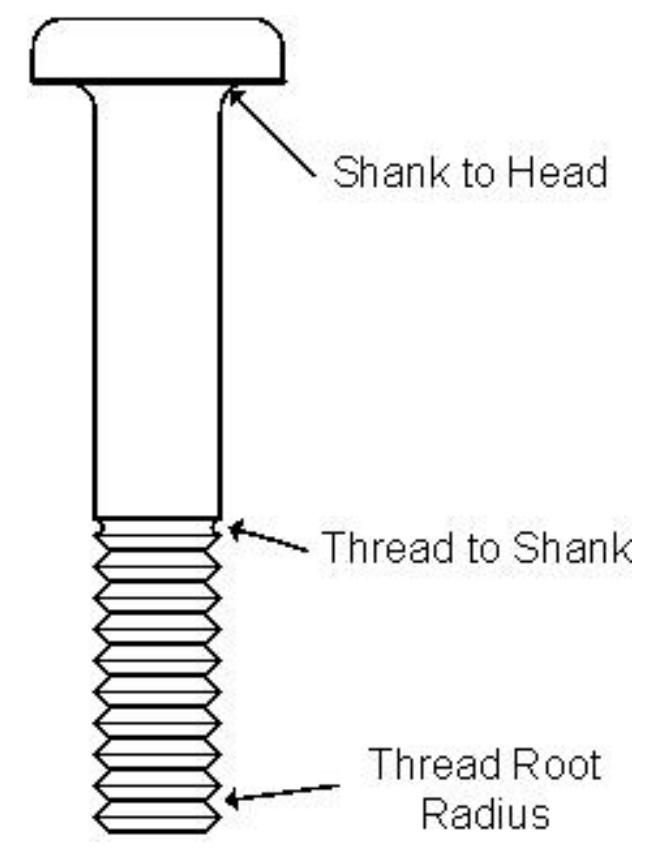

Figure 2-1: Schematic illustration of a bolt showing stress concentration points where failure often occurs.

The amount of threads that are engaged during the loading of the fastener will affect the stress concentration at the notch roots. If more threads are engaged, then the stress concentration is lower, due to an even distribution of the stresses [6]. Also, the first engaged thread on the fastener typically experiences the highest stress concentrations [7]. The analysis of a single grooved round bar is often adequate when theoretically calculating stress concentrations for threaded fasteners, because the one notch will experience a higher stress than each of a series of threads [6]. Figure 2-2 provides a drawing of a round bar with a single groove. 


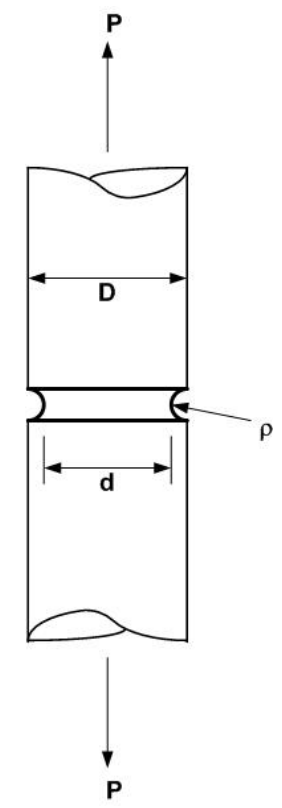

Figure 2-2: Geometry of a round bar with grooved notch typically used in stress concentration calculations.

The stress concentration factor, $\mathrm{K}_{\mathrm{T}}$, is defined as the ratio of the maximum stress at a notch root to the nominal stress. The nominal stress can either be defined as the load divided by the area at the notch root or the area of the main member cross section, but typically the root area is used to calculate the nominal stress [8]. Calculation of the theoretical stress concentration depends solely on the geometry of the specimen and can only be applied when there is no plastic deformation. The general equation for calculating $\mathrm{K}_{\mathrm{T}}$ is shown as Equation 2-1, while a more specific equation for calculating $\mathrm{K}_{\mathrm{T}}$ for the notch geometry is shown in Figure 2-2 can be seen in Equation 2-2 [6].

\section{Equation 2-1}

$$
\mathrm{K}_{\mathrm{T}}=\frac{\sigma_{\max }}{\sigma_{\text {nom. }}}
$$




\section{Equation 2-2}

$$
\mathrm{K}_{\mathrm{T}}=1+\left[\frac{\left(\mathrm{K}_{\mathrm{te}}-1\right)^{2} \cdot\left(\mathrm{K}_{\mathrm{th}}-1\right)^{2}}{\left(\mathrm{~K}_{\mathrm{te}}-1\right)^{2}+\left(\mathrm{K}_{\mathrm{th}}-1\right)^{2}}\right]^{0.5}
$$

where:

$\mathrm{K}_{\mathrm{th}}=\frac{\left[\frac{\mathrm{r}}{\rho} \cdot\left(1+\frac{\mathrm{r}_{\mathrm{d}}}{\rho}\right)^{0.5}+(0.5+\mathrm{v}) \cdot\left(\frac{\mathrm{r}_{\mathrm{d}}}{\rho}\right)+(1+\mathrm{v}) \cdot\left[\left(1+\frac{\mathrm{r}_{\mathrm{d}}}{\rho}\right)^{0.5}+1\right]\right]}{\mathrm{N}_{1}}$

$\mathrm{N}_{1}=\left[\left(\frac{\mathrm{r}_{\mathrm{d}}}{\rho}\right)+2 \cdot \mathrm{v} \cdot\left(1+\frac{\mathrm{r}_{\mathrm{d}}}{\rho}\right)^{0.5}+2\right]$

$\mathrm{K}_{\mathrm{te}}=1+2 \cdot\left(\frac{\mathrm{c}}{\rho}\right)^{0.5}$

$\mathrm{C}=\frac{(\mathrm{D}-\mathrm{d})}{2}$

$r_{d}=\frac{d}{2}$

$\rho=$ root radius

$\mathrm{v}=$ Poissons ratio

The notch sensitivity produced by threads on fasteners can also have an effect on the maximum load that a fastener or notched member can withstand. Notch sensitivity is affected by both the geometry and the mechanical properties of the specimen. Notch sensitivity, q, is defined as follows in Equation 2-3 [8]: 


\section{Equation 2-3}

$$
\mathrm{q}=\frac{\left(\mathrm{K}_{\mathrm{e}}-1\right)}{\left(\mathrm{K}_{\mathrm{T}}-1\right)}
$$

where $\mathrm{K}_{\mathrm{e}}$ is defined as the ratio of the fracture load of a smooth regular rod to that of a notched rod having the notch diameter equal to the diameter of the regular rod. Empirical data has been collected for the determination of notch sensitivity of quenched and tempered steels with various strength levels [9]. Equation 2-4 utilizes the empirical data for calculating the sensitivity of metals:

\section{Equation 2-4}

$$
\mathrm{q}=\frac{1}{1+\frac{\alpha}{\rho}}
$$

where $\rho$ is the notch radius and $\alpha$ is an empirical constant dependent on strength level.

\section{2: Hydrogen Induced Cracking (HIC)}

\subsection{1: Mechanisms and Theories}

Hydrogen induced cracking and the mechanisms involved with hydrogen induced cracking have been studied and theorized for a very long time, with a peak interest in the 1950's and 60’s. Although there are too many publications on the subject of hydrogen effects on metals to count, no single theory for the mechanism has been fully established. Several of the proposed and accepted mechanisms for the deleterious effect of hydrogen on steel are mentioned by Timmins in his book "Solutions to Hydrogen Attack in Steel” [10]. One of the earliest theories was published by Zapffe and Sims, who believed that a build-up of hydrogen gas created a pressure concentration that lowers the fracture strength. Another theory mentioned by Timmins, and reviewed later, is the decohesion concept postulated by Troiano. Other theories cited by Timmins include the idea of a 
lowered surface energy required to propagate a crack (Petch), the possibility of formation of hydrides or other hydrogen phases, that lower the fracture strength of the steel (Westlake), and finally a theory first postulated by Beachem who found evidence that hydrogen made dislocation movement easier, so that extreme localized plasticity causes HE. The theories postulated by Troiano, Beachem and Petch will be reviewed here, since they are most applicable to the work performed throughout this project.

\subsubsection{A: Troiano's Decohesion Theory}

Around 1960 Troiano [2] and Steigerwald, collaborating with Troiano [11], presented their theory for the mechanism of hydrogen induced cracking in steel and other body centered cubic metals. They were also able to relate and test experimentally some of their ideas to other elements such as carbon, nitrogen and oxygen that reside at interstitial sites in metals. The main criterion for HIC is a localized hydrogen concentration at dislocation arrays just below a notch root, which was proven to be the site of maximum triaxial stress. The position of the maximum stress moves inward toward the center of the specimen as the notch severity, or stress concentration, is lowered. The hydrogen then acts to weaken the cohesive bonds between the lattice planes, resulting in a crack or void. Kitajima [12] also showed through modeling that decohesion is indeed an adaquate mechanism for the propagation of a sharp crack.

It was shown that the delayed failure of metals by HIC can be described in terms of four stages or levels. First there exists an upper critical stress, which is the failure strength of the material without the existence of hydrogen. There also exists a lower critical stress, where hydrogen essentially has no effect on lowering the strength of the metal, since the hydrogen cannot sufficiently segregate to the stress gradient area. This 
stress is often termed the threshold stress. The other stages of HIC are the incubation or nucleation of the cracks and the propagation of the crack to failure. The fracture rate of the delayed failure, related to both nucleation and propagation of the crack, is a function of the stress concentration, which acts as the chemical potential gradient for the diffusion of hydrogen atoms. The temperature and strain rate also affect the fracture times, again due to the ability of the hydrogen to accumulate significantly at the notch root area. High strain rates and low temperatures do not allow for adequate diffusion, therefore fracture is delayed for longer periods.

Troiano also proved that increases in the hydrogen content, notch severity and strength level were critical in controlling the susceptibility of metals to HIC by lowering the threshold stress, nucleation rate of cracks and, eventually, failure times. There appears to be a minimum gross hydrogen concentration of around 5 ppm for adequate hydrogen to be supplied to the maximum triaxial stress location, and if more hydrogen is present it is easier for the hydrogen to accumulate. Critical hydrogen concentration is a function of the interaction energy between the interstitial hydrogen atoms and the stress field about the fracture nucleus. As the notch severity increases, the interaction energy remains the same, but the notch allows for the critical stresses to be reached at lower applied loads. Effects of the strength level of the steel on HE are also not related to the interaction energy unless the critical hydrogen content must be changed, yet the threshold stress will be lowered at higher strength levels. This can be explained by the lower yield strength in lower strength steels, which initiates plastic flow and decreases the maximum value of stress that can be attained. This means that the lower critical stress must be higher to obtain the critical hydrogen concentration. The lower yield strength also 
decreases the stress concentration so a higher load is required to generate enough hydrogen at the maximum triaxiality.

On the atomistic level, Troiano suggested that the filling of the metal d-bands with excess electrons from hydrogen may be responsible for the loss of cohesive strength between the atoms. The filling of the d-band may result in increased repulsive forces. It was believed that by alloying with elements that lowered d-band electron concentrations, HE could be reduced. This was substantiated through an experiment showing that nickel alloyed with chromium or iron, which lowers the d-band electron concentration, did indeed show a decrease in HE susceptibility.

\subsubsection{B: Petch's Surface Energy Reduction Theory}

Petch [1] used the Griffth and dislocation theories to explain the loss of strength in iron based materials due to hydrogen by a lowering of the surface energy required to propagate a crack. Iron fracture surfaces can act as excellent chemisorbers of hydrogen and the hydrogen can diffuse to crack tips, where stress concentrations are high, very quickly at temperatures near room temperature. Through calculations, Petch showed that the surface energy of iron is reduced drastically even when very small amounts of hydrogen are present. This reduction in surface energy was related to a reduction in fracture strength for hydrogen charged samples. It was also observed that the charged and uncharged samples both followed a Hall-Petch relationship with respect to grain size, but the trend for the charged samples showed a decrease in slope and intercept. The decrease in slope is attributed to the lowering of the surface energy when hydrogen is present, while the change in intercept is related to the loss in ductility due to the lowering of the slope value. 


\subsubsection{C: Beachem's Model for Hydrogen-Assisted Cracking}

Beachem [3], in the 70's, proposed a model that hydrogen actually can act to promote local plastic deformation, which is essentially opposite to a theory of embrittlement. Overall, it was believed that hydrogen served to enhance whichever fracture mode, intergranular (IG), microvoid coalescence (MVC), cleavage, and/or quasicleavage (QC) is normally activated. The theory was supported by the observation of microvoid coalescence fracture in continuously hydrogen charged steels. This suggested that the hydrogen made dislocation multiplication and motion easier at lower stresses, and thus acts to create localized plastic deformation at these lower stresses. Hydrogen induced cracking is typically associated with a lack of ductility and not increased plasticity. This was explained by Beachem by the fact that local plasticity due to hydrogen charging was constrained by the uncharged non-ductile material surrounding the plastic zone. This is similar to the phenomenon for notched tension specimens. The expansion of the local plastic zone is limited by the diffusion of the hydrogen, and therefore the crack will propagate by means of a brittle fracture through the uncharged area. The result of this behavior is a decreased reduction in area of smooth specimens when hydrogen is present.

The effect of hydrogen on steels was stated to depend upon chemistry of the steel, heat treatment of the steel, the stress intensity at the crack root and the amount of hydrogen present. A plot of stress intensity as a function of hydrogen content was created to visualize the theory given a steel and heat treatment, showing the differences in the fracture modes that would be present. A schematic of this plot is shown in Figure 23. 


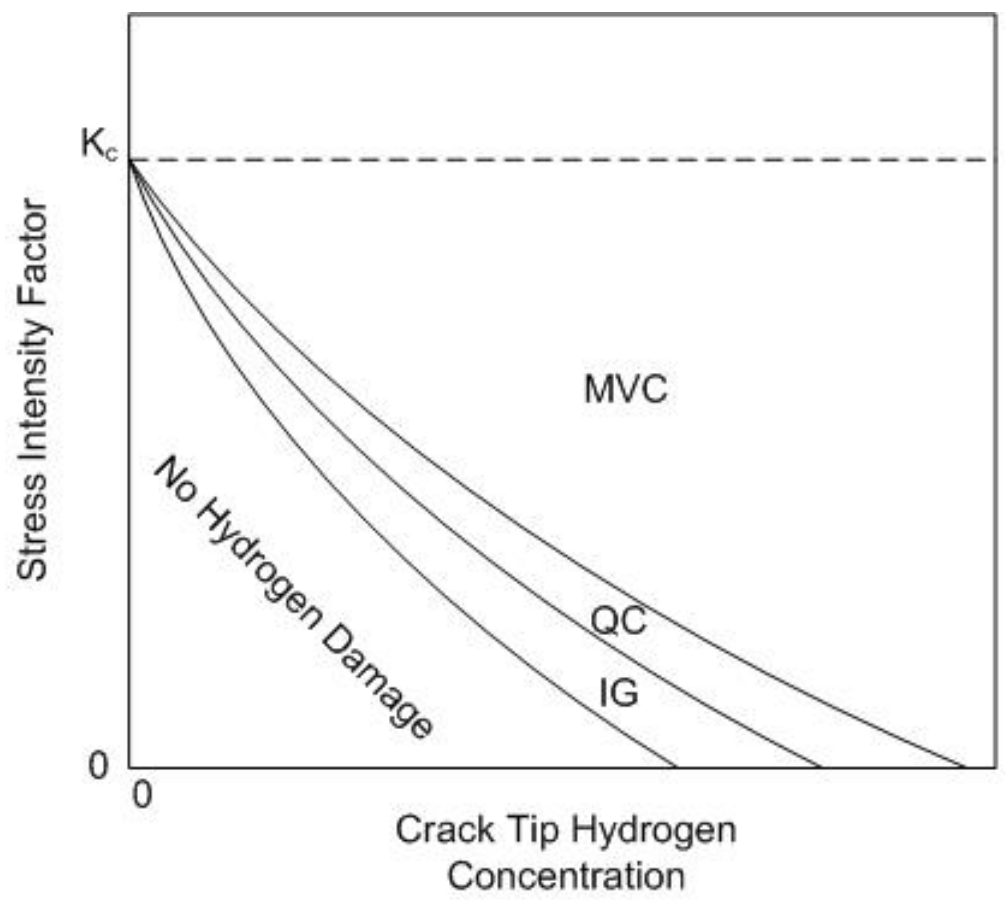

Figure 2-3. Schmatic representation of the fracture modes possible in steel when hydrogen cracking is involved [3].

Support of the localized plasticity theory due to hydrogen in steel was observed by Nagumo [13] and Kinaev et al. [14]. Experiments by Kinaev were conducted by measuring crack tip opening displacement and strain fields in fatigue pre-cracked fracture mechanics specimens that were tested in a vacuum, hydrogen gas atmosphere or water vapor atmosphere. The strain gradient in the samples tested in the hydrogen atmospheres and water vapor showed a significant increase in plasticity compared to that of the samples tested in vacuum. The crack tip opening displacement of the specimens tested in a low hydrogen pressure was similar to that of the specimens in air, even though the plastic zone and local strains were higher. At higher hydrogen gas pressure and in water 
vapor the plastic zone and crack tip opening displacement were significantly larger than in vacuum. At longer time intervals the opening of the crack tip increased for the specimen at the high hydrogen pressure, indicating plasticity through creep. Kinaev et al.'s work can be used to verify that hydrogen does induce localized plasticity at crack openings and there may be either a critical hydrogen concentration required to initiate the plasticity or a time dependence to activate the plasticity.

\subsection{2: Test methods for Hydrogen Induced Cracking Resistance}

Several test methods have been devised for quantifying the effects that hydrogen has on materials when they are stressed. Testing methods have also been specifically developed to test the strengths of fasteners with and without hydrogen present. ASTM standard F 606 gives a detailed description of the proper testing of the mechanical properties of threaded fasteners, which includes: hardness measurements, standard tension testing, wedge tension testing, and wedge testing for embrittlement [15]. The embrittlement wedge testing is designed to test coated fasteners that may have residual hydrogen trapped within the fastener due to the coating. During the wedge test a fastener is loaded in tension to a minimum of $75 \%$ the ultimate strength with a wedge of a specific angle placed underneath the head of the bolt for a minimum of 48 hours. Following the testing period, the fastener will pass if it shows no sign of failure due to embrittlement. Other test methods for hydrogen embrittlement include a DaimlerChrysler (DCX) torque test similar to ASTM F 606, a GM bend test, the slow strain rate tension test (SSRT) and the rising step load test (RSL). The RSL testing can be performed in either tension or bending. 
McCarthy et. al. [16] performed a study to compare three HE testing methods; the DCX torque test, GM bend test and RSL bend test. This comparison was performed specifically to test for the effect of residual hydrogen on steel fasteners. The study showed that the RSL bend test produced the most definitive and reproducible results, but the application of the bend was not appropriate and the test should be carried out in tension. The torque down test was found to be the least accurate in predicting the HE behavior of the fasteners. The draw backs of the RSL test are that only one sample can be tested at a time, whereas the other tests can be set up to run several samples, and the RSL equipment is more expensive. The authors deemed that the RSL tension test would be a good test to identify the threshold stress that a fastener could withstand.

Raymond [17], the designer of the RSL testing procedure, also has compared RSL testing to other methods. Raymond has identified problems with the wedge embrittlement test defined in ASTM F 606. These problems are: lack of reproducibility, typically due to machining tolerances, often inadequate testing times, and a possible danger of projectiles during failures. The article also points out problems with the SSRT, such as the lack of correlation between testing and service behaviors and the lack of addressing the notch sensitive behavior of fasteners during testing. The benefits of the RSL testing that were not mentioned previously are its ability to easily test in tension or bending and the fact that a wide spectrum of samples, including actual bolts and precracked samples, can be tested. It should be noted that the SSRT and the RSL tension tests are similar in that the load is increased by displacement over an extended time period in order for the kinetics of HE to take effect, but due to the recent advent of the 
RSL most of the research on quantifying HE threshold stresses has been performed using other methods.

RSL testing can be used to quantify the HE resistance of steels with different heat treatments or compositions. The test can also be utilized to measure the effect that residual hydrogen absorbed during processing has on the strength of a given steel, or the effect the hydrogen has when it is absorbed from a specific environment. The testing during this project was performed under the same conditions for all samples, but the chemistry and heat treatments of the steels were varied to see the effect on the threshold stress.

ASTM F 1624 defines the procedure for testing HE susceptibility utilizing a rising step load. Initially a continuously increasing load is applied at a relatively rapid rate to the sample, in ambient air, until the specimen fails. This test is performed on two or three samples and the failure load of the samples is averaged to obtain the critical load required to rupture the specimen, $P_{\mathrm{c}}$ [18]. The threshold load, $P_{\text {th }}$, is then measured by loading a hydrogen charged sample at selected fractions of $P_{\mathrm{c}}$ for a given time until failure occurs. The time at each increment is extended for each protocol until the time delayed effect is not noticeable and the fracture load for a given protocol, $P_{\mathrm{i}}$, does not change when the holding time is increased, indicating that diffusion rates of hydrogen are not controlling. This value is defined as the threshold load, $P_{\text {th }}$. Figure 2.4 shows schematically the testing protocol used in RSL testing to determine the threshold load of a sample of given geometry, heat treatment and composition. 
Load Vs Time

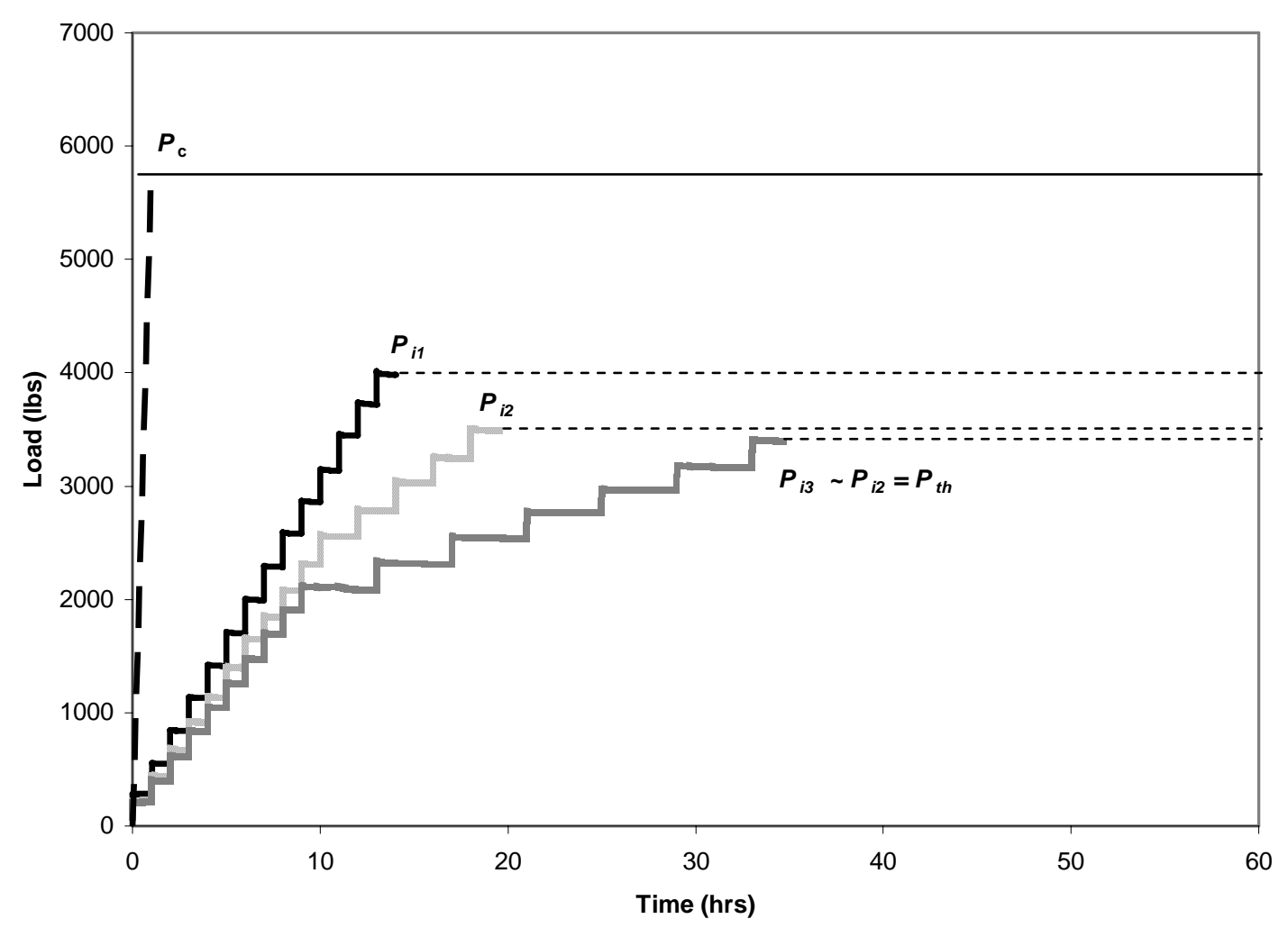

Figure 2-4. Schematic representation of the rising step load protocol used in finding the threshold load of the sample.

\section{3: Steel Microstructures and Effects of Microstructures on HIC}

\subsection{1: Dual Phase Structure}

Dual phase steels were developed with applications in the auto industry where low weight and low cost of easily formable steel are required. Typically, dual phase steels have low carbon contents of around 0.1 weight percent and the microstructure consists of a ferrite matrix with islands of hard un-tempered martensite. The generation of a dual phase structure is evolved by annealing the steel at an inter-critical temperature 
within the ferrite plus austenite phase region, then quenching so that the austenite transforms to martensite. A phase diagram of steel, showing the intercritical region, can be observed in Figure 2-5. It has been shown that the strength of the dual phase steels can be increased by modifying the heat treatment to produce more martensite or by changing the morphology of the martensite $[19,20]$.

\section{Iron-Carbon Phase Diagram}

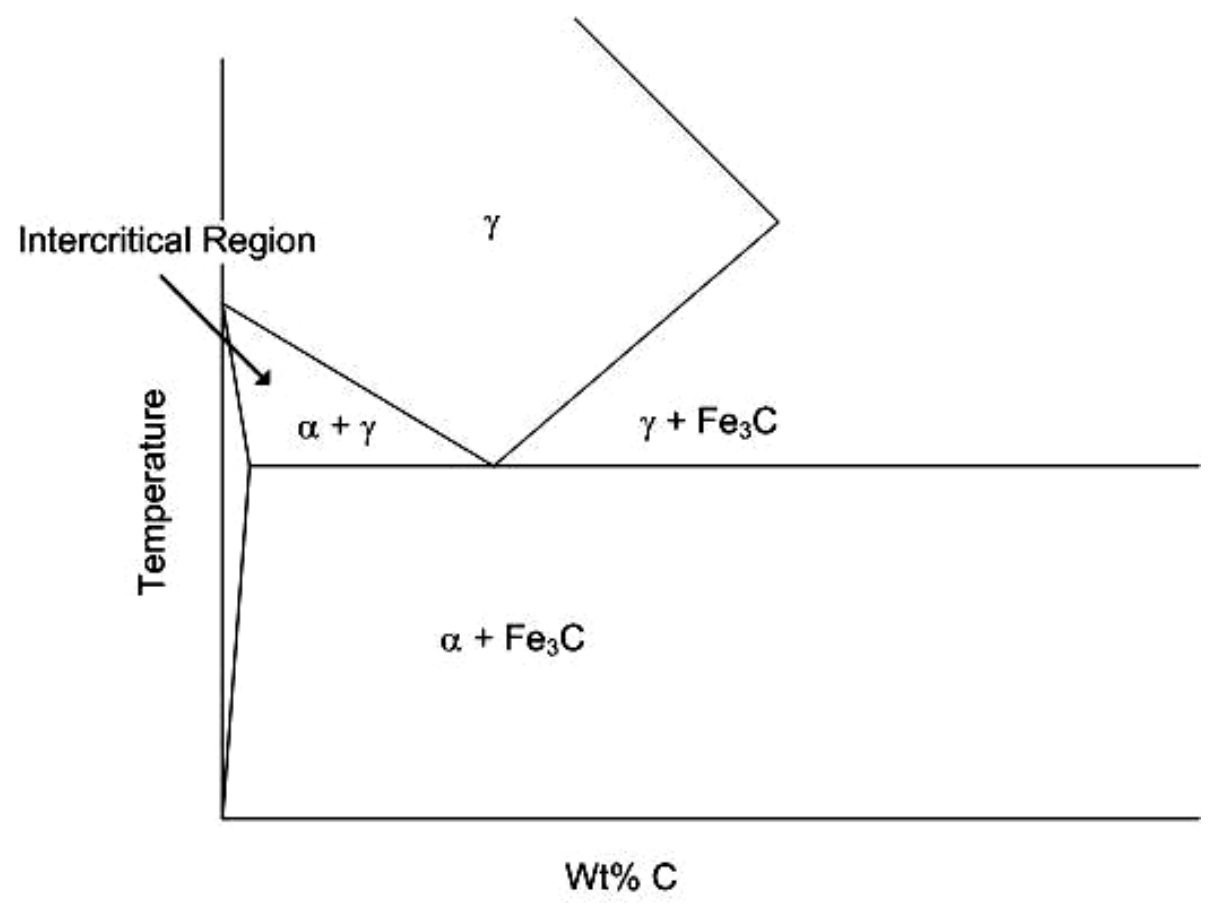

Figure 2-5: Schematic iron-carbon phase diagram showing the intercritical ferrite+austenite region used in dual phase heat treatments. 
Bayram et al. [19] defined three heat treatments for generating dual phase structures and then compared the tensile, notch and fracture properties of the steels. The three heat treatments included: an intercritical anneal (IA) by heating into the two phase region then quenching into oil, a step anneal (SA) by austenitizing followed by quenching into oil, then a re-heat to the two phase region followed by a final quench, and a continuous anneal (CA) where the steel was austenitzed, slow cooled into the two phase region, then quenched. The three heat treatments can be visualized schematically in Figure 2-6. All microstructures consisted of ferrite and martensite with the CA and IA steels having similar structures, but the SA heat treatment consisted of smaller fiber-like martensite and ferrite grains with higher dislocation densities due to the higher surface area to volume ratio of the austenite grains as they transformed to martensite. Also, the ferrite grains in the SA specimens had a sub-grain structure due to the recovery, recrystallization and grain growth occurring during the transformation from martensite in the two phase region. The mechanical and notch testing of the samples showed that the IA sample had the highest yield strength followed by the SA specimens. The SA heat treatment resulted in significantly higher elongation and fracture toughness when compared to that of the other two heat treatments. 

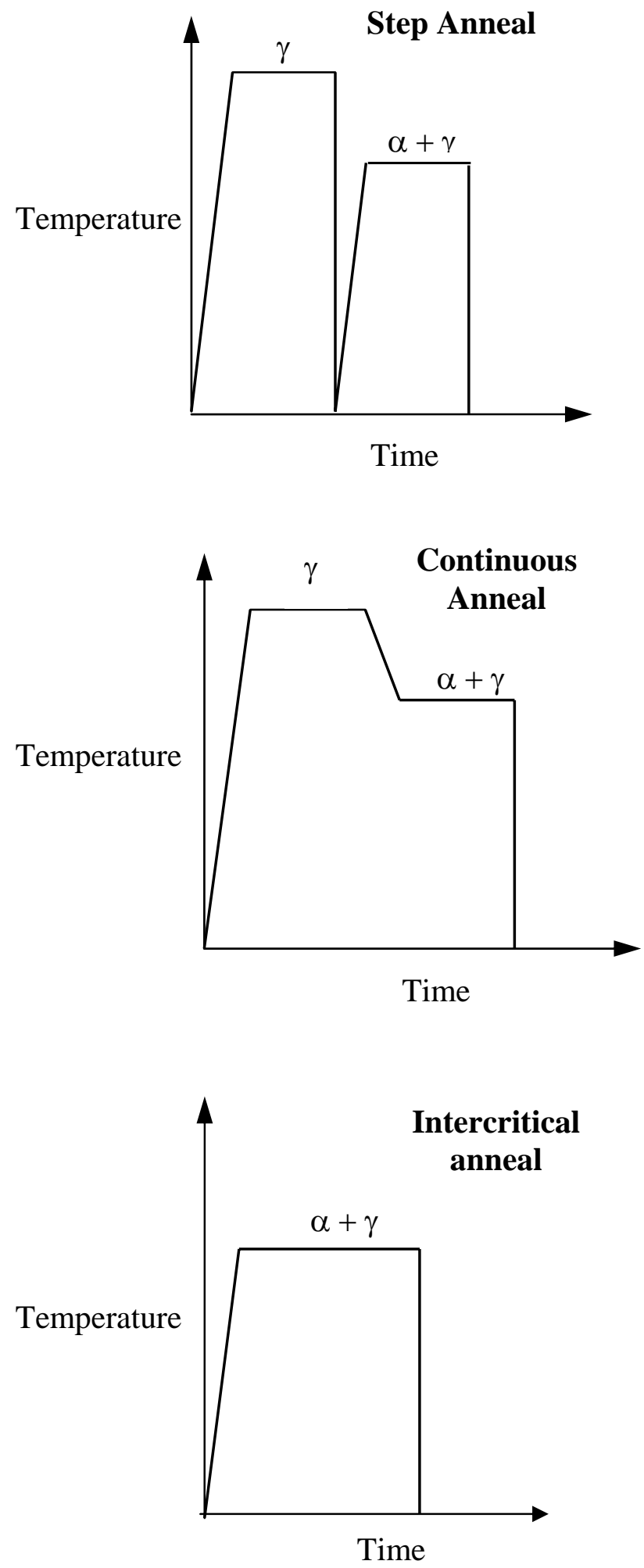

Figure 2-6: Various heat treatment schedules used to produce a dual phase steel microstructure. 
Ahmad et al. [20] performed an intercritical anneal on a medium carbon steel at various temperatures ranging from the eutectoid temperature to a temperature just above the two phase region, in the gamma region. This produced a dual phase structure with martensite volume fractions ranging from about $30 \%$ to $100 \%$. The strength of the steel increased as the martensite content increased, but the elongation decreased. The fracture process in the steels was observed to be a micro-void process with nucleation at the ferrite-martensite interface when there was a lower percentage of martensite present, but changed to a micro-cracking brittle mechanism when the martensite content was increased.

Grochowski [4], in the previous work related to this research project, performed RSL testing on AISI 4037, heat treated by the continuous annealing method to produce a dual phase structure. The high carbon content and high annealing temperature produced a structure with a martensite matrix and around $12 \%$ ferrite. The samples were then heated and held at various temperatures to temper the martensite and obtain a range of hardness values. During the RSL testing the samples showed notch sensitivity when tested in air, due possibly to a micro-cracking behavior at the ferrite-martensite interfaces. The RSL samples tested in a salt water solution showed a drop off in threshold stress as the hardness of the samples increased, as observed in most other steels. In another study by Kerr et al. [21], it was observed that the critical stress intensity for stress corrosion cracking of a dual phase, ferrite matrix with martensite islands, was higher than that of a fully martensitic structure.

The morphology changes due to a step anneal process may change the notch sensitivity and resistance to HIC of dual phase ferrite plus martensite steels. Specimens 
of 4037 steel were heat treated by the step anneal and temper process to generate samples with varying hardness. These samples were subsequently tested for resistance to HIC using the RSL testing method.

\subsection{2: Martensite Versus Bainite}

The martensite reaction involves heating steel into the face centered cubic (fcc) austenite phase field followed by quenching into an aggressive cooling medium so that diffusion does not take place during the transformation. The martensite transformation is a shear transformation, wherein the carbon atoms in the fcc phase are trapped at the interstitial sites when the material attempts to transform to the low temperature body centered cubic (bcc) structure. The resulting crystal structure is body centered tetragonal (bct), with carbon atoms trapped at interstitial sites. The strengthening mechanism of the martensite is due to the solid solution hardening as well as the dislocation strain hardening from the transformation. Quenched martensite has a very high hardness; therefore the material is typically tempered at a moderate temperature. The initial stage of tempering involves relieving of the residual stresses accumulated during quenching. Then the carbon diffuses out of the body centered structure and forms carbides. With the diffusion of the carbon to form carbides, the matrix microstructure returns to that of bcc ferrite.

The bainitic reaction produces a structure that is similar to that of martensite, but the transformation is a diffusional transformation that typically occurs isothermally at temperatures below the pearlite temperatures and above the martensite start temperature. The isothermal treatment following austenitizing is often referred to as austempering. Improved toughness and ductility of bainitic structures at a given hardness, when 
compared to martensitic, make bainitic steels more attractive in certain applications. The reduction in area of a tensile sample for bainitic steels may be nearly double that for tempered martensite steels [22]. There is also less distortion when performing an isothermal heat treatment due to the differences in transformation behavior and less thermal distortion. Typical applications for bainitic steels are fasteners, clips, springs and lawn mower blades.

Bainite can exist as either upper or lower bainite, where upper bainite forms at the higher temperatures and lower at low temperatures; for example, a plain carbon 1035 steel will form lower bainite at temperatures between approximately $450-550^{\circ} \mathrm{C}$ and upper bainite between the temperatures of about $550-580^{\circ} \mathrm{C}$. At the high bainitic reaction temperatures the kinetics are rapid enough to allow for carbon to completely diffuse from the supersaturated ferrite laths that form initially, resulting in carbide platelets between ferrite [23]. At lower temperatures, the slower diffusion rate of carbon provides enough time for carbide particles to nucleate within the ferrite laths as well as between laths [23]. A schematic representation can be seen in Figure 2-7. 


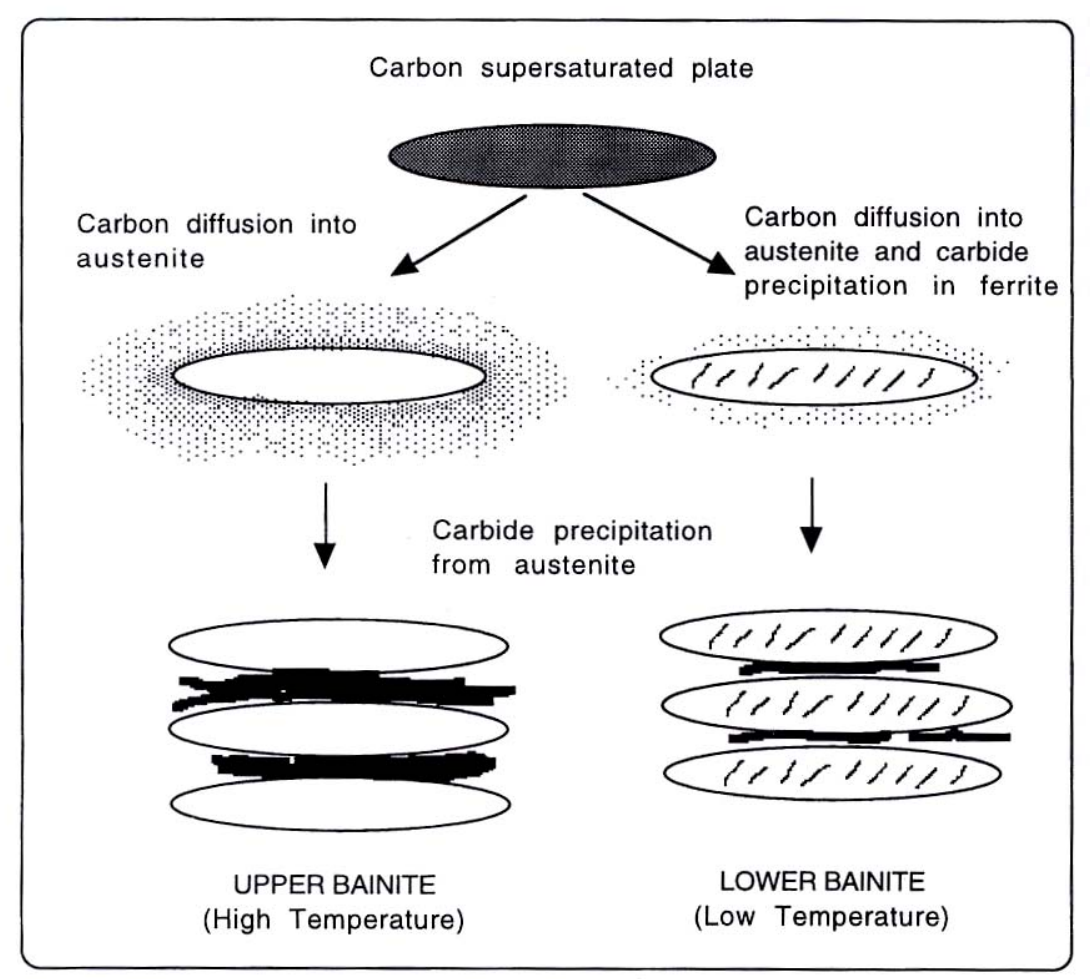

Figure 2-7: Schematic representation of the formation of upper and lower bainite [23].

Carbon concentration of the steel plays an important role in the transformation behavior of martensite and bainite. As the carbon concentration is increased, the martensite and bainite start temperatures are lowered. Due to the thermodynamics and kinetics of the bainite reaction, carbon concentration also determines whether upper or lower bainite will form at a given isothermal temperature. Figure 2.8 is an illustration of the effect of carbon on the bainite start $\left(B_{s}\right)$ and martensite start $\left(M_{s}\right)$ temperatures. If the carbon concentration is high then the kinetics are slower and only lower bainite will form. If the carbon concentration is low, then there is an increased diffusion rate and only upper bainite can exist. There are also carbon concentrations and temperatures that 
may produce a mixed upper and lower bainite structure or mixed lower bainite and martensite structure [23].

Upper \& Lower Bainite

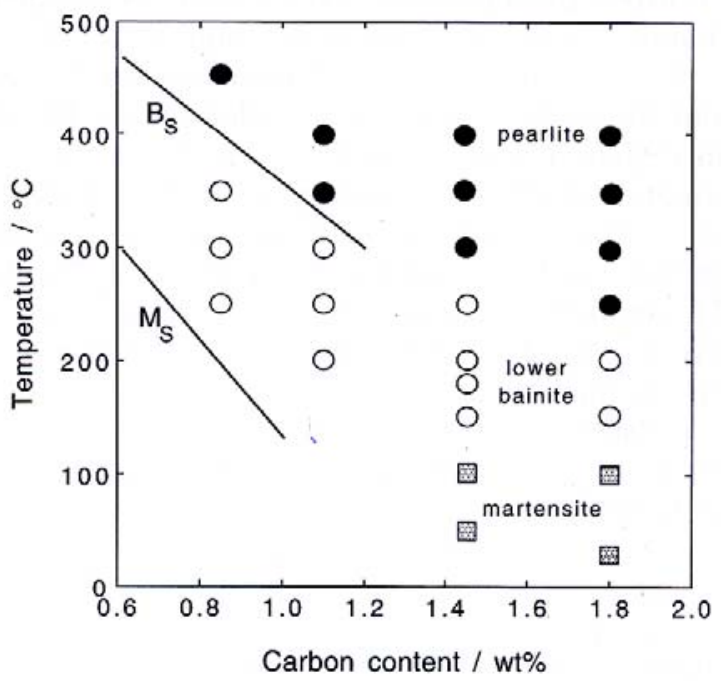

Figure 2-8: Bainite and martensite start temperatures as a function of carbon concentration [23]

The HIC behavior of steels containing fully tempered martensite structures has been studied extensively, and the resistance to HIC is often related to the hardness of the tempered steel [24, 25, 26, 27]. As the hardness, or yield strength, is increased the resistance to HIC decreases. Many investigators believe that the strength level is not the determining factor in hydrogen embrittlement and that microstructural features may be more important in assessing the resistance to HIC [24, 28].

McCarthy et al. [24] performed RSL studies on 1022 and 10B21 steels that were hardened through quench and temper treatments. It was discovered that small changes in 
tempering temperature resulted in drastic changes in the microstructure, which, in turn, changed the susceptibility to hydrogen assisted cracking. A decreased susceptibility was attributed to a morphology change of the carbide particles from long plates to small spheres. It was determined that tempering temperature is a better indicator of resistance to HIC than hardness. Quadrini [27] hypothesized that more thermodynamically stable microstructures, i.e. higher tempering temperatures, are less susceptible to hydrogen induced cracking. Also, it was mentioned that larger carbides, again associated with higher tempering temperatures, require higher threshold stresses to nucleate cracks or micro-voids, resulting in increased resistance to HIC.

Thompson et. al. [28] presented work on 4140 steel, comparing the susceptibility of hydrogen charged bend specimens having both quenched and tempered and bainitic structures. The crack initiation loads dropped significantly more for the quenched and tempered samples that were hydrogen charged when compared to the bainitic specimens. It should be noted though that the initiation load was lower in air for the bainitic sample. The fracture mode was the same for both martensitic and bainitic samples, consisting of an intergranular mode at the notch root and micro-void coalescence deeper into the sample. The percentage of area consisting of intergranular fracture was much higher for the quenched and tempered than the bainite specimens.

Kerr et al. [21] studied the critical stress intensity for stress corrosion cracking of specimens containing either a martensitic, bainitic or dual phase ferrite/martensite structure. It was found that the highly tempered martensitic structure produced the most resistance, followed by the bainitic and dual phase structures, with the most susceptible structure being that of untempered quenched martensite. Two studies on hydrogen 
induced fatigue crack growth showed contradictory results on the effect of bainitic structures compared to tempered martensite structures at similar yield strengths $[29,30]$. In one study there was no pronounced difference in the crack growth rate as a function of stress intensity for samples of varying microstructure [29]. In the other study, highly tempered martensite showed the slowest crack growth, followed by the bainitic structures, with the low temperature tempered martensite structures having the quickest growth rates [30]. Bainitic structures of either lower or upper bainite showed a similar growth rate, which was attributed to cracking along inter-lath carbide particles.

The morphology of the carbides and ferrite in bainite may reduce the susceptibility of steel to hydrogen assisted cracking when compared to tempered martensite. In this work several different alloys were heat treated to produce specimens of the two different microstructures (bainite and tempered martensite) at similar hardness levels. These specimens were then tested using the RSL method to determine the threshold fracture stresses and to determine if variations in the microstructure do indeed affect the resistance to HIC.

\subsection{3: Alloying Element and Carbon Content Effects on HIC}

Fastener grade steels are typically alloyed to increase the hardenability of the steel, ensuring the material is through hardened upon quenching. Carbon may be added to obtain a steel if high strength is required, since increases in carbon content increase the strength attainable through quenching and tempering. Alloying elements present in steel may play a role in several different interactions with hydrogen, such as local hydrogen concentrations, trapping and diffusion, changes in matrix or interface cohesion, dislocation mobility and resistance to crack nucleation, propagation or crack arrest [31]. 
Typical fastener grade steels are 1022, 10B21, 1038, 1065, 1095, 1541, 4037 and 4140, and contain alloying elements such as carbon, boron, manganese, molybdenum and chromium.

The amount of carbon in a given alloy controls not only the attainable hardness of a given steel but also the amount and type of carbides that are present following heat treatment. The weight fraction of carbide in plain carbon steel can be estimated using the lever rule. Carbide content and morphology may adjust the trapping behavior, which can contribute to hydrogen assisted cracking. Although effects of alloying elements on HIC behavior are typically not studied systematically, some data for various elements exist. Bernstein et. al. [31] has summarized studies of alloying effects on hydrogen assisted cracking. Manganese is one element that is believed to cause increased susceptibility to hydrogen cracking. Elements that may be beneficial are silicon, vanadium, niobium, palladium and boron. Two studies have shown that boron has little effect on the susceptibility of steel to HIC but results may be more reproducible [24, 32]. Typical hardening agents, chromium, nickel and molybdenum, show mixed results [31].

The trapping behavior of steels can cause pronounced changes in the diffusion of hydrogen through the steel. Traps are usually classified into two categories, reversible and irreversible. Reversible traps are low energy traps, where hydrogen is capable of being released at room temperature. High energy trap locations, from which hydrogen cannot be removed, are classified as irreversible traps. Typical reversible traps are dislocations, grain boundaries and precipitate interfaces, while irreversible traps are usually carbides or oxide and sulfide inclusions. The hydrogen diffusion rate in bcc iron is around $7.0 \times 10^{-9} \mathrm{~m}^{2} \mathrm{~s}^{-1}$, but is reduced to about $2.0 \times 10^{-11} \mathrm{~m}^{2} \mathrm{~s}^{-1}$ when the structure is 
quenched martensite [26]. The lower effective diffusion rate for quenched martensite is due to the dislocations that act as reversible traps. During tempering the density of dislocations is reduced and the diffusion rate of hydrogen initially increases, then decreases again as irreversible trapping carbides are precipitated [26, 30].

Several different alloys were quenched and tempered to produce tempered martensite at similar hardness levels and were then tested in tension to measure the threshold stress of each alloy at a given hardness. Also, three plain carbon steels were quenched and temper heat treated to similar hardness levels, in an effort to determine the effect of carbon content on resistance to HIC.

\section{4: Fractography}

Fracture surfaces can often provide investigators with a map of the possible failure mechanisms. There are typically four fracture modes: micro-void coalescence (MVC), cleavage (C), quasi-cleavage (QC) and intergranular (IG). It is possible and even likely that two or more of the fracture modes occur during failure. Micro-void coalescence failure is usually associated with a plastic failure mechanism, while the others are typically more of a brittle mechanism. Properties associated with fracture surfaces, such as dimple size of voids in MVC or facet size of cleavage plates can be helpful in identifying possible fracture-microstructure relationships. Figure 2-9 displays schematically four modes of fracture. Fracture surfaces of all batches of specimens tested under RSL conditions were observed to help identify possible fracture modes in hydrogen charged and uncharged specimens. 

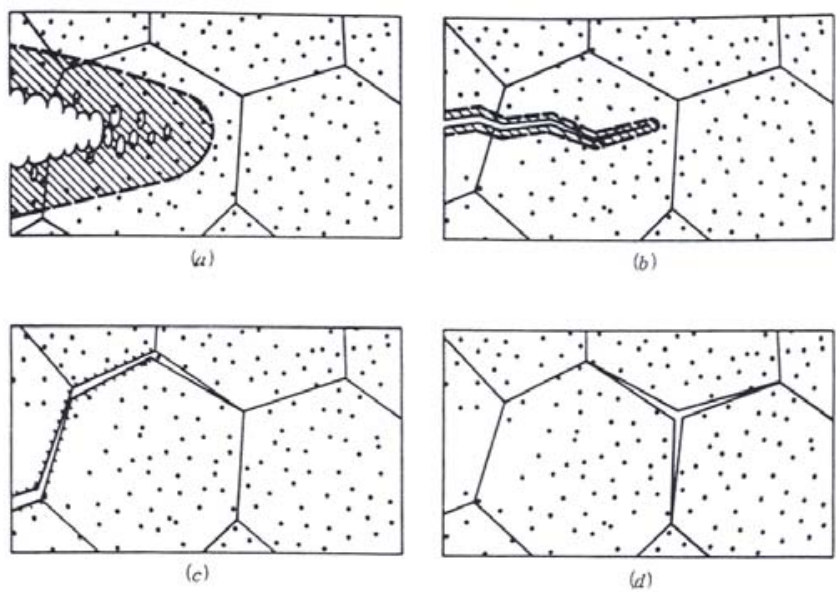

Figure 2-9: Illustration of fracture modes. (a) micro-void coalescence, (b) quasi-cleavage, (c) low stress intensity intergranular, (d) high stress intensity intergranular [3].

\section{5: Ferrite Crystallite Size}

The mean free path between carbides on the boundaries of ferrite can be estimated by measuring the peak breadth of the ferrite diffraction peak. The x-ray diffraction peak breadth is a function of the crystallite size, strain in the sample and broadening due to the instrument. The macro-strain from polishing can be reduced by special polishing techniques and broadening due to the instrument can be adjusted for by measuring the width of peaks in annealed iron powder samples. Separation of the micro-strain broadening from crystallite broadening can be performed by plotting $\mathrm{B}(\cos (\theta))$ as a function of $\sin (\theta)$. This leaves an easy calculation for the crystallite size, the Sherrer's equation, shown as Equation 2-5:

\section{Equation 2-5}

$$
\mathrm{t}=0.9 \frac{\lambda}{\mathrm{B} \cos \theta}
$$


where $t$ is the crystallite size, $\lambda$ is the wavelength of the $x$-rays, $\theta$ is the peak position angle and B is the full width at half maximum (FWHM) of the peak [33]. This equation can be applied to the FWHM after instrument broadening, Gaussian and Lorentz corrections are taken into account.

Knowing the ferrite crystallite size can help to identify the spacing between carbides in steel and can be used to relate the strength of the steel to microstructural features that may change the resistance of a given steel to HAC. The crystallite size was estimated for all 4140, 1065 and 1095 specimens by x-ray diffraction. 


\section{Chapter 3: Experimental Procedure}

\section{1: Notched Tension Sample Preparation and Heat Treatment}

\subsection{1: Sample Preparation}

Coiled steel wire of the selected alloys, with a diameter of 3/8”, was acquired from Charter Steel, Inc. The compositions of the steel wires obtained for testing are shown below in Table 3-1. The wire was then sent to Era Wire, Inc, where it was run through a straightening machine and cut to 4" pieces. The 4" pieces were shipped to Jantom Precision, Inc for the initial machining. The initial machining consisted of a rough grinding of the entire sample followed by roll threading the ends of the samples.

After the initial machining was completed, the samples were either sent back to Michigan Tech for heat treating or the samples were heat treated through Jantom Precision, Inc.

Once the samples were heat treated, Jantom Precision, Inc. used a centerless grinder to form the gauge length and generate the precision notch. The sample geometry conforms to a modified ASTM F519 standard [34], of which a detailed, un-scaled, drawing can be seen in Figure 3-1.

\begin{tabular}{|c|c|c|c|c|c|c|c|c|c|c|}
\hline \multirow{2}{*}{ Grade } & \multicolumn{10}{|c|}{ Weight Percent } \\
\cline { 2 - 12 } & $\mathbf{C}$ & $\mathbf{S i}$ & $\mathbf{M n}$ & $\mathbf{S}$ & $\mathbf{P}$ & $\mathbf{C r}$ & $\mathbf{N i}$ & $\mathbf{M o}$ & $\mathbf{C u}$ & $\mathbf{B}$ \\
\hline $\mathbf{1 0 B 2 1}$ & 0.23 & 0.27 & 0.96 & 0.01 & 0.01 & 0.09 & 0.05 & 0.01 & 0.09 & 0.0028 \\
\hline $\mathbf{1 0 3 8}$ & 0.37 & 0.24 & 0.73 & 0.01 & 0.011 & 0.08 & 0.06 & 0.02 & 0.1 & - \\
\hline $\mathbf{1 0 6 5}$ & 0.65 & 0.26 & 0.68 & 0.01 & 0.015 & 0.07 & 0.05 & 0.01 & 0.08 & - \\
\hline $\mathbf{1 0 9 5}$ & 1.02 & 0.24 & 0.35 & 0.011 & 0.022 & 0.03 & 0.04 & 0.01 & 0.06 & - \\
\hline $\mathbf{1 5 4 1}$ & 0.41 & 0.21 & 1.46 & 0.01 & 0.011 & 0.08 & 0.06 & 0.02 & 0.1 & - \\
\hline $\mathbf{4 0 3 7}$ & 0.37 & 0.22 & 0.79 & 0.014 & 0.01 & 0.05 & 0.06 & 0.23 & 0.08 & - \\
\hline $\mathbf{4 1 4 0}$ & 0.41 & 0.25 & 0.85 & 0.013 & 0.009 & 0.99 & 0.05 & 0.22 & 0.09 & - \\
\hline
\end{tabular}

Table 3-1: Chemistry of fastener grade steel alloys used in testing. 


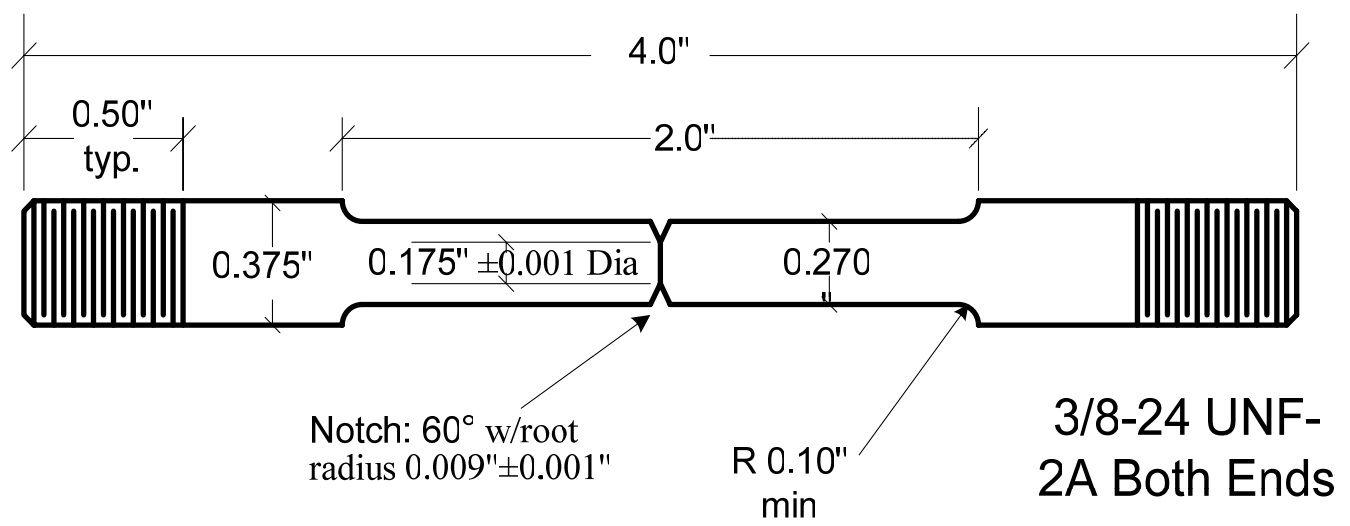

Figure 3-1: Drawing and dimensions of the notched tensile sample.

\subsection{2: 10B21 Heat Treatment}

A curve showing the relationship between tempering temperature and hardness after water quenching was generated for the 10B21 alloy. This is shown below in Figure 3-2. From Figure 3-2, it can be seen that a maximum hardness of HRc47 was achieved with a fully quenched sample. A hardness specified at HRc40 was chosen for testing of the 10B21 alloy and Jantom Precision, Inc. obtained the heat treatment. Although specific tempering temperatures were not received, extrapolation of the data from figure $3-2$ shows that a tempering temperature of $316^{\circ} \mathrm{C}\left(600^{\circ} \mathrm{F}\right)$ will produce the required hardness of HRc40. 


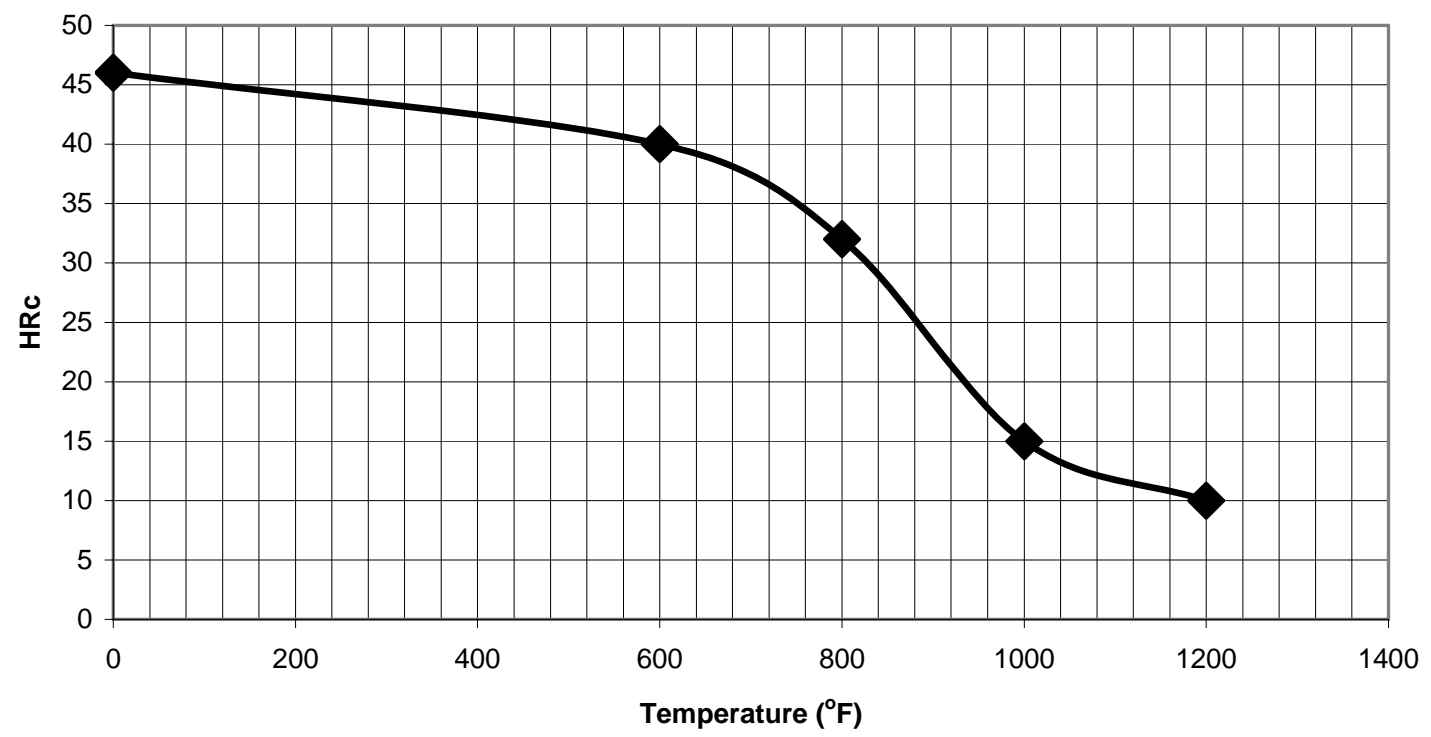

Figure 3-2: Tempering curve for 10B21 alloy used in experiments.

\subsection{3: 1541 Heat Treatment}

A tempering temperature versus hardness curve measured for 1541 is shown below in Figure 3-3. The curve shows a maximum attainable quenched hardness value of HRc53. Jantom Precision also obtained the quenching and tempering heat treatments for the test specimens of this alloy. Two hardness values of the tensile specimens were specified, HRc35 and 45, which required tempering temperatures of approximately $399^{\circ} \mathrm{C}\left(750^{\circ} \mathrm{F}\right)$ and $274^{\circ} \mathrm{C}\left(525^{\circ} \mathrm{F}\right)$, respectively. 
HRc Vs Tempering Temperature (1541, 3/8" dia., 2 hr. temper )

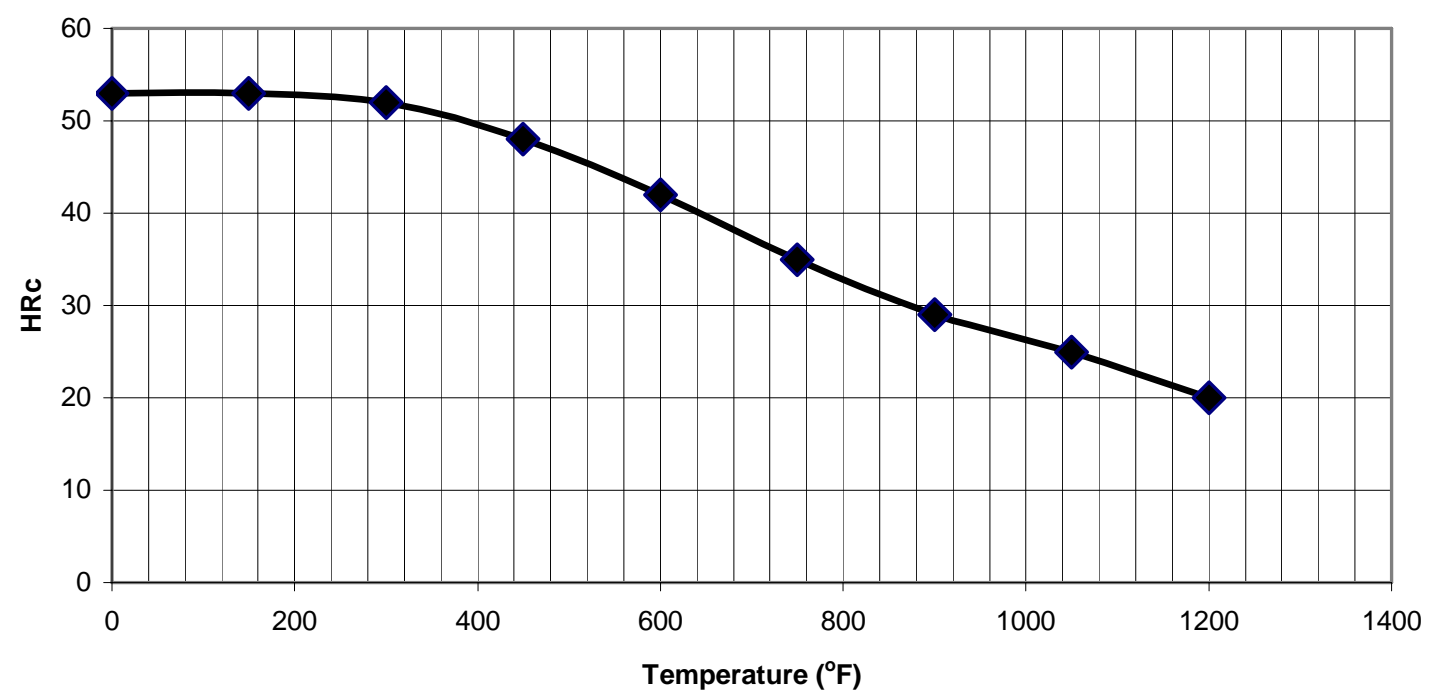

Figure 3-3: Tempering curve for alloy 1541 used in experiments.

\subsection{4: 1038 Heat Treatment}

Samples of the 1038 alloy were initially heat treated through Jantom. Order specifications were two specified hardness levels of HRc35 and 45 and a microstructure of quenched and tempered martensite. Upon examining the alloy, it was discovered that the microstructure was not homogenous martensite, but consisted of martensite with ferrite and pearlite around the prior austenite grain boundaries, as shown in Figure 3-4. This alloy was most likely oil-quenched, where the cooling rate was insufficient for a complete martensitic transformation and the samples were tempered at lower tempering temperatures than those utilized for fully martensitic structures. 


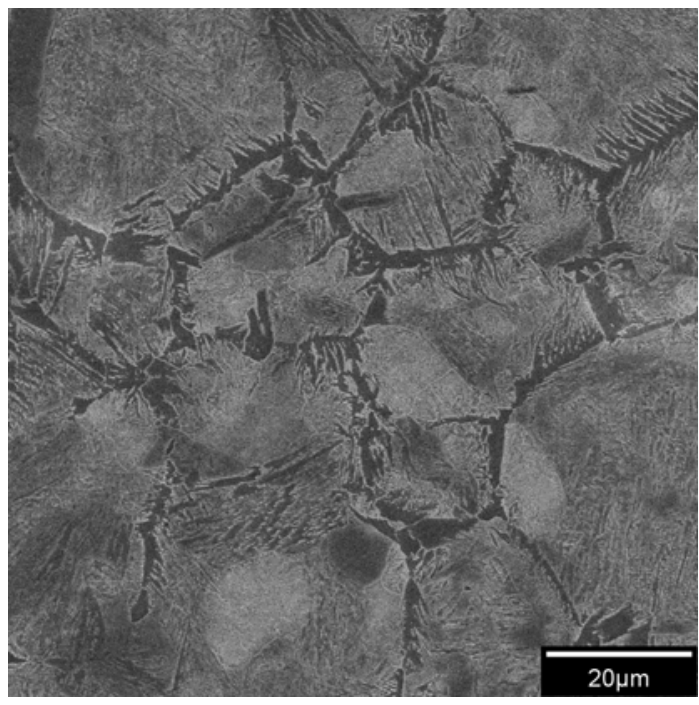

a.

Figure 3-4: Images showing the heterogeneous microstructures of the 1038 samples heat treated through Jantom Precision. a. SEM image, dark phase is ferrite, gray phase is prior austenite grains transformed to martensite. b. Optical image, scale bar is 20 um, light phase is again ferrite and darker phase martensite.

The heterogeneous microstructure that was produced resulted in very poor notch properties and could not be used in the RSL testing. An attempt was made to re-heat treat the samples, after the notch had been ground in, with the samples encapsulated in a glass tube under an argon atmosphere. This resulted in undesirable quench cracking and distortion of the sample following the quenching treatment, and as a result, these samples were discarded and new 1038 samples were generated.

Heat treatment of the new 1038 samples was performed at Michigan Tech. Several different quenching media were explored to produce a fully martensitic microstructure in the 1038 steel as well as the 1065 and 1095 steels. These steels all have very low hardenability due to their lack of alloying elements. The different quench media included various blends of a polymer quenchant at ambient and elevated temperatures, standard oil quenching and water quenching at ambient temperature and 
elevated temperatures. The oil quench was not severe enough to produce fully martensitic structures, but on the other hand the ambient water quench was too severe and quench cracking resulted longitudinally along the samples. The polymer-water quench was too unpredictable; therefore a quench into water at temperature of $43^{\circ} \mathrm{C}$ was utilized. This heat treatment produce a fully martensitic structure with only one sample lost to quench cracking.

The rough machined and threaded 1038 samples were placed in iron filings to prevent oxidation and de-carburization, then austenitized at $858^{\circ} \mathrm{C}$ for two hours, followed by the $43^{\circ} \mathrm{C}$ water quench. Samples were then placed in iron filings and tempered at two different tempering temperatures, $300^{\circ} \mathrm{C}$ or $400^{\circ} \mathrm{C}$, with target hardness values of 45 and $35 \mathrm{HRc}$, respectively.

\subsection{5: 4140, 1095 and 1065 Heat Treatment}

\subsubsection{A: Austempering}

Both austempering and quench and tempering heat treatments were performed on the 1065, 1095 and 4140 steels. The austempering treatment was initially attempted at Michigan Tech, but due to the difficulty in producing a homogenous bainitic structure, it was decided that a professional heat treatment facility should perform the austempering. Applied Process, Inc volunteered to perform these heat treatments. An austenitizing temperature of $871^{\circ} \mathrm{C}$ was selected for the 1095 and 1065 steel and a hold time of 36 minutes was utilized. Salt bath temperatures and hold times for the 1095 steel were $204^{\circ} \mathrm{C}$ for 142 minutes, $325^{\circ} \mathrm{C}$ for 86 minutes and $384^{\circ} \mathrm{C}$ for 53 minutes, while bath temperatures for the 1065 were $325^{\circ} \mathrm{C}$ for 86 minutes and $362^{\circ} \mathrm{C}$ for 41 minutes. The 
desired hardnesses of the 1095 samples were 40, 50 and 60 HRc, and for the 1065, 40 and $50 \mathrm{HRc}$. The austenitizing of the 4140 took place in a furnace at $880^{\circ} \mathrm{C}$ for 36 minutes, followed by salt bath quenching at $298^{\circ} \mathrm{C}$ for $52 \mathrm{~min}$., $327^{\circ} \mathrm{C}$ for $46 \mathrm{~min}$. and $357^{\circ} \mathrm{C}$ for 40 minutes, to achieve the desired hardness values of 30, 40 and 50 HRc. Figure 3-5 shows an image of the rack used during the austempering of the steel samples.

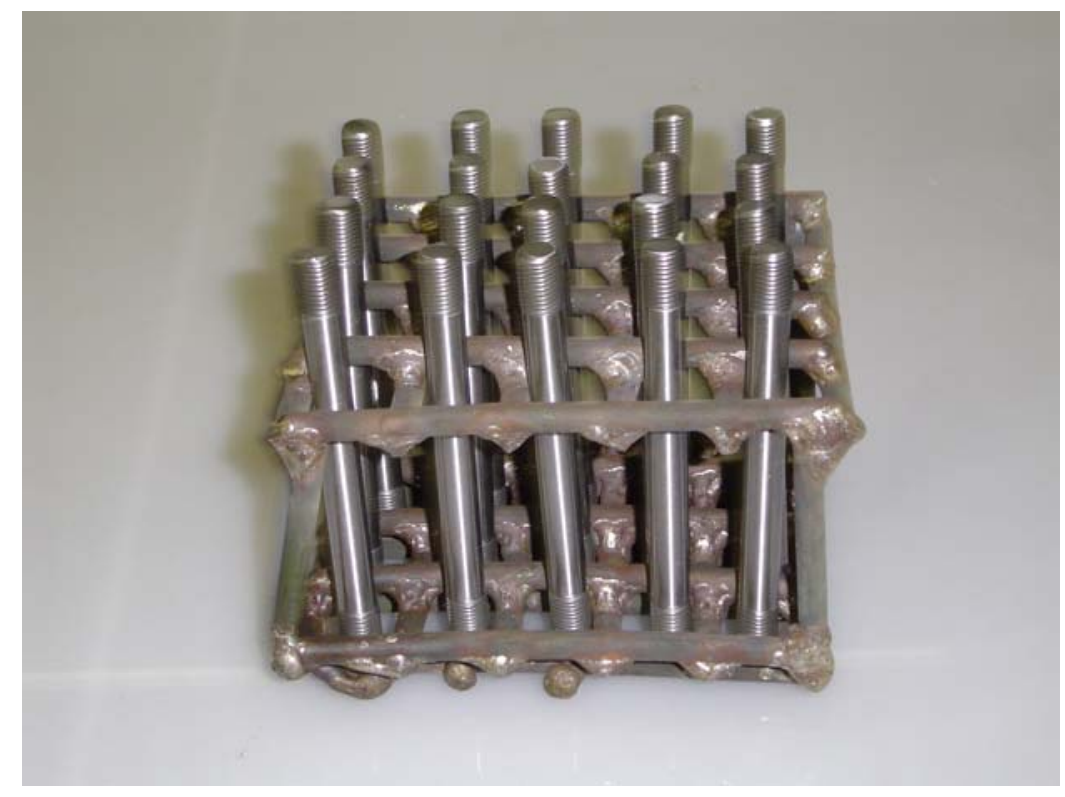

Figure 3-5: Rack and samples used in the austempering of the 4140, 1095 and 1065 steel tensile samples.

\subsubsection{B: Quenching and Tempering}

Samples of 1065, 1095 and 4140 were also quenched and tempered to produce martensitic structures. Quenching and tempering of the 4140 was performed through Jantom Precision, with specified hardness values of 35, 45 and 55 HRc. The quench and temper heat treatments for the high carbon, 1065 and 1095, steels were completed at Michigan Tech. The austenitizing of both the 1095 and 1065 was done at $815^{\circ} \mathrm{C}$ for 2 hours in iron filings, and then quenched into the $43^{\circ} \mathrm{C}$ water. All samples were quenched 
into liquid nitrogen for 10-15 minutes, to ensure that any retained austenite present would be transformed to martensite. An increase in hardness value of 1-2 HRc was observed after the nitrogen quench, signifying the transformation of small amounts of retained austenite to martensite. The tempering temperatures used for the 1065 were $260^{\circ} \mathrm{C}$ and $510^{\circ} \mathrm{C}$, and $260^{\circ} \mathrm{C}, 410^{\circ} \mathrm{C}$ and $550^{\circ} \mathrm{C}$ for the 1095 . The low tempering temperature of $260^{\circ} \mathrm{C}$ for the 1065 was too low and resulted in notch sensitivity. These 1065 samples were re-tempered at $400^{\circ} \mathrm{C}$ for 1 hour.

\subsection{6: 4037 Dual Phase Heat Treatment}

A dual phase heat treatment was completed on the 4037 to produce a microstructure of ferrite in a martensite matrix at two different hardness levels. The treatment started with austenitizing at $855^{\circ} \mathrm{C}$ for $2 \mathrm{hrs}$, followed by oil quenching, to produce a fully martensitic structure. The 4037 samples were then re-heated to $760^{\circ} \mathrm{C}$ (in the two phase alpha plus gamma region) and held for 2 hours, before the samples were again quenched into water. All samples were packed into iron chips to prevent oxidation at the elevated temperatures. Following the final quench the specimens had a hardness close to $47 \mathrm{HRc}$. Tempering temperatures of $150^{\circ} \mathrm{C}$ and $350^{\circ} \mathrm{C}$ and holding times of 2 hours were used to temper the martensite.

\section{2: Tension Testing}

\subsection{1: Rising Step Load (RSL) Procedure}

The ASTM F 1624 - 95 testing method, (“Standard Test Method for Measurement of Hydrogen Embitterment in Steel by the Incremental Loading Technique,”) was utilized in the testing for hydrogen assisted cracking susceptibility. The notched samples previously mentioned and shown in Figure 3-1 were used in the testing. 
A Fracture Diagnostics, Inc., Rising Step Load tensile machine, capable of a 10,000 lbs capacity was used for all of the SCC testing.

Initially a continuous load is applied to the sample, in ambient air, until the specimen fails. This test was performed on two to three samples and the failure load of the samples was averaged to obtain the critical load required to rupture the specimen, $P_{\mathrm{c}}$ [28]. Once $P_{\mathrm{c}}$ is established, a rising step load test is executed on a sample in air, using $P_{\mathrm{c}}$ as the maximum load and increasing in increments of $5 \%$ of $P_{\mathrm{c}}$ for one hour intervals until the specimen fails.

Specimens are then tested in a salt water solution to measure their resistance to SCC. The tensile samples are first coated with epoxy on all of the gauge length, with the exception of the notch. This assists in localizing hydrogen adsorption at the notch. Specimens are then mounted within Styrofoam cups, filled with a 3.5\% NaCl-water solution, and loaded into the RSL. A cathodic potential is supplied between a platinum electrode and the sample and is monitored and fixed at -1.2 Volts vs. a Standard Calomel Electrode (S.C.E). Three samples are tested using $P_{\mathrm{c}}$ as the initial fracture load for the $P_{\mathrm{i} 1}$ loading profile, which is shown in Table 3-2, and the fracture loads obtained from those three samples are averaged and used as the fracture load for the $P_{\mathrm{i} 2}$ testing. This process is repeated for the $P_{\mathrm{i} 2}$ and $P_{\mathrm{i} 3}$ testing protocols. 


\begin{tabular}{cccc}
$\begin{array}{c}\text { Load } \\
\text { increment }\end{array}$ & \multicolumn{3}{c}{ Step Time (hours) } \\
$\mathbf{( \% )}$ & $\# \mathbf{1}$ & $\# \mathbf{2}$ & $\# \mathbf{3}$ \\
5 & 1 & 1 & 1 \\
10 & 1 & 1 & 1 \\
15 & 1 & 1 & 1 \\
20 & 1 & 1 & 1 \\
25 & 1 & 1 & 1 \\
30 & 1 & 1 & 1 \\
35 & 1 & 1 & 1 \\
40 & 1 & 1 & 1 \\
45 & 1 & 1 & 1 \\
50 & 1 & 1 & 1 \\
55 & 1 & 2 & 4 \\
60 & 1 & 2 & 4 \\
65 & 1 & 2 & 4 \\
70 & 1 & 2 & 4 \\
75 & 1 & 2 & 4 \\
80 & 1 & 2 & 4 \\
85 & 1 & 2 & 4 \\
90 & 1 & 2 & 4 \\
95 & 1 & 2 & 4 \\
100 & 1 & 2 & 4 \\
Total Hours & $\mathbf{2 0}$ & $\mathbf{3 0}$ & $\mathbf{5 0}$
\end{tabular}

Table 3-2: Loading profiles used in RSL testing of notched tensile specimens.

Differences in $P_{\mathrm{i} 2}$ and $P_{\mathrm{i} 3}$ failure loads were fairly minimal in the initial testing, therefore the $P_{\mathrm{i} 3}$ protocol was not employed for some of the subsequent tests.

\section{3: Characterization}

\subsection{1: Hardness, Metallography and Fractography}

Following RSL testing, four specimens from each lot were cut transverse through the sample, mounted in bakelite, and then polished with silicon carbide to 600 grit. Vickers hardness measurements were taken through the cross section of each sample.

Vickers hardness values from the four samples were averaged and used as the hardness of each lot tested. One of the mounted samples from each lot was polished to a 0.05 micron 
alumina finish and etched with 2\% Nital for optical and SEM examination of the microstructure. A Hitachi S-4700 FE-SEM was used to obtain a fine detailed image of the microstructure of some selected samples that could not be easily resolved with the optical microscope.

The fracture surface, resulting at the notch of each tensile specimen, of selected specimens was cut from the sample and examined using the JOEL-6400 scanning electron Microscope. The entire fracture surface was analyzed and images were typically captured at a magnification of 20x, to show the macroscopic features, and 1500x to show microscopic features.

\subsection{2: X-ray Diffraction}

X-ray diffraction data was collected for the 4140, 1065 and 1095 steels using a Sintag XDS-2000 machine. One specimen per lot of each of the three alloys previously listed was cut and mounted in bakelite, polished to 0.05 micron then etched with $2 \%$ Nital. Three times the samples were polished using the 0.05 micron alumina and etched, a common procedure for assuring minimal strain effects on the metal surface. All scans were collected using the scan parameters as follows; tube divergence slit of $1 \mathrm{~mm}$ and scatter slit of $2 \mathrm{~mm}$, detector receiving slit of $0.3 \mathrm{~mm}$ and scatter slit of $0.5 \mathrm{~mm}$, step size of $0.3^{\circ}$, and a scan time per step of 20 seconds. The 4140 scans were collected over the scanning ranges of $61^{\circ}$ to $68^{\circ}$ and $78^{\circ}$ to $87^{\circ}$. This range produces two peaks, the ferrite 200 and 211 peaks, which were used to calculate the particle size. The scan range for the 1065 and 1095 was $35^{\circ}$ to $105^{\circ}$, a large scan that included carbide, ferrite and possible austenite peaks. 
Each peak observed in the scans was identified and curve fit with a Pearson 7 algorithm using the Sintag program, dmsnt. If there was a $\mathrm{K}_{\alpha 2}$ peak visible (typically highly tempered specimens), then both peaks were fit with the Pearson 7 algorithm. Once each peak was fit with a curve, the area under the peak, FWHM, peak position, and intensity were automatically calculated by the program. Once these properties were known, the ferrite crystallite size for all samples was calculated and volume fraction of austenite and carbon content in the austenite were calculated if austenite was present. 


\section{Chapter 4: Results and Discussion}

\section{1: Rising Step Load (RSL) Threshold Stress Results}

The alloys and hardness of all alloys tested under the RSL protocols specified in the previous section are shown below in Table 4-1, where QT signifies a quench and temper treatment, AT represents austempering treatments and DP notes the dual phase heat treated samples. All fracture loads and calculated air and hydrogen threshold fracture stresses are listed in the appendices. The quenched and tempered 1038, 1541, 4140 specimens at a given hardness are compared to the 4037 quenched and tempered samples, obtained in previous work [4], to see variations in threshold stress levels with differences in compositions. Quenched and tempered samples of the alloys 1038, 1065 and 1095 are utilized to see how an increase in carbon content of the steel affects the behavior of the steel when hydrogen charged. The results from the 10B21 specimen can be used to see changes in threshold stress with both alloying content and carbon content, since the alloy is a plain carbon steel with a small amount of boron. Quenched and tempered data are compared to austempered data collected with the 4140, 1065 and 1095

alloys. Finally, the 4037 dual phase treatment using a step anneal processing is compared to 4037 dual phase steel treated with a continuous cooling process obtained in earlier work [4]. 


\begin{tabular}{|c|c|c|c|}
\hline \multirow{2}{*}{ Alloy } & \multicolumn{3}{|c|}{ Hardness (HVN) } \\
\cline { 2 - 4 } & Low & Medium & High \\
\hline 10B21 (QT) & - & 400 & - \\
\hline 1038 (QT) & 347 & - & 492 \\
\hline 1065 (QT) & 361 & - & 491 \\
\hline 1065 (AT) & 361 & - & 529 \\
\hline 1095 (QT) & 334 & 481 & 584 \\
\hline 1095 (AT) & 435 & 483 & 776 \\
\hline 1541 (QT) & 366 & - & 460 \\
\hline 4037 (DP) & 389 & - & 473 \\
\hline 4140 (QT) & 419 & 466 & 519 \\
\hline 4140 (AT) & 448 & 509 & 532 \\
\hline
\end{tabular}

Table 4-1: Hardness value of all alloys tested. $\mathrm{QT}=$ quench and temper, $\mathrm{AT}=$ austemper, $\mathrm{DP}=$ dual phase

Two lots of specimens, the 1095 QT and AT with hardness values of 584 and 776 respectively, could not be utilized in the RSL testing due to their notch sensitive behavior in air. The notch sensitivity for the various steels can be estimated from Equation 2-4. Table 4-2 displays the sensitivity for the austempered 1095 specimens. A notch severity of one signifies the maximum effect a notch can have on a member. The value of the notch sensitivity factor for the high hardness 1095 austempered specimen is high enough to justify it as notch sensitive due to its severe lack of ductility. Results from the sensitivity of the two lots signifies that a maximum hardness of approximately $550 \mathrm{HVN}$ should not be exceeded in notched steel fasteners to ensure that the fastener can withstand adequate loading in air.

\begin{tabular}{|c|c|c|c|}
\hline Hardness & UTS (Mpa) & $\alpha(\mathbf{m m})$ & $\mathbf{q}$ \\
\hline $\mathbf{4 3 5}$ & 1443 & 0.04785 & 0.83 \\
\hline $\mathbf{4 8 3}$ & 1590 & 0.04019 & 0.85 \\
\hline $\mathbf{7 7 6}$ & 2487 & 0.01797 & 0.93 \\
\hline
\end{tabular}

Table 4-2: Notch sensitivity calculations for austempered 1095 specimens. 
The data for 4037 was extrapolated from previous work [4]. There was a slight difference in the gauge diameter for the 4037 and the 4140 austempered specimens utilized in RSL testing. The diameter for these samples was 0.25 ” while all other diameters were 0.27 ”. This slight discrepancy resulted in a lower stress concentration at the notch root for the smaller diameter samples. Equations 2-1 and 2-2 were used to calculate the difference in stress concentrations for the different gauge diameters. The values of the calculated stress concentration factor were 3.046 and 3.100 for the 0.25 ” and 0.27 ” gauge diameters, respectively. This calculated difference is about $2 \%$. The stress concentration factor listed in the ASTM standard F519 is 3.1 [34], which corresponds to a v-notch with a gauge diameter of $0.25 ”$ An increase in stress concentration should increase the fracture load in air but decrease the threshold stress in the salt solution. To verify this, remaining specimens of 4140 quenched and tempered to 519 HVN, were machined from the 0.27 ” to 0.25 ” gauge. Two samples were tested in air and three in salt water under the $\mathrm{P}_{i 1}$ protocol. The average fracture load of the air specimens was $371 \mathrm{lbs}$ higher for the 0.27 ” diameter and 316 lbs lower for the salt specimens. Samples were tested with a lower hardness value that verified these results. All quenched and tempered 4037 and austempered 4140 data were corrected to account for the difference in the gauge diameter, utilizing the data collected for the 4140.

An overall relationship between the fracture strength in air and the nominal threshold stress obtained during hydrogen charging as a function of hardness can be seen in Figure 4-1. The general trend for the strength in air shows a linear trend of increasing fracture strength with increasing hardness, as would be expected for steels with increasing yield strengths. There appears to be significant scatter in the air for some 
samples. This is due to microstructural and alloy variations and will be discussed further in the following sections.

Data for the environmental testing shows a decrease in nominal stress required to fracture the specimen as the hardness of the steel is increased. This behavior was explained by Troiano [2]. If there exists a critical hydrogen concentration for hydrogen induced cracking of a given metal and microstructure, then there is a correlating critical or threshold stress required to drive the hydrogen to the notch root and initiate void nucleation. The required hydrogen concentration can be related to a critical interaction energy term corresponding to the sum of the three principal stresses at the notch root, which is also constant for a material. Plasticity, which decreases with increasing hardness of the material, will decrease the stress concentration at the notch root. Therefore as the yield strength of a metal increases, e.g. at higher hardness values, the stress concentration will increase at the notch root due to less plasticity, and the nominal stress required to reach the critical stress will decrease. This theory is only valid if the critical hydrogen concentration is supplied above the concentration in irreversible traps. Since a threshold stress was found during the charged RSL testing protocol, it appears that the critical concentration was met. The scatter in data for the hydrogen charged samples is related to differences in microstructure and alloy content for the steels tested. 


\section{Notched Threshold Stress Vs Hardness}

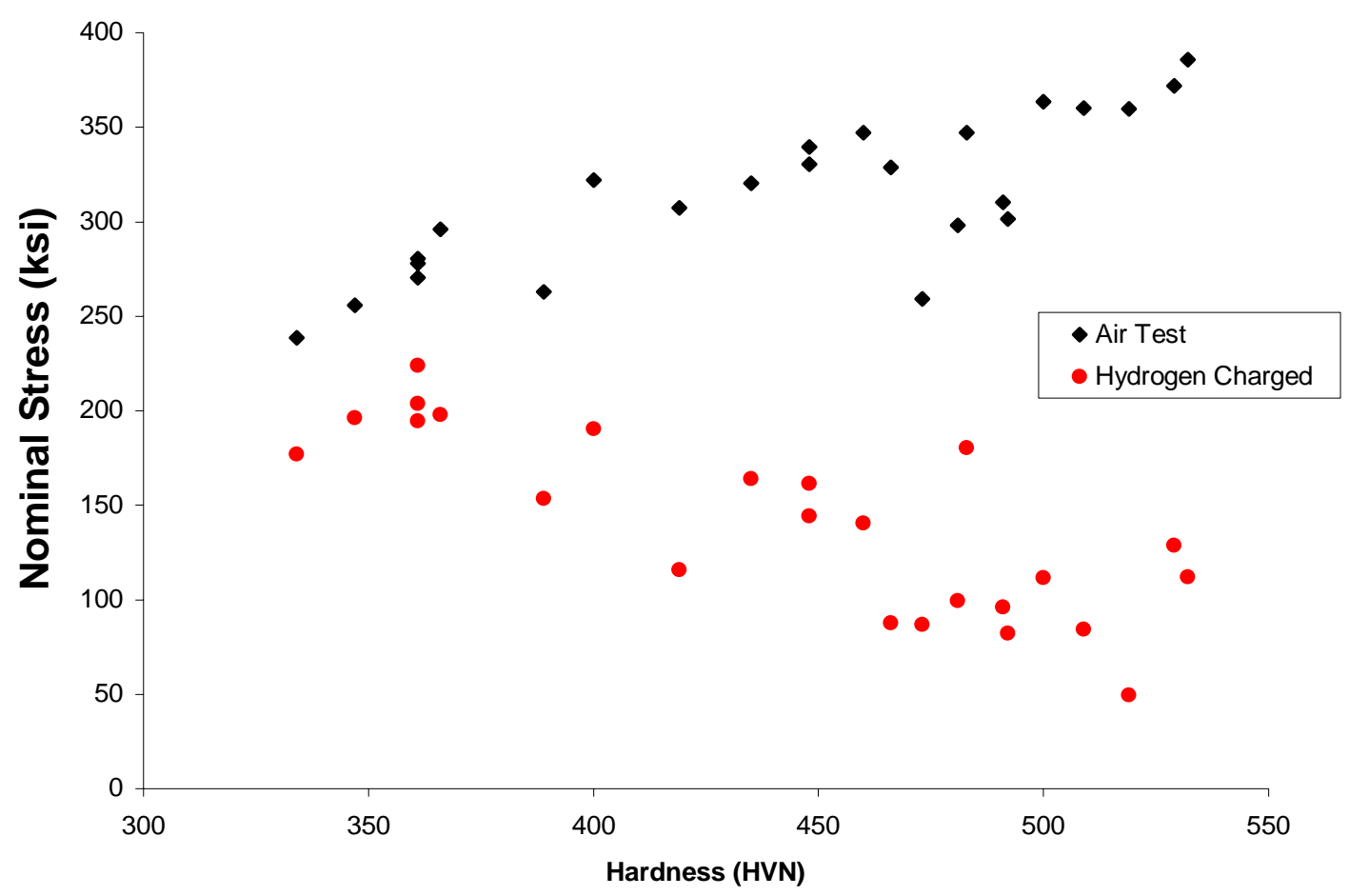

Figure 4-1: Hydrogen charged and uncharged data for all alloys, heat treatments and strength levels.

\subsection{1: Dual Phase}

A step annealing procedure, previously explained, was performed to modify the morphology of the ferrite phase. This procedure also induced higher residual stresses in the ferrite when martensite transformation occurred. Micrographs of the 4037 dual phase material heat treated by the continuously and step cooled processes can be seen in Figure 4-2. The difference in size of the ferrite particles is around a factor of 10 and can easily be seen in the photomicrographs. The total ferrite content was measured as 12 percent for the continuously cooled steel and 20 percent for the step annealed. This difference in ferrite content is attributed to an increase in ferrite nucleation sites when the specimen is step annealed. The steels could be heated to temper the martensite to similar hardnesses. 
The hardness of the martensite phase in the two step annealed dual phase steels was estimated using the rule of mixtures and a ferrite hardness of $150 \mathrm{HVN}$. The values were determined to be about 450 and $550 \mathrm{HVN}$ for the macrohardness values of 389 and 473, respectively.
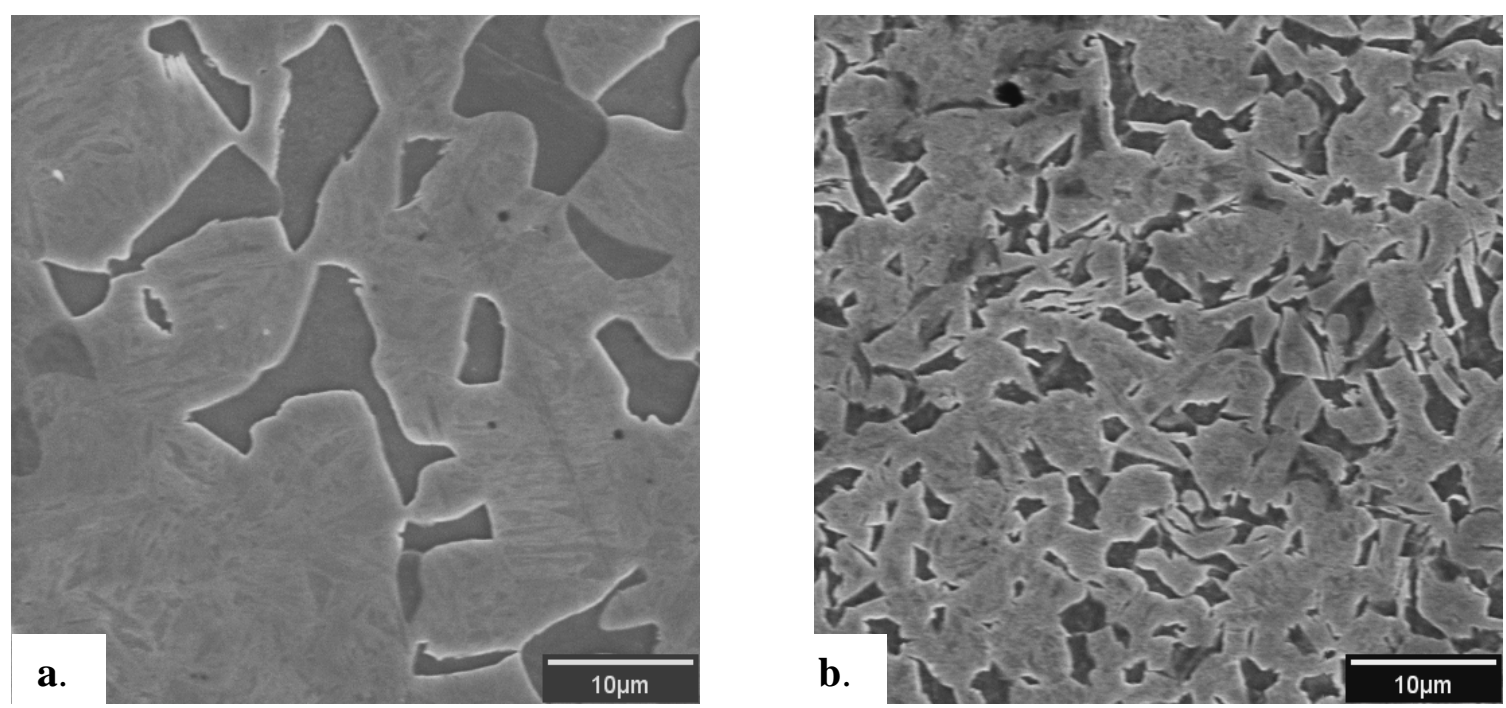

Figure 4-2: SEM images of a. continuously cooled 4037 dual phase steel, b. step annealed 4037 dual phase steel. Dark phase is ferrite and light phase martensite.

Comparisons of the threshold stress values of the 4037 quenched and tempered, continuously annealed and step annealed specimens are shown in Figure 4-3. It is apparent that there is virtually no change in hydrogen induced cracking susceptibility between the two dual phase steels. Both dual phase steels are also significantly more susceptible than a 4037 quenched and tempered steel structure.

Typically an increase in hardness resulted in an increased notched air fracture strength as can be seen in Figure 4-1. This trend was not observed for either of the dual phase treatments, which produced air fracture values of near 260 Ksi for all hardnesses. 
This signifies a notch sensitivity effect for these two dual phase steels, which is typically correlated to a lack of ductility or plasticity. The limited ductility of this material is most likely due to micro-void fracture along ferrite and martensite phase interfaces. This was observed optically by Grochowski [4]. The lack of ductility in the material also limited the critical threshold stress of the dual phase steels by intensifying the stress concentration at the notch root. Images of the fracture surface verify that a mostly brittle fracture mode occurred when testing the step annealed samples in air and in salt water at both hardness values. The fracture surfaces can be viewed in Figure 4-4. Both the low and high hardness samples showed a primarily cleavage fracture behavior in air with some dimple fracture. Micro-cracking can be observed in the higher hardness air sample, but not the lower. The fracture mode of the hydrogen charged low hardness material was a cleavage mode similar to that tested in air. The higher hardness hydrogen charged specimen produced a failure mode of both brittle cleavage and intergranular, and again micro-cracking can be seen.

The RSL data and brittle fracture behavior implies that this particular grade and heat treatment of steel is not suitable to fastener applications where thread roots and radius create stress concentrations similar to the ones observed during these experiments. It would be interesting to study the notch and SCC behavior of classical dual phase steel consisting of a ferrite matrix with small martensite islands. 
Threshold Stress Vs Hardness

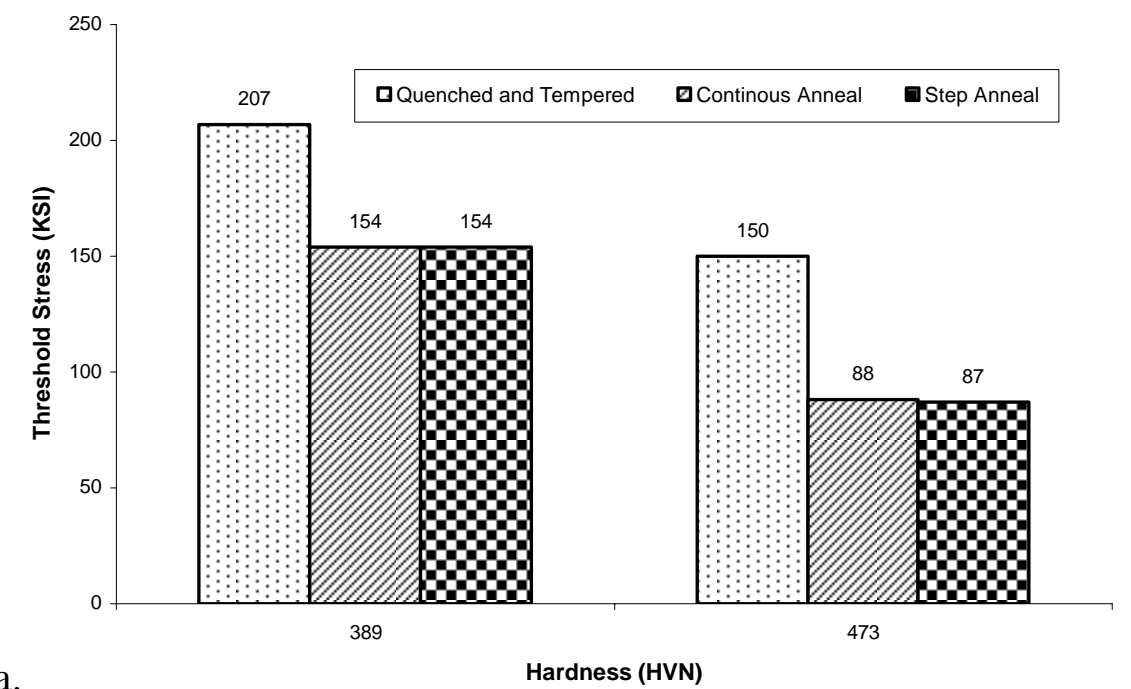

a.

Hardness (HVN)

Percent Fracture Strength Vs Hardness

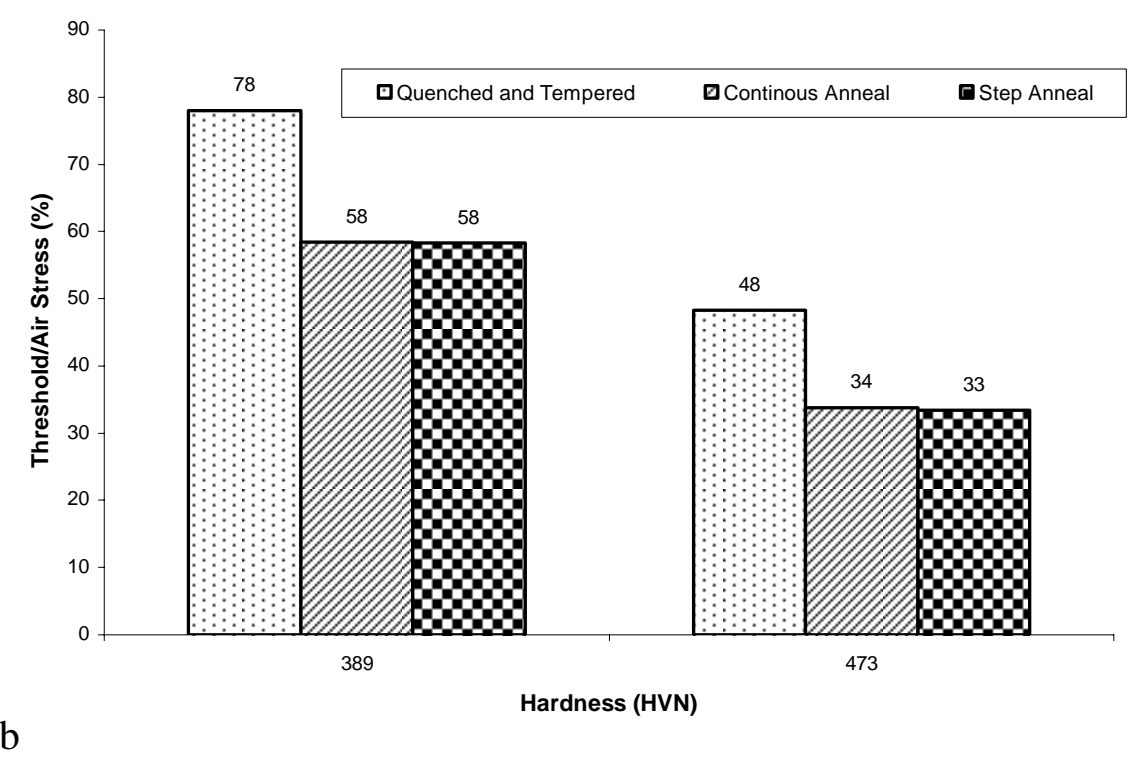

Figure 4-3: Bar charts displaying behavior of dual phase structures compared to quenched and tempered for: a. Threshold stress, b. Threshold fracture stress as a percent of air fracture stress. (Quenched and tempered and IA 4037 data from [4]) 

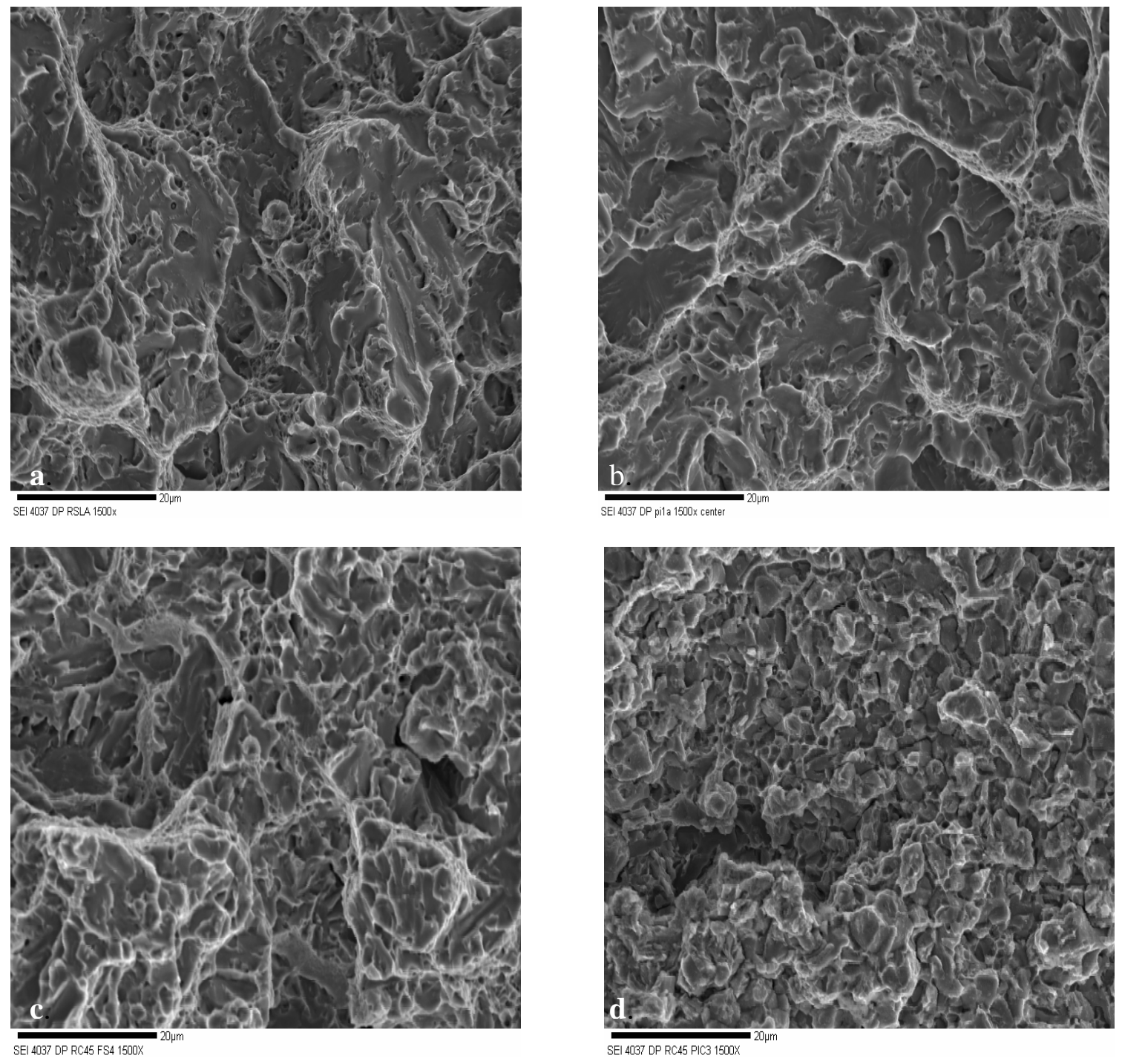

Figure 4-4: Fractographs of 4037 step annealed dual phase steel: a. Low hardness (389 HVN) air sample,

b. Low hardness hydrogen charged sample, c. High hardness (473 HVN) air sample, d. High hardness hydrogen charged sample. Scale bars are 20 microns

\subsection{2: Effects of Alloying elements and Carbide Content on HIC}

When first examining all the data it should be noted that there is a slight change in notched air fracture stress trends between the quenched and tempered plain carbon steel and all the other data including austempered plain carbon steels. This difference can be 
seen in Figure 4-5; both are linear, but the slope for the quenched and tempered carbon steels in slightly less and the intercept a bit higher. The error bars in Figure 4-5 represent the high and low values of individual fracture tests. There is not enough scatter to attribute the differences to an error effect, so the difference must be explained by microstructural features. The fractography features of the high hardness quenched and tempered carbon steels are not much different from that of the other steels, with the exception of a possible increase in micro-cracking. This micro-cracking may result in a higher notch sensitivity of the carbon steel, which decreased the slope of the air fracture trend. Some carbide particles were not dissolved during the austenitizing of the carbon steels. These carbides can be seen optically in the 1095 quenched and tempered steel and appear to be approximately $1 \mathrm{um}$ in diameter. Nucleation of micro-cracks most likely occurs at the un-dissolved carbide-matrix interface. Fractographs of the high and low hardness 1038 air fracture specimens and an optical image showing un-dissolved carbides are displayed in Figure 4-6. 
Air Fracture Strength Vs. Hardness

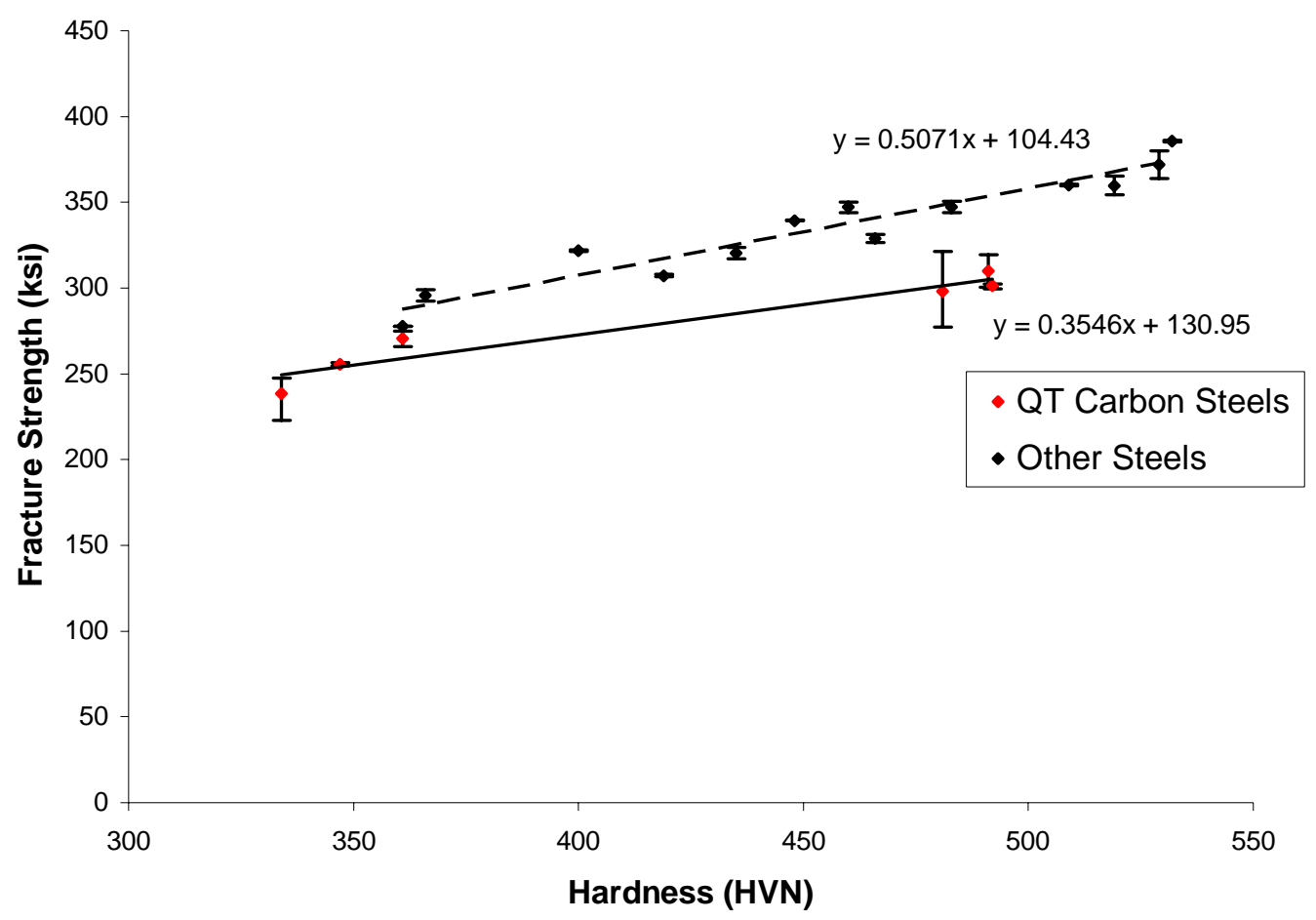

Figure 4-5: Plot showing the difference in uncharged fracture strength of the quenched and tempered carbon steels versus the alloyed steels. 


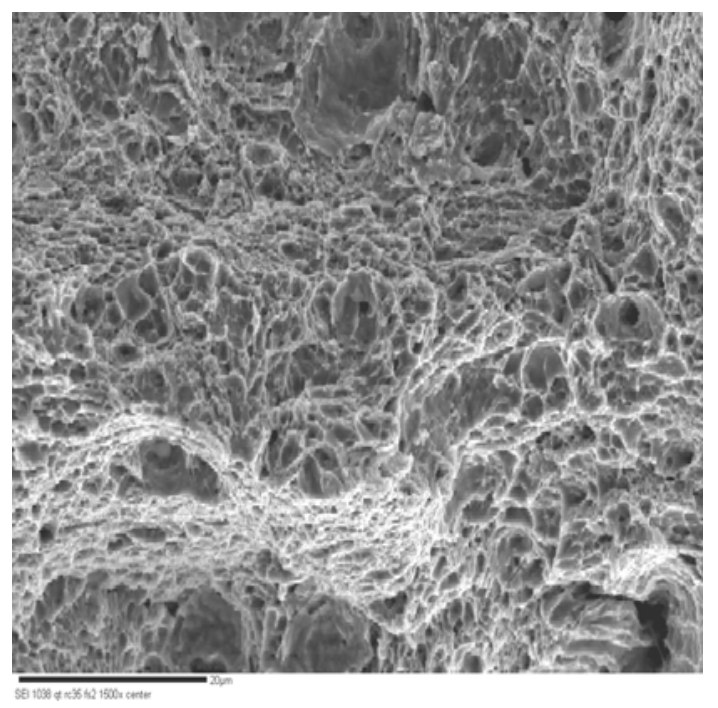

a.

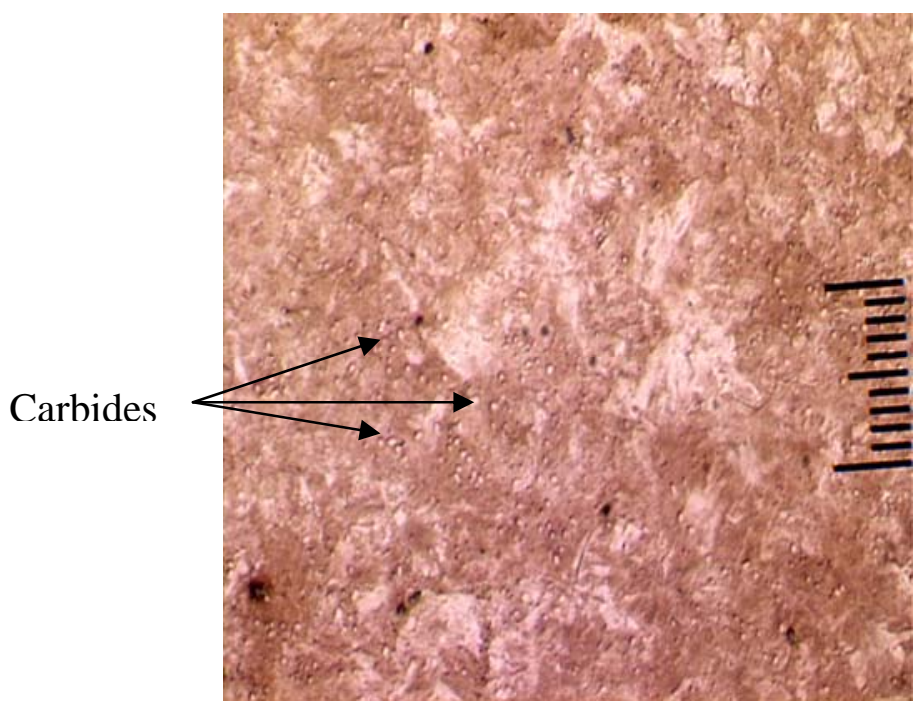

c.

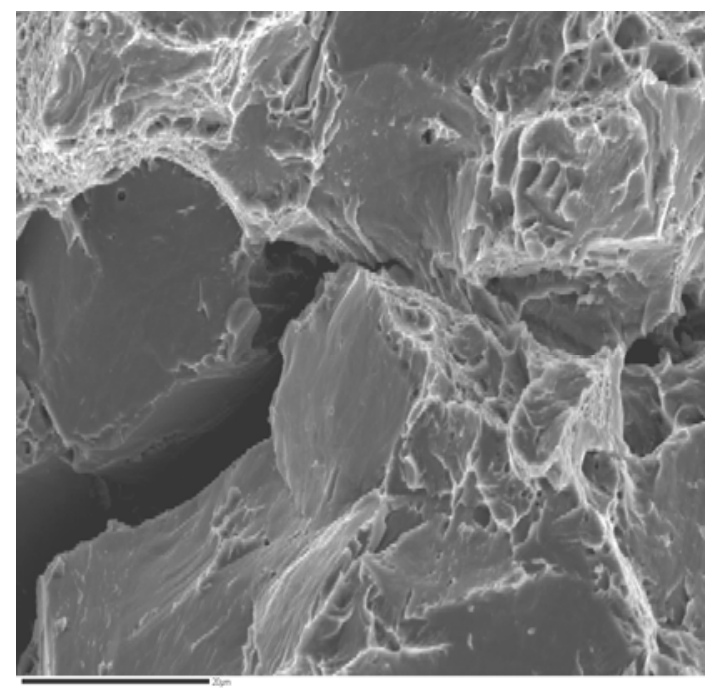

b.

Figure 4-6: SEM fracture surface of the 1038 uncharged specimens and optical image of 1095 specimen a. 347 HVN, b. 492 HVN, c. 1095 optical image showing un-dissolved carbides. All scale bars are $20 u \mathrm{~m}$ 
The effect that carbon content has on threshold stress levels can be seen in Figure 4-7, which shows the threshold stress as a percent of the air fracture stress. The trend of a decreasing threshold as hardness increases was explained previously in terms of the plasticity of the steel at higher hardness values. There appears to be no effect of carbon, i.e. carbide content, on the steels at a given hardness level. This is due to the continuous saturation of the sample with hydrogen during RSL testing. Since carbides act as irreversible traps, once they are filled with hydrogen, they have no effect on limiting the amount of hydrogen that can diffuse to the maximum triaxial stress and only reversible traps can act to limit the threshold stress. If there is a limited hydrogen supply, it is likely that the steels with elevated carbon contents will have an amplified critical hydrogen concentration required for hydrogen induced cracking. 


\section{Percent Fracture Strength Vs Hardness (Carbon Steels)}

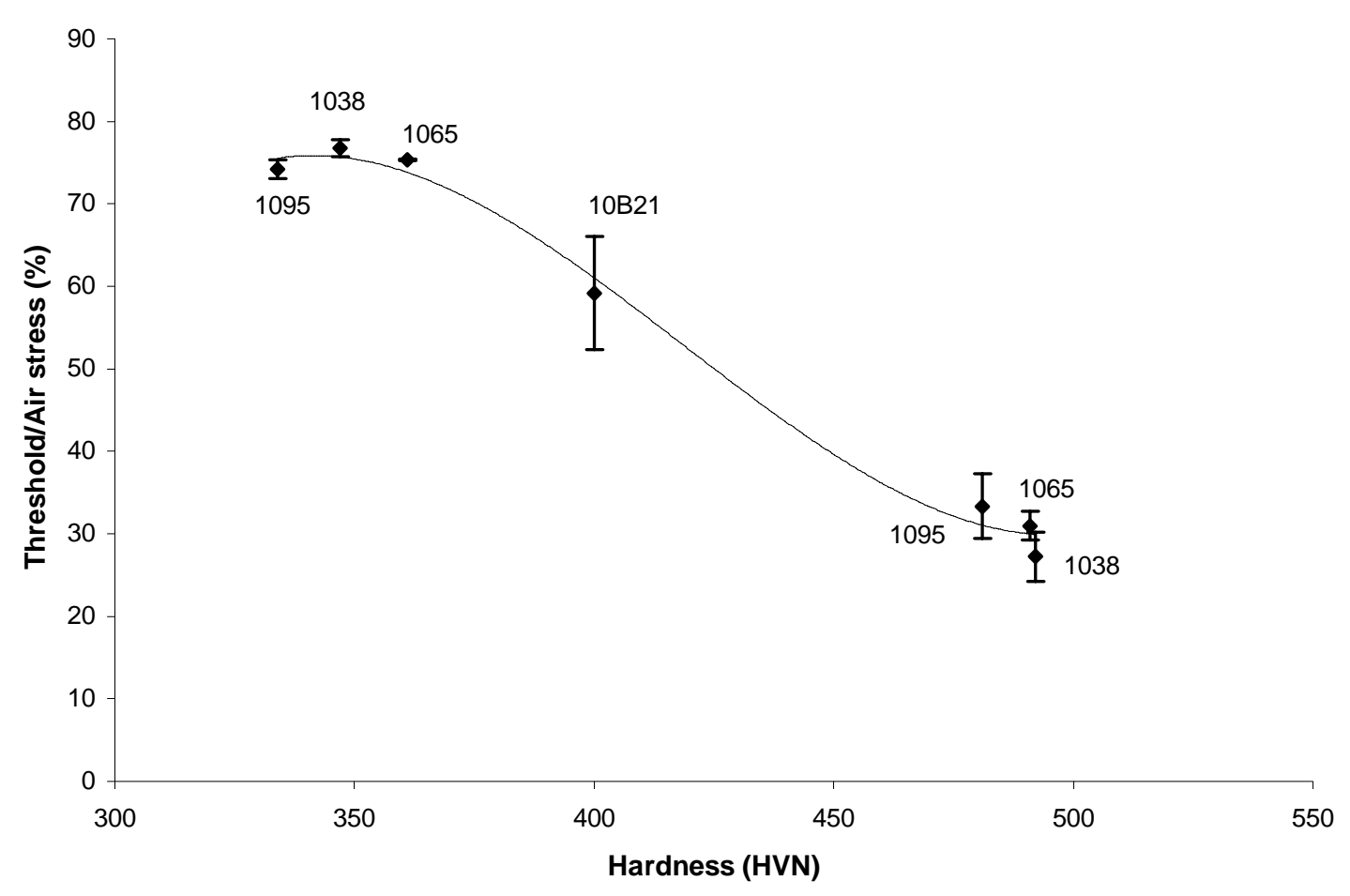

Figure 4-7: Data indicating no change in threshold stress as carbon content is increased. Error bars are the standard deviation of the threshold stress as a percent of the fracture strength.

It is difficult to assess the actual effects that alloying elements may have on resistance of a steel to hydrogen assisted cracking, since the commercial steel grades typically contain several alloying species. A systematic study varying only one alloying element at a time would be valuable to properly assess the resistance or susceptibility behavior. Still, we can make comparisons of the alloys tested. The elements that will be compared per grade are: boron in 10B21, manganese in 1541, molybdenum in 4037, and chromium in 4140. The RSL data from these alloys can be compared to the non-alloyed 1038 carbon steel. It should be noted that with the exception of the 10B21 steel, all 
grades have similar carbon contents. This was done purposely to separately study the effects of hardening alloy elements and carbon. The actual chemistry of each steel grade can be viewed in Table 3-1.

Rising step load data for the steels mentioned are shown in Figure 4-8 as a plot of the ratio of threshold stress to air fracture stress at the various hardness values. It is apparent that generally all the grades maintain a similar trend, with the exception of the 4140. This fact would lead one to the deduction that chromium is deleterious in hydrogen assisted cracking susceptibility. It is thought, however, that this increased susceptibility of the 4140 may be a result of temper embrittlement. Temper embrittlement is a result of impurity atom segregation at the grain boundaries that decreases the surface interfacial energy of the boundaries. Alloying elements like chromium, manganese and nickel are known to be a requirement to activate embrittlement by elements such as phosphorous, tin or arsenic [35]. Temper embrittlement occurs when steels are tempered at specified temperatures then slowly cooled. It is possible that some impurity segregation occurred in the 4140 during tempering and the fracture mode was intensified by the atomic hydrogen presented during RSL testing. This would explain the MVC fracture mode for the quenched and tempered 4140 in the air tests, but an intergranular path during hydrogen charging. Only the 4140 and 1038 hydrogen charged specimens produced an intergranular fracture path along prior austenite grains. Fractographs of the 4140 quenched and tempered steel are displayed as Figure 4-9. The fracture of the hydrogen charged samples were initially intergranular at the outer surface and micro-void fracture at the inner surface. 
Molybdenum is typically believed to make steel more resistant to temper embrittlement [23, 36] and may be why the 4037 steel showed a slight increase in resistance to HIC, as seen in Figure 4-8. The segregation of phosphorus, which typically causes temper embrittlement, can be retarded by additions of molybdenum to the steel [23]. Also, coherent molybdenum carbide particles that precipitate during tempering have been found to act as reversible traps for hydrogen and may reduce the susceptibility [26]. The reversible traps will not be saturated during the hydrogen charging and may play a role in the determination of the threshold stress. Boron and manganese appear to do little in modifying the hydrogen charged behavior for the heat treatments and grades of steels presented here. 


\section{Percent Fracture Strength Vs Hardness (Alloy Steels)}

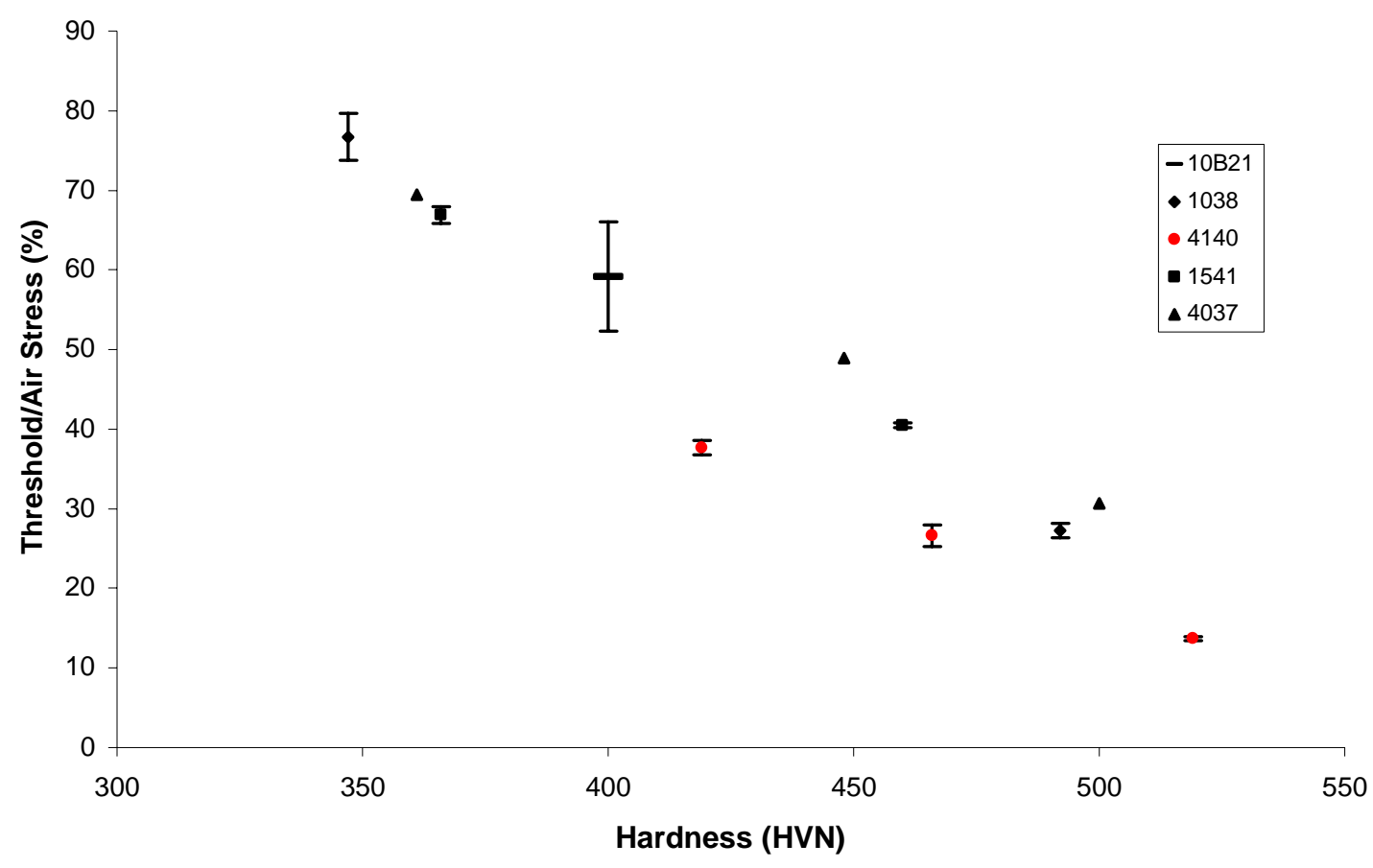

Figure 4-8: Plot showing the effects of the alloying content on HIC susceptibility. Red data points represent the 4140 that indicate a noticeable increase in susceptibility. (4037 data points from Grochowski [4]) 

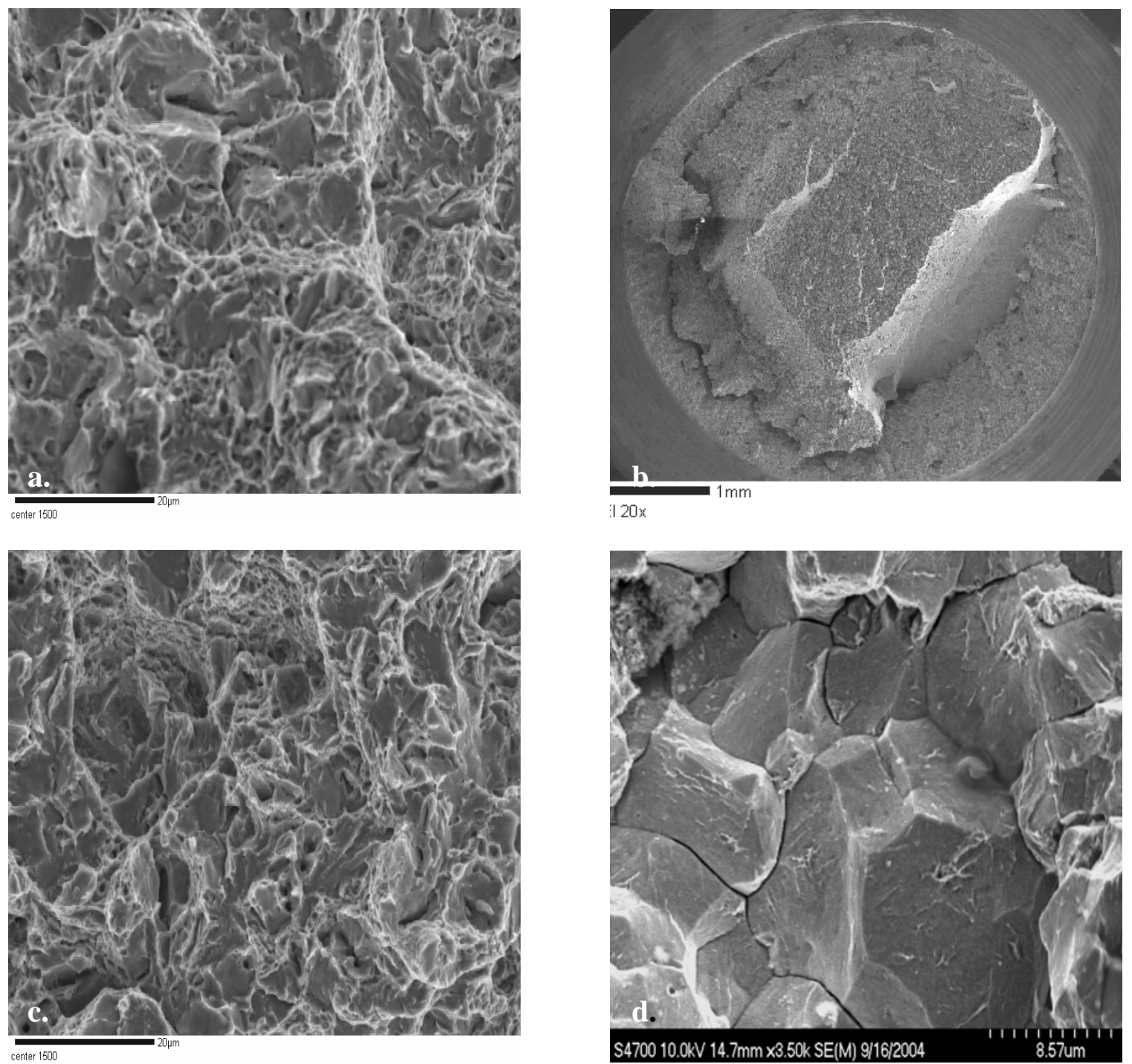

Figure 4-9: Fracture surfaces of quenched and tempered 4140 steel of hardness 466 HVN; a. Quasi-

cleavage air test sample (20 um scale bar), b. Low magnification charged sample showing outer and inner fracture areas (20x mag.), c. Quasi-cleavage fracture at center of charged specimen, d. Intergranular fracture at edge of charged specimen 


\subsection{3: Austempered Vs. Quenched and Tempered}

Three alloys were used for the comparison of a tempered martensitic structure to that of a bainitic structure with respect to their resistances to hydrogen induced cracking. The alloys were 4140, 1065 and 1095. Optical micrographs of martensitic and bainitic microstructures are shown in Figure 4-10. The hydrogen assisted cracking susceptibility of these grades is depicted in Figures 4-11 and 4-12. Both figures demonstrate an increased resistance to hydrogen attack for the bainitic steel structures of approximately $10-15 \%$. Although this difference is not great, it may be high enough to allow an increase in hardness of fasteners of 3-4 Rockwell C points. The difference is most prominent on the low hardness 1065 batch of samples, which showed only a 19\% drop in strength when charged with hydrogen. Also, the 1065 higher hardness austempered sample shows a slight increase in resistance to HIC, even though the hardness is about 40 HVN points higher than the martensitic specimen. The 4140 bainitic specimens displayed a continuous increase over the martensitic structure at all hardness levels. The fracture mechanisms, identified by the fractographs, were similar for quenched and tempered and austempered steels. The softer materials showed a quasi-cleavage fracture in air and a more ductile MVC in salt water, while the harder steels resulted in cleavage fracture in salt water and air. Representative SEM fracture images for all alloys can be viewed in the appendices.

Several reasons for the decreased susceptibility of the austempered structure could exist, but it is believed that the superior toughness of bainitic structures may contribute most to the decrease. The improved toughness of bainite may be attributed to the existence of small amounts of retained austenite that may also inhibit hydrogen diffusion 
to the stress concentration or the lack of strain related defects generated during quenching. It would be expected that grades of steel containing higher carbon concentrations would generate more retained austenite during isothermal transformations, resulting in increased resistance to SCC. Approximately $12 \%$ retained austenite was discovered in the high hardness (776 HVN) 1095 austempered steel. It is likely that all 1065 and 1095 austempered specimens contained a small amount of retained austenite that may have been below detectable levels within the x-ray diffraction procedure employed. Also, the structure is more homogenous and stress free following an isothermal treatment, as compared to a quenching heat treatment. Another reason for the increase in resistance may be the absence of temper embrittlement in austempered materials and a more uniform distribution of alloy and impurity elements.
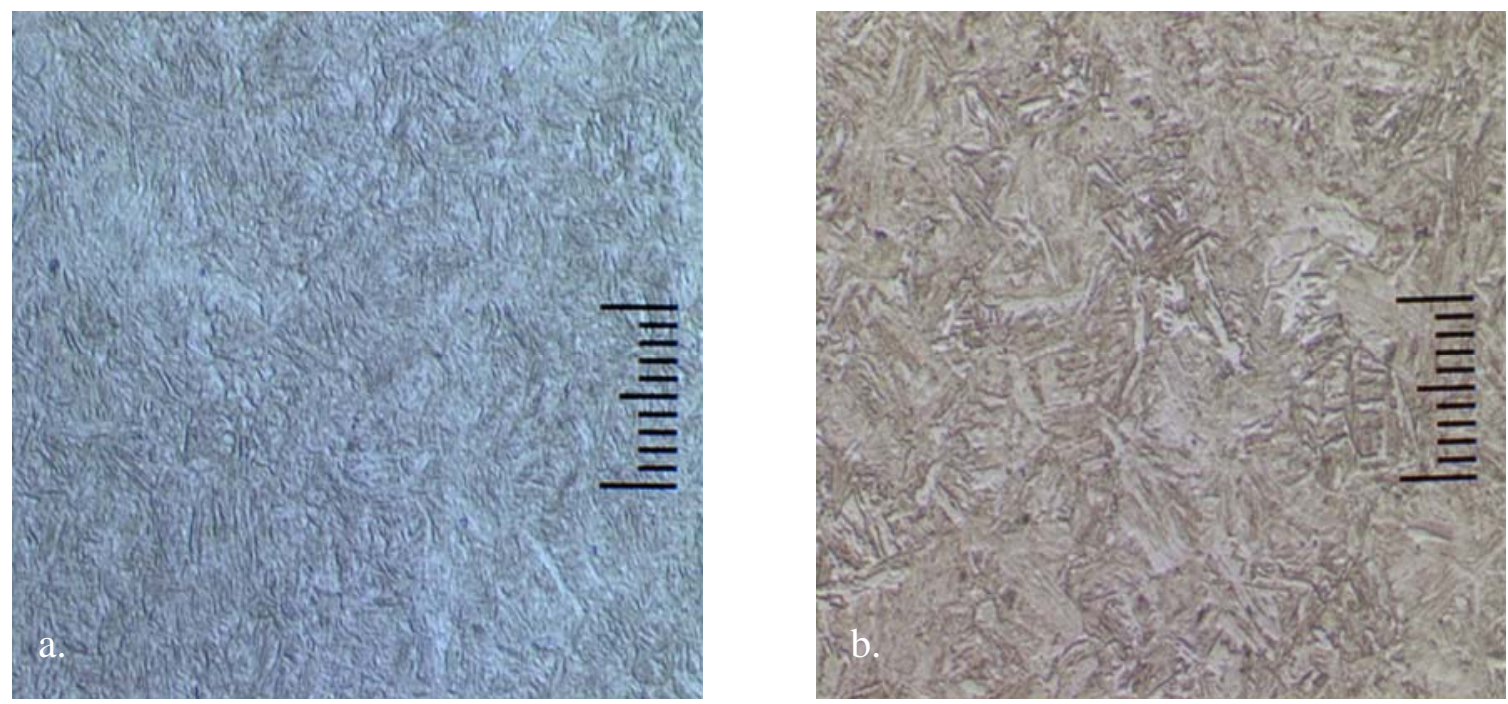

Figure 4-10: Optical images of 4140 microstructures, a. tempered martensite, b. bainite. Scale bar $=20$ micrometers. 


\section{Percent Fracture Strength Vs Hardness (4140)}

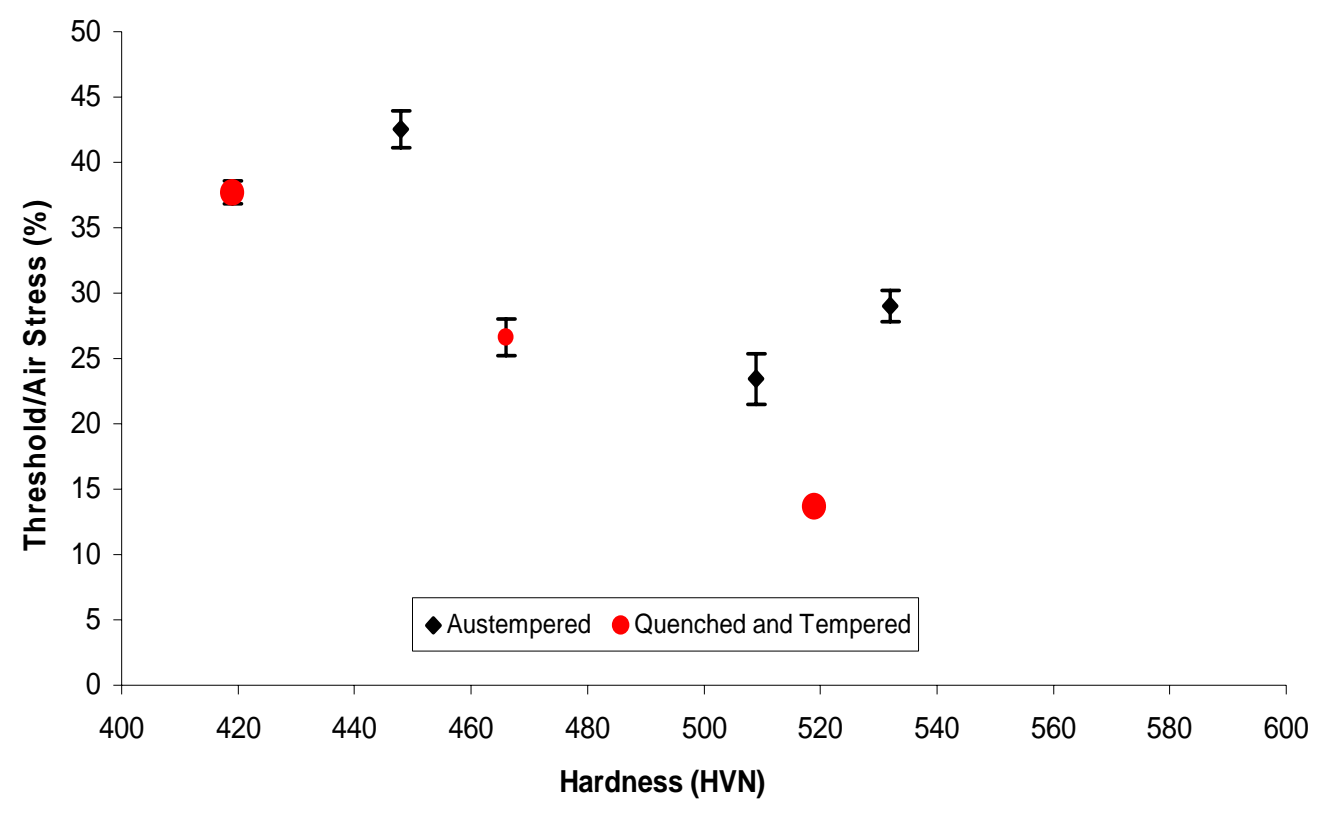

Figure 4-11: Hydrogen-induced cracking susceptibility of 4140 austempered and quenched and tempered steels. 
Percent Fracture Strength Vs. Hardness (Carbon Steels)

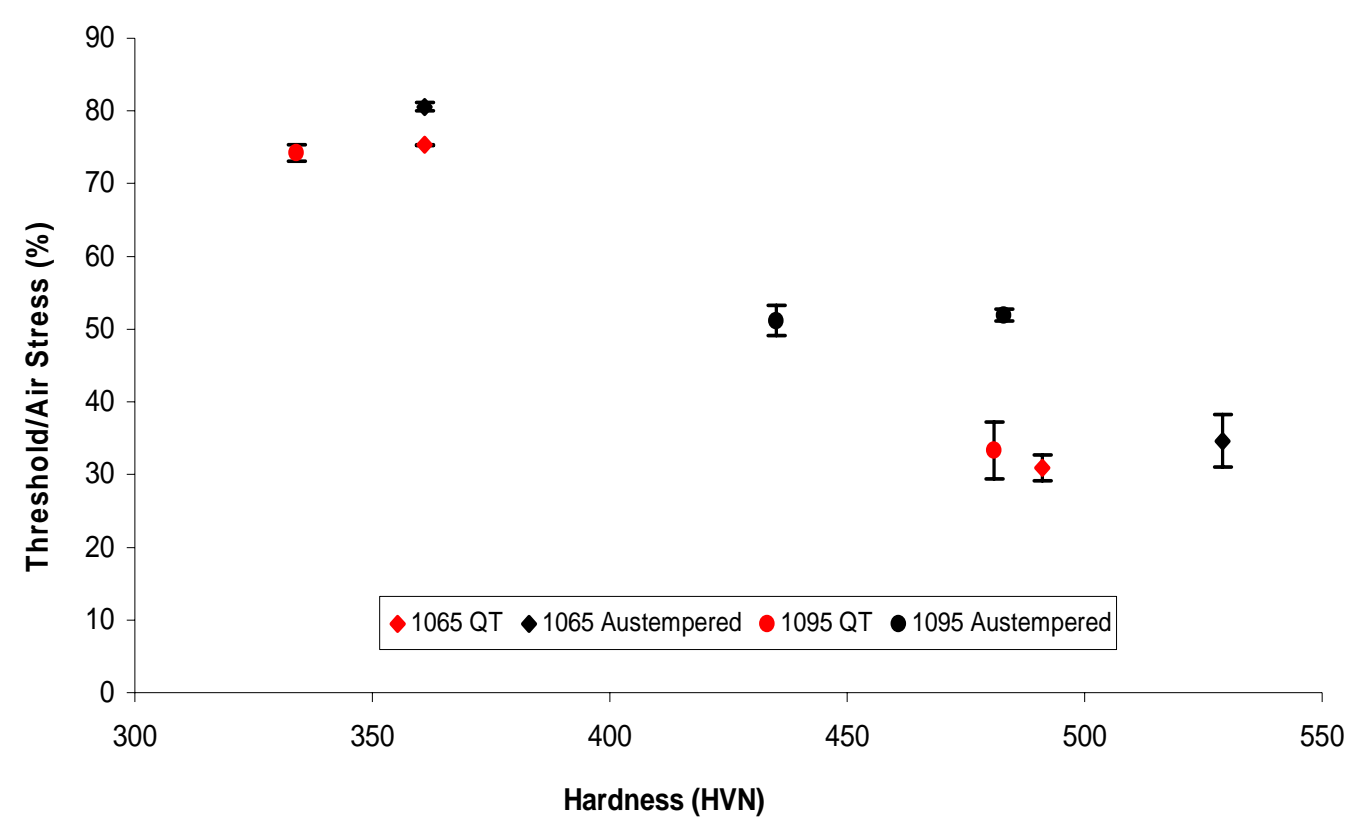

Figure 4-12: Hydrogen-induced cracking sucseptability of 1065 and 1095 austempered and quenched and tempered steel.

The microstructures of the 1095 austempered specimens, at low and intermediate hardnesses, were found to be that of isothermally transformed pearlite and not bainite after high resolution electron microscopy was utilized. Figure 4-13 displays FE-SEM images of the pearlite, as well as the bainite and martensite. In high carbon steels, having carbon contents greater than around $0.6 \mathrm{wt} \%$, only the lower bainitic structure will exist if isothermally treated within the given phase field temperatures, see Figure 2-8. The actual carbon concentrations of the 1065 and 1095 grades of steel are 0.65 and $1.02 \mathrm{wt} \%$, respectively. The temperatures utilized for the 1095 heat treatment were 204, 325 and $384^{\circ} \mathrm{C}$. When examining these temperatures at a carbon concentration of $1.02 \mathrm{wt} \%$, it is obvious that the two higher temperatures lie very near the bainite start temperature, at the transition from pearlite. The images in Figure 4-13, showing pearlitic structures, 
confirms that the temperatures utilized were too high to produce a fully lower bainitic structure. The isothermal temperatures used for the 1065 were 325 and $362^{\circ} \mathrm{C}$, these temperatures fall within the lower bainite region in Figure 2-8. Images of the 1065 structures, displayed in Figure 4-14, verify the existence of lower bainite.
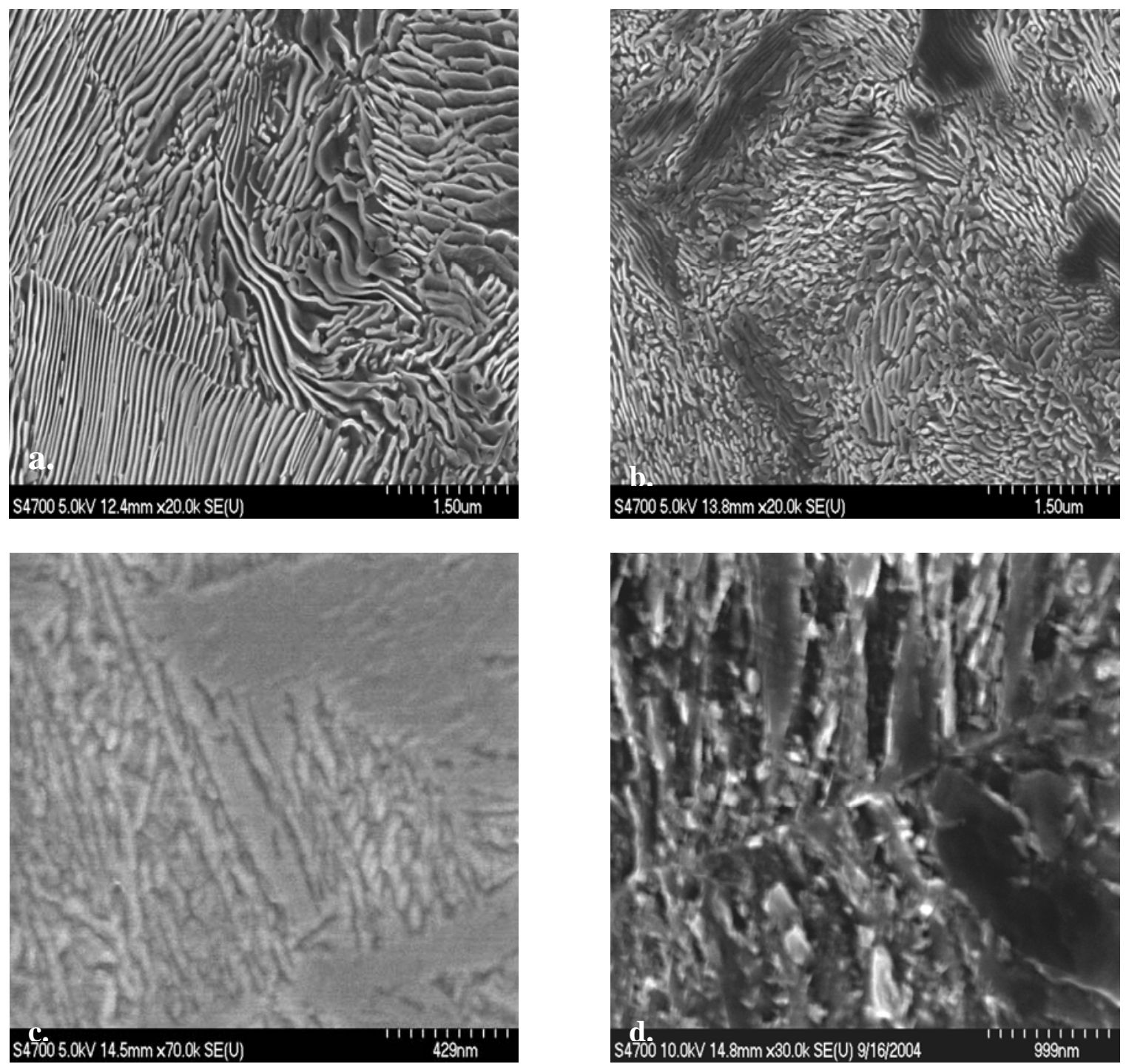

Figure 4-13: SEM images of 1095 isothermally treated steels; a. 1095, $435 \mathrm{HVN}$, fine pearlite, b. 1095, 483 HVN, degenerate pearlite, c. 1095, 776 HVN, lower bainite, d. 4140, 419 HVN, tempered martensite. 

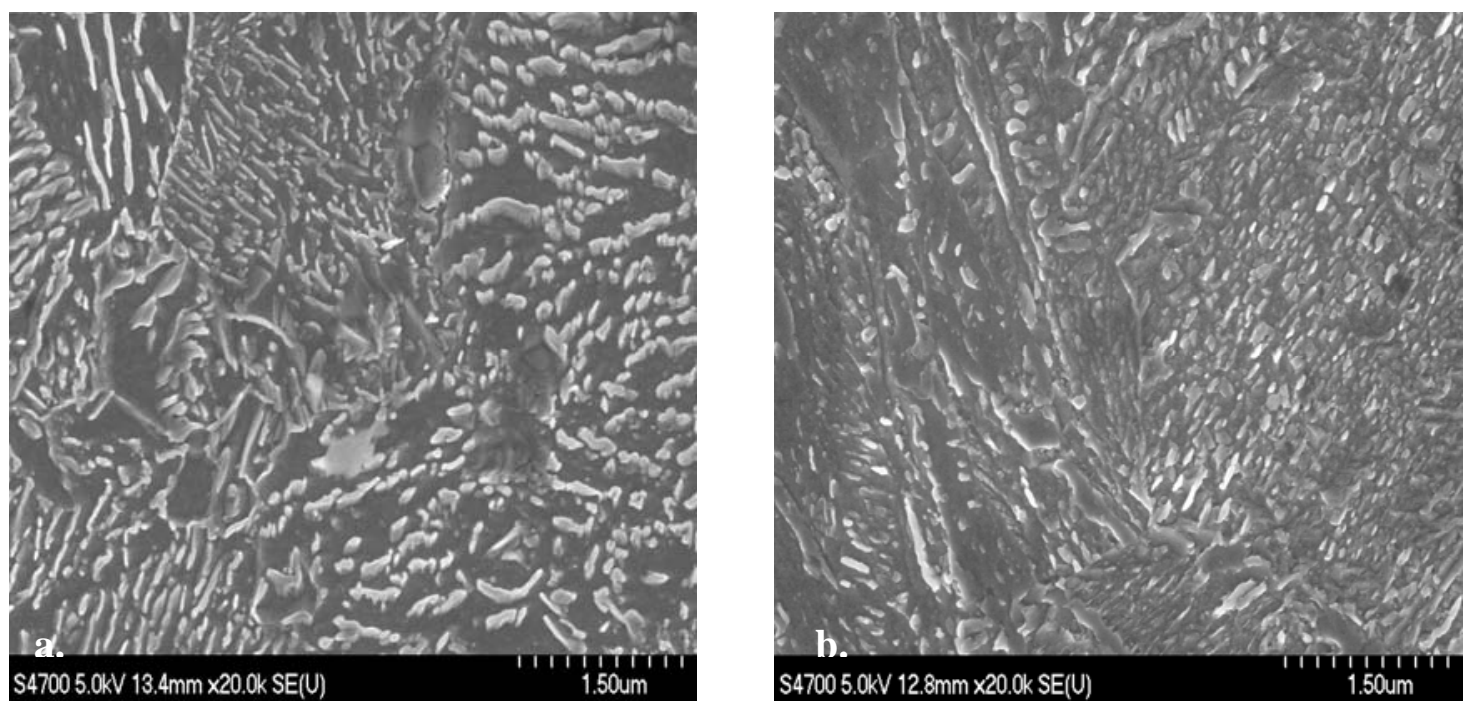

Figure 4-14: SEM images of 1065 microstructures; a. $361 \mathrm{HVN}$, lower bainite, b. $529 \mathrm{HVN}$, lower bainite.

Even though a bainitic structure was desired for the testing, some interesting results were realized when examining the effects of a pearlitic structure on resistance to stress corrosion cracking. Before examining the RSL results, it should be noted that very high hardness values were achieved with the refining of the pearlitic microstructure. The hardness values obtained, 435 and 483, correspond to yield strengths of approximately 1200 and $1350 \mathrm{MPa}$, respectively. The relatively high strengths are attributed to the fine pearlite spacing, measured at around 500 Angstroms. These are much higher than for conventional pearlite isothermal treatments, where yield strengths are around 600-700 Mpa [37], but a bit less than isothermally treated pearlite steels, that are highly cold drawn after forming pearlite, and can have yields around 1600-1700 Mpa [38]. The hydrogen behavior in steels of the lower strength and cold drawn steels has been studied $[37,38,39]$ but little was found in the literature on pearlitic steels where high strength was generated solely through isothermal heat treatments. In one study, it was found that 
1080 pearlite steel, with yield strength of around $600 \mathrm{Mpa}$, maintained its fracture and yield strength when tested in air and hydrogen, but underwent an 82\% loss in RA for the hydrogen charged specimens [39].

The limited RSL data for the high strength isothermal pearlitic steels tested during this research shows a prominent increase in HIC resistance. This is most evident in the high hardness pearlitic steel, shown in figure 4-12. The notched fracture surface of the pearlitic structures in air and hydrogen charging was cleavage and can be seen in figure 4-15. This increased resistance must be attributed to the morphology of the carbide and ferrite, since the relative amount of carbides should be the same for quenched and tempered and isothermally transformed 1095 steels. The plate-like morphology of the finely spaced cementite must inhibit the initiation or growth of cracks that would normally form when charged with hydrogen or possibly limit the diffusion of hydrogen to the location of maximum triaxial stress. High strength, isothermally transformed, structures show very interesting mechanical properties and hydrogen assisted cracking behavior in these limited experiments. It would be beneficial to study these structures in more depth. 

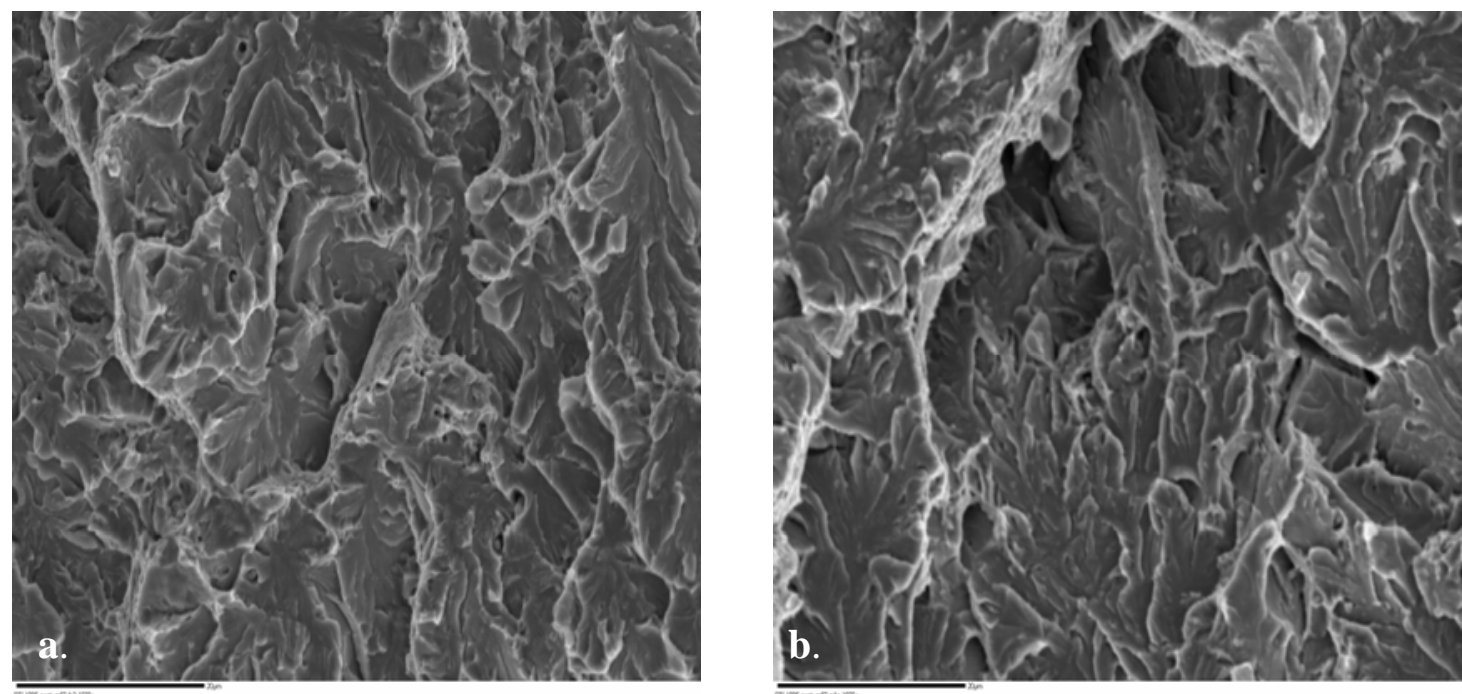

Figure 4-15: 1095 pearlitic steel fracture surfaces; a. air, b. salt solution. (Both images captured at 1500x magnification)

\section{2: Ferrite Crystallite Size Analysis}

The crystallite size analysis of the steels is related to either the sub-ferrite grain sizes and/or the spacing of carbide particles. Estimation of this can be utilized to quantitatively describe differences in microstructures, specifically variations due to tempering. Ferrite crystallite size measurements were taken on all heat treatment 4140, 1065 and 1095 grade steel specimens. A plot of the hardness as a function of the inverse square root of the crystallite size is shown in Figure 4-16. The trend is linear and follows a Hall-Petch relationship. The trend is very well behaved and there are few differences between microstructure and alloy. Due to limited data scatter, it is concluded that the crystallite size does not change much between isothermal and quenched and tempered microstructures. 
Hardness as a Function of Ferrite Crystallite Size

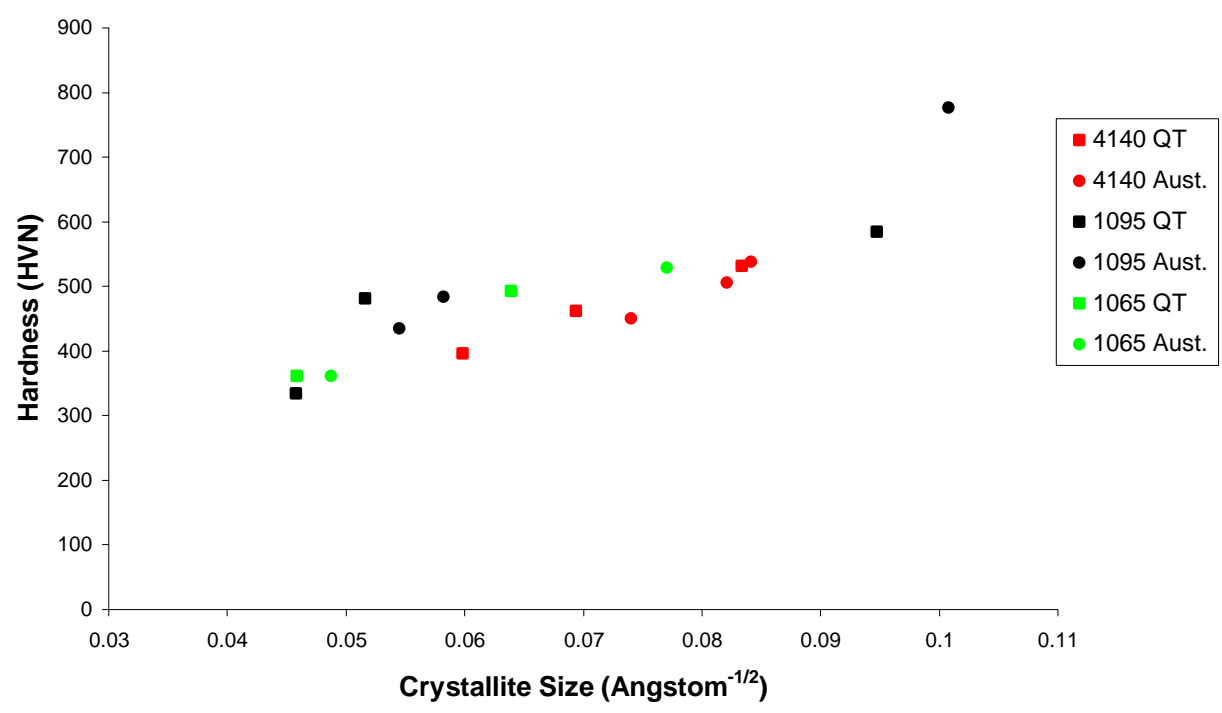

Figure 4-16: Differences in hardness as a function of particle size measurements.

The variation of threshold stress as a percent air fracture strength with the inverse square root of the ferrite crystallite size also produces a linear trend, but the slope is negative. This plot can be viewed in Figure 4-17. As the crystallite size is reduced, the strength of the specimen increases, and the resistance to hydrogen induced cracking decreases. The difference in trends between the isothermal and quenched heat treatments becomes obvious from Figure 4-17. At a given crystallite size, the percentage of threshold stress to air fracture stress is higher for the austempered steels by as much as $20 \%$. This data supplements previous data in verifying an improved resistance of isothermally treated steels. 


\section{Percent Fracture Vs Ferrite Crystallite Size}

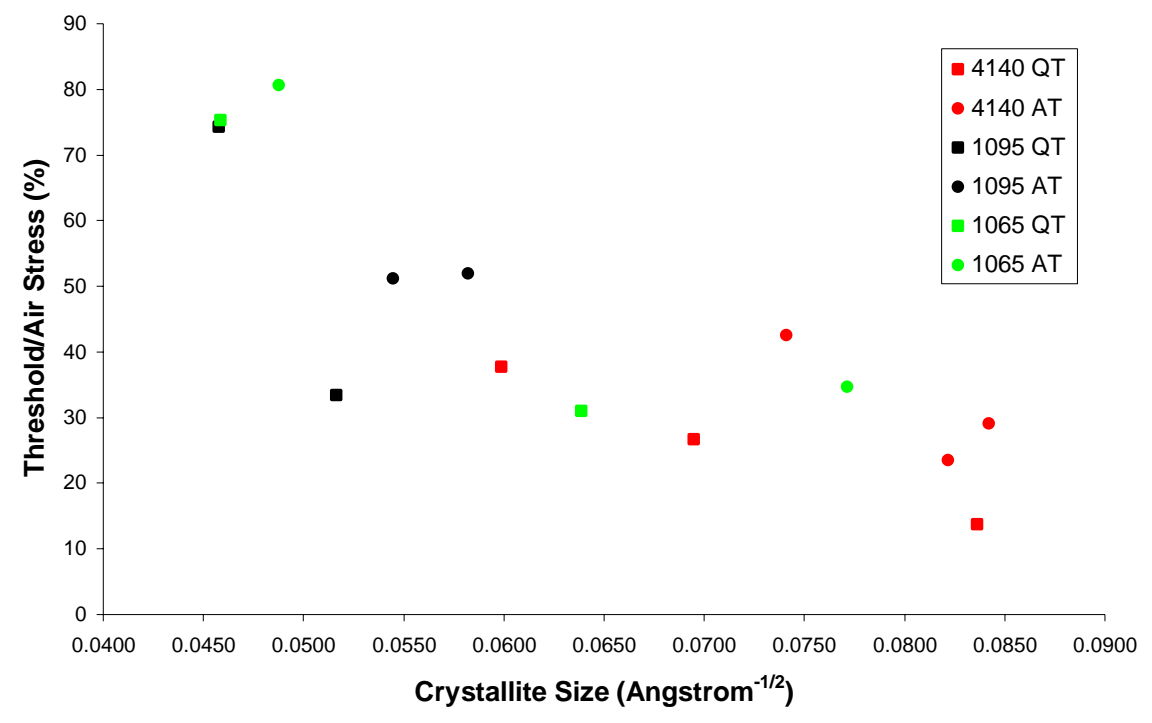

Figure 4-17: Hydrogen-induced cracking susceptibility as a function of the ferrite crystallite size. 


\section{Chapter 5: Conclusions}

1. Dual phase steels consisting of a tempered martensite matrix with islands of 10$20 \%$ by volume ferrite are notch sensitive and less resistant to hydrogen assisted cracking susceptibility than fully tempered martensitic steels, independent of the morphology of the phases resulting from step or continuous annealing treatments.

2. Carbon content does not have an influence on the hydrogen assisted cracking susceptibility when the steel is exposed to a nearly unlimited supply of hydrogen. This is because carbides act as irreversible traps and therefore do not limit hydrogen diffusion when the specimen is hydrogen saturated.

3. Chromium may be detrimental in resistance to hydrogen assisted cracking. All other elements explored; boron, molybdenum and manganese, have little or no effect.

4. Bainitic structures show a $10-20 \%$ increase in resistance to hydrogen assisted cracking susceptibility when compared to tempered martensite. This improved resistance may be attributed to the carbide and ferrite morphology, retained austenite or homogenous strain free structure.

5. High strength, isothermally transformed, pearlite can be produced with hardness values nearing $500 \mathrm{HVN}$. This high strength is due to the fine lamella spacing of approximately 500 angstroms. These steels show improvement in resistance to hydrogen assisted cracking in preliminary results. The pearlitic structure may require a higher local hydrogen concentration for crack nucleation. This improved resistance must be explained by the morphology of the ferrite and carbide platelets. 


\section{Chapter 6: References}

1. N. N. Petch, "The Lowering of Fracture-Stress due to Surface Adsorption", Philos. Mag., Ser. 8, Vol 1 (No. 4), 1956, p 331-335

2. A. R. Troiano, "The Role of Hydrogen and Other Interstitials in the Mechanical Behavior of Metals, Trans. ASM, Vol 52, 1960, p 54-80

3. C. D. Beachem, "A New Model for Hydrogen Assisted Cracking ("Hydrogen “Embrittlement”)”, Metall. Trans., Vol 3 (No. 2), 1972, p 437-451

4. J.E. Grochowski, MS Thesis - Michigan Technological University, 2005

5. L. Raymond, "The Susceptibility of Fasteners to Hydrogen Embrittlement and Stress Corrosion Cracking”, Chapter 39 from The Handbook of Bolts and Bolted Joints, by J.H. Bickford \& S. Nassar, Editors, Marcel Dekker, Inc., Publisher

6. R. C. Cippolla, "Stress Intensity Factor Approximations for Cracks Located at the Thread Root Region of Fasteners", Structural Integrity of Fasteners, edited by P. M. Toor, ASTM, 1995, p 109-125

7. P. M. Toor, "An Overview of Structural Integrity of Fasteners", Structural Integrity of Fasteners, edited by P. M. Toor, ASTM, 1995, p ix - xxii

8. Peterson's Stress Concentration Factors, edited by W.D. Pilkey, John Wiley \& Sons, Inc., 1997

9. N. E. Dowling, Mechanical Behavior of Materials, Prentice-Hall, 1993

10. P.F. Timmins, Solutions to Hydrogen Attack in Steels, ASM International, 1997

11. E. A. Steigerwald, F. W. Schaller, A.R. Troiano, "The Role of Stress in Hydrogen Induced Delayed Failure”, Trans. AIME, Vol 218, 1960, p 832 - 841

12. K. Kitajima, "Modelling of Hydrogen-induced fracture in iron”, Materials Science and Engineering A, Vol 176, 1994, 249-253

13. M. Nagumo, "Function of Hydrogen in Embrittlement of High-strength Steels", ISIJ International, Vol 41 (No. 6), 2001, p 590-598

14. N. N. Kinaev, D. R. Cousens, A. Atrens, “Crack Tip Strain Field of AISI 4340”, J. Mater. Sci., Vol 34, 1999, p 4909-4936

15. "F 606 Test Methods for Determining The Mechanical Properties of Externally and Internally Threaded Fasteners, Washers, and Rivets", Annual Book of ASTM Standards, Vol 01.08, 2000, p 211-222 
16. E. D. McCarty, D. Wetzel, B. S. Kloberdanz, "Hydrogen Embrittlement in Automotive Fastener Applications”, SAE International, Technical Paper Series, No. 960312, 1996

17. L. Raymond, “Evaluation of Hydrogen Embrittlement”, ASM Handbook $9^{\text {th }}$ edition, Corrosion Vol 13, 1987, p 283-290

18. "F 1624 Standard Test Method for Measurement of Hydrogen Embrittlement in Steel by the Incremental Loading Technique”, Annual Book of ASTM Standards, Vol 15.03, 2000

19. A. Bayram, A. Uguz, M, Ula, "Effects of Microstructure and Notches on the Mechanical Properties of Dual-Phase Steels”, Materials Characterization (USA). Vol 43 (No. 4), 1999, p 259-269

20. E. Ahmad, T. Manzoor, K. Liaqat, J. I. Akhter, "Effect of Microvoid Formation on the Tensile Properties of Dual-Phase Steel”, Journal of Materials Engineering and Performance, Vol 9 (No. 3), 2000, p 306-310

21. R. Kerr, F. Solana, I. M. Bernstein, A. W. Thompson, "Microstructural Effects on the Stress Corrosion Cracking Behavior of Medium and High Strength Steels”, Met. Trans. A., Vol 18A, 1987, p 1011-1022

22. “Austempering of Steel”, ASM Handbook $9^{\text {th }}$ edition, Vol 4 Heat Treating, 1987, $p$ 104-116

23. H. K. D. H. Bhadesia, Bainite in Steels $2^{\text {nd }}$ edition, IOM Communications Ltd, 2001

24. E. D. McCarthy, G. A. Shulke, "The Effects of Heat Treatment on the Hydrogen Assisted Cracking Susceptibility of Case Hardened Fasteners”, $20^{\text {th }}$ ASM Heat Treating Society Conference Proceedings, Oct. 2000, ASM International, 2000

25. G. Echaniz, T. E. Perez, C. Pampillo, R. C. Newman, R. P. M. Procter, G. W. Lorimer, "The Effect of Microstructure on Hydrogen-Induced Stress Corrosion Cracking of Quenched and Tempered Steels”, Hydrogen Effects in Materials, The Minerals, Metals \& Materials Society, 1996, p 669-677

26. R. Valentini, A. Solina, "Influence of Microstructure on Hydrogen Embrittlement Behaviour of 2-25Cr-1Mo steel”, Mater. Sci. and Tech., Vol 10, 1994, 908-914

27. E. Quadrini, "Study of the Effect of Heat Treatment on Hydrogen Embrittlement of AISI 4340 steel”, J. Mater. Sci., Vol 24 (No. 3), 1989, p 915-920

28. A. W. Thompson, I. M. Bernstein, “Microstructure and Hydrogen Embrittlement”, in Hydrogen Effects in Metals, AIME, 1981, p 291-308 
29. R. Krishnamurthy, C. N. Marzinsky, R. P. Gangloff, "Microstructure and Yield Strength Effects on Hydrogen Environment Fatigue of Steels”, Hydrogen Effects on Material Behavior, The Minerals, Metals \& Materials Society, 1990, p 891-908

30. L. Tau, S. L. I. Chan, C. S. Shin, "Hydrogen Enhanced Fatigue Crack Propagation of Bainitic and Tempered Martensitic Steels”, Corrosion Science (USA), Vol 38 (No. 11), 1996, p 2049-2060

31. I. M. Bernstein, G. M. Pressouyre, “The Role of Traps in the Microstructural Control of Hydrogen Embrittlement of Steels”, Hydrogen Degradation of Ferrous Alloys, Noyes Publications, 1985, p 641-681

32. P. Bruzzoni, G. Domizzi, M. I. Luppo, D. Zalcman, J. Ovejero-Garcia, "Effect of Boron as a Micro-Alloying Element on the Behaviour of a 1038 Steel in a Hydrogen Environment”, Hydrogen Effects in Materials, The Minerals, Metals \& Materials Society, 1996, p 1,001-1,009

33. B. D. Cullity, S. R. Stock, Elements of X-ray Diffraction $3^{\text {rd }}$ edition, Prentice-Hall, Inc., 2001

34. "F 519 Standard Test Method for Mechanical Hydrogen Embrittlement Evaluation of Plating Processes and Service Environments”, Annual Book of ASTM Standards, Vol 15.03, 2000, p 843-854

35. J. M. Capus, “The Mechanism of Temper Brittleness”, Temper Embrittlement in Steel, ASTM, 1968, p 3-19

36. ASM Handbook $9^{\text {th }}$ edition, Vol 4, Heat Treating, 1987, p 84

37. J. Toribio, E. Vasseur, "Hydrogen-Assisted Micro-Damage Evolution in Pearlitic Steel”, J. of Mater. Sci. letters, Vol 16, 1997, p 1345-1348

38. D. G. Enos, J. R. Scully, “A Critical-Strain Criterion for Hydrogen Embrittlement of Cold-Drawn, Ultrafine Pearlitic Steel”, Met. and Mater. Trans. A, Vol 33A, 2002, p 1151-1166

39. J. E. Costa, A. W. Thompson, "Hydrogen Cracking in Nominally Pearlitic 1045 Steel”, Met. Trans. A, Vol 13A, 1982, p 1315-1318 


\section{Appendices}

I. RSL Load Data

II. RSL Stress Calculations

III. X-Ray Diffraction Data

IV. Compilation of Fractographs 


\section{RSL Load Data}

\begin{tabular}{|c|c|c|c|c|c|}
\hline \multirow{2}{*}{ Test } & \multicolumn{5}{|c|}{ Quenched and Tempered } \\
\hline & 10B21 HRC 40 & $\begin{array}{c}1541 \text { HRC } \\
45\end{array}$ & $\begin{array}{c}1541 \text { HRC } \\
35\end{array}$ & $\begin{array}{c}1038 \text { HRC } \\
35\end{array}$ & $\begin{array}{c}1038 \text { HRC } \\
45\end{array}$ \\
\hline Hardness (HVN) & 400 & 460 & 366 & 347 & 492 \\
\hline Hardness (HRC) & 41 & 45 & 37 & 36 & 49 \\
\hline Fracture 1 & 7754 & 8421 & 7037 & 6166 & 7273 \\
\hline Fracture 2 & 7751 & 8276 & 7198 & 6127 & 7209 \\
\hline Fracture 3 & 7729 & & & & 7266 \\
\hline Fracture Average & 7745 & 8349 & 7118 & 6147 & 7249 \\
\hline Standard Deviation & 14 & 103 & 114 & 28 & 35 \\
\hline RSL Air & 7732 & 7905 & 6632 & 6024 & 7176 \\
\hline Pi1 a & 4533 & 4143 & 4982 & 4592 & 2162 \\
\hline Pi1 b & 5429 & 3312 & 5338 & 4912 & 2207 \\
\hline Pi1 c & 5039 & 3317 & 4982 & & 2530 \\
\hline Pi1 Average & 5000 & 3591 & 5101 & 4752 & 2300 \\
\hline Standard Deviation & 449 & 478 & 206 & 226 & 201 \\
\hline $\mathrm{Pi} 2 \mathrm{a}$ & 5250 & 3317 & 4590 & 4797 & 1495 \\
\hline $\mathrm{Pi} 2 \mathrm{~b}$ & 4499 & 3411 & 4844 & 4784 & 2341 \\
\hline $\mathrm{Pi} 2 \mathrm{c}$ & 4751 & 3410 & 4851 & 4567 & 2086 \\
\hline Pi2 Average & 4833 & 3379 & 4762 & 4716 & 1974 \\
\hline Standard Deviation & 382 & 54 & 149 & 129 & 434 \\
\hline $\mathrm{Pi} 3 \mathrm{a}$ & 5074 & 3707 & 5238 & & \\
\hline $\mathrm{Pi} 3 \mathrm{~b}$ & 5314 & 3356 & 4761 & & \\
\hline Pi3 c & 3361 & 3179 & 4762 & & \\
\hline Pi3 Average & 4583 & 3414 & 4920 & & \\
\hline Standard Deviation & 1065 & 269 & 275 & & \\
\hline
\end{tabular}




\section{RSL Load Data}

\begin{tabular}{|c|c|c|c|c|c|}
\hline \multirow{2}{*}{ Test } & \multicolumn{5}{|c|}{ Quenched and Tempered } \\
\hline & 4140 HRC 50 & 4140 HRC 45 & 4140 HRC 40 & 1095 HRC 35 & 1095 HRC 45 \\
\hline Hardness (HVN) & 519 & 466 & 419 & 334 & 481 \\
\hline Hardness (HRC) & 50 & 47 & 42 & 34 & 48 \\
\hline Fracture 1 & 8781 & 7962 & 7404 & 5955 & 7130 \\
\hline Fracture 2 & 8525 & 7850 & 7371 & 5893 & 7727 \\
\hline Fracture 3 & & & & 5365 & 6667 \\
\hline Fracture Average & 8653 & 7906 & 7388 & 5738 & 7175 \\
\hline Standard Deviation & 181 & 79 & 23 & 324 & 531 \\
\hline RSL Air & 8225 & 7576 & 6955 & 5694 & 6455 \\
\hline $\mathrm{Pi} 1 \mathrm{a}$ & 2015 & 2767 & 3323 & 4796 & 2504 \\
\hline Pi1 b & 1533 & 2766 & 3310 & 4578 & 2893 \\
\hline Pi1 c & 1709 & 2767 & 3300 & 4577 & 1783 \\
\hline Pi1 Average & 1752 & 2767 & 3311 & 4650 & 2393 \\
\hline Standard Deviation & 244 & 1 & 12 & 126 & 563 \\
\hline $\mathrm{Pi} 2 \mathrm{a}$ & 1136 & 3042 & 2796 & 4179 & 2774 \\
\hline $\mathrm{Pi} 2 \mathrm{~b}$ & 1192 & 2076 & 2649 & 4412 & 2526 \\
\hline $\mathrm{Pi} 2 \mathrm{c}$ & 1226 & 1927 & 2914 & 4184 & 3501 \\
\hline Pi2 Average & 1185 & 2348 & 2786 & 4258 & 2934 \\
\hline Standard Deviation & 45 & 605 & 133 & 133 & 507 \\
\hline $\mathrm{Pi} 3 \mathrm{a}$ & 1300 & 2358 & 3091 & 4466 & 2965 \\
\hline $\mathrm{Pi} 3 \mathrm{~b}$ & & 1979 & 3083 & 4720 & 1915 \\
\hline $\mathrm{Pi} 3 \mathrm{c}$ & & 1977 & 2674 & 4688 & 4087 \\
\hline Pi3 Average & 1300 & 2105 & 2949 & 4625 & 2989 \\
\hline Standard Deviation & & 219 & 238 & 138 & 1086 \\
\hline
\end{tabular}




\section{RSL Load Data}

\begin{tabular}{|c|c|c|c|}
\hline \multirow{2}{*}{ Test } & \multicolumn{3}{|c|}{ Quenched and Tempered } \\
\hline & 1095 HRC 60 & 1065 HRC 35 & 1065 HRC 55 \\
\hline Hardness (HVN) & 584 & 361 & 491 \\
\hline Hardness (HRC) & 54 & 37 & 49 \\
\hline Fracture 1 & 4322 & 6399 & 7688 \\
\hline Fracture 2 & 4989 & 6617 & 7228 \\
\hline \multicolumn{4}{|l|}{ Fracture 3} \\
\hline Fracture Average & 4656 & 6508 & 7458 \\
\hline Standard Deviation & 472 & 154 & 325 \\
\hline RSL Air & & 6110 & 7088 \\
\hline Pi1 a & 4824 & 4907 & 2610 \\
\hline Pi1 b & 458 & 4893 & 2611 \\
\hline Pi1 c & 1361 & 4910 & 2610 \\
\hline Pi1 Average & 2214 & 4903 & 2610 \\
\hline Standard Deviation & 2305 & 9 & 1 \\
\hline $\mathrm{Pi} 2 \mathrm{a}$ & & 4923 & 2238 \\
\hline $\mathrm{Pi} 2 \mathrm{~b}$ & & 5132 & 2604 \\
\hline $\mathrm{Pi} 2 \mathrm{c}$ & & 5388 & 2089 \\
\hline Pi2 Average & & 5148 & 2310 \\
\hline Standard Deviation & & 233 & 265 \\
\hline \multicolumn{4}{|l|}{$\mathrm{Pi3} \mathrm{a}$} \\
\hline \multicolumn{4}{|l|}{$\mathrm{Pi} 3 \mathrm{~b}$} \\
\hline \multicolumn{4}{|l|}{$\mathrm{Pi3} \mathrm{C}$} \\
\hline \multicolumn{4}{|l|}{ Pi3 Average } \\
\hline Standard Deviation & & & \\
\hline
\end{tabular}




\section{RSL Load Data}

\begin{tabular}{|c|c|c|c|c|c|}
\hline \multirow{2}{*}{ Test } & \multicolumn{5}{|c|}{ Austempered } \\
\hline & 4140 HRC 50 & 4140 HRC 45 & 4140 HRC 40 & 1095 HRC 40 & 1095 HRC 50 \\
\hline Hardness (HVN) & 532 & 509 & 448 & 435 & 483 \\
\hline Hardness (HRC) & 51 & 50 & 45 & 44 & 48 \\
\hline Fracture 1 & 8895 & 8275 & 7805 & 7624 & 8272 \\
\hline Fracture 2 & 8914 & 8300 & 7785 & 7780 & 8430 \\
\hline \multicolumn{6}{|l|}{ Fracture 3} \\
\hline Fracture Average & 8905 & 8288 & 7795 & 7702 & 8351 \\
\hline Standard Deviation & 13 & 18 & 14 & 110 & 112 \\
\hline \multirow[t]{2}{*}{ RSL Air } & 8635 & 8095 & 7310 & 7275 & 7889 \\
\hline & & & & & 4177 \\
\hline $\mathrm{Pi} 1 \mathrm{a}$ & 3319 & 2491 & 4277 & 4554 & 4582 \\
\hline $\mathrm{Pi} 1 \mathrm{~b}$ & 2687 & 2922 & 4709 & 3458 & 4594 \\
\hline $\mathrm{Pi1} \mathrm{c}$ & 3323 & 2917 & 4288 & 4608 & 4171 \\
\hline Pi1 Average & 3110 & 2777 & 4425 & 4207 & 4381 \\
\hline Standard Deviation & 366 & 247 & 246 & 649 & 241 \\
\hline $\mathrm{Pi} 2 \mathrm{a}$ & 2781 & 2218 & 4031 & 4214 & 4431 \\
\hline $\mathrm{Pi} 2 \mathrm{~b}$ & 3356 & 2088 & 3776 & 4029 & 4238 \\
\hline $\mathrm{Pi} 2 \mathrm{c}$ & 3366 & 2727 & 3564 & 3580 & \\
\hline Pi2 Average & 3168 & 2344 & 3790 & 3941 & 4335 \\
\hline Standard Deviation & 335 & 338 & 234 & 326 & 136 \\
\hline $\mathrm{Pi} 3 \mathrm{a}$ & 2746 & 2743 & 2859 & & \\
\hline Pi3 b & 3143 & 2240 & 4189 & & \\
\hline Pi3 c & 3135 & 2477 & 4388 & & \\
\hline Pi3 Average & 3008 & 2487 & 3812 & & \\
\hline Standard Deviation & 227 & 252 & 831 & & \\
\hline
\end{tabular}




\section{RSL Load Data}

\begin{tabular}{|c|c|c|c|c|c|}
\hline \multirow{2}{*}{ Test } & \multicolumn{3}{|c|}{ Austempered } & \multicolumn{2}{|c|}{ Dual Phase } \\
\hline & 1095 HRC 60 & 1065 HRC 40 & 1065 HRC 50 & 4037 HRC 35 & 4037 HRC 45 \\
\hline Hardness (HVN) & 776 & 361 & 529 & 389 & 473 \\
\hline Hardness (HRC) & 63 & 37 & 51 & 40 & 47 \\
\hline Fracture 1 & 5348 & 6686 & 9138 & 6644 & 6005 \\
\hline Fracture 2 & 4892 & 6684 & 8757 & 5982 & 6223 \\
\hline Fracture 3 & 5830 & & & 6356 & 6480 \\
\hline Fracture Average & 5357 & 6685 & 8948 & 6327 & 6236 \\
\hline Standard Deviation & 469 & 1 & 269 & 332 & 238 \\
\hline RSL Air & & 6539 & 8430 & 6188 & 5579 \\
\hline $\mathrm{Pi1}$ a & 1341 & 5660 & 3566 & 3500 & 2184 \\
\hline $\mathrm{Pi1} b$ & 1071 & 5648 & 3131 & 4104 & 2511 \\
\hline $\mathrm{Pi1} \mathrm{c}$ & 1604 & 5641 & 2702 & 3476 & 2501 \\
\hline Pi1 Average & 1339 & 5650 & 3133 & 3693 & 2399 \\
\hline Standard Deviation & 267 & 10 & 432 & 356 & 186 \\
\hline $\mathrm{Pi} 2 \mathrm{a}$ & & 5342 & 3472 & 4417 & 1937 \\
\hline $\mathrm{Pi} 2 \mathrm{~b}$ & & 5477 & 3471 & 3680 & 2279 \\
\hline $\mathrm{Pi} 2 \mathrm{C}$ & & 5342 & 2357 & 3873 & 2053 \\
\hline Pi2 Average & & 5387 & 3100 & 3990 & 2090 \\
\hline Standard Deviation & & 78 & 643 & 382 & 174 \\
\hline $\mathrm{Pi} 3 \mathrm{a}$ & & & & 3990 & 1978 \\
\hline $\mathrm{Pi} 3 \mathrm{~b}$ & & & & 3417 & 2611 \\
\hline $\mathrm{Pi} 3 \mathrm{C}$ & & & & 3777 & 1672 \\
\hline Pi3 Average & & & & 3728 & 2087 \\
\hline Standard Deviation & & & & 290 & 479 \\
\hline
\end{tabular}




\section{RSL Stress Calculations}

\begin{tabular}{|c|c|c|c|c|c|}
\hline \multirow{2}{*}{ Test } & \multicolumn{5}{|c|}{ Quenched and Tempered } \\
\hline & 10B21 HRC 40 & 1541 HRC 35 & 1541 HRC 45 & 1038 HRC 35 & 1038 HRC 45 \\
\hline Vickers Hardness & 400 & 366 & 460 & 347 & 492 \\
\hline \multicolumn{6}{|l|}{ Air Testing } \\
\hline Air Load (Ibs) & 7745 & 7118 & 8349 & 6147 & 7249 \\
\hline Air Stress (ksi) & 322 & 296 & 347 & 256 & 301 \\
\hline Max Air Load (lbs) & 7754 & 7198 & 8421 & 6166 & 7273 \\
\hline Maximum Air (ksi) & 322 & 299 & 350 & 256 & 302 \\
\hline Dev. & 0 & 3 & 3 & 1 & 1 \\
\hline Min Air Load (lbs) & 7729 & 7037 & 8276 & 6127 & 7209 \\
\hline Minimum Air (ksi) & 321 & 293 & 344 & 255 & 300 \\
\hline Dev. & 1 & 3 & 3 & 1 & 2 \\
\hline Deviation in Air (lbs) & 14 & 114 & 103 & 28 & 35 \\
\hline Std. Dev. (ksi) & 1 & 5 & 4 & 1 & 1 \\
\hline Salt Testing/Protocol & 3 & 2 & 2 & 2 & 2 \\
\hline Threshold Load (lbs) & 4583 & 4762 & 3379 & 4716 & 1974 \\
\hline Threshold Stress (ksi) & 191 & 198 & 140 & 196 & 82 \\
\hline Max Load (lbs) & 5314 & 4851 & 3411 & 4797 & 2341 \\
\hline Maximum (ksi) & 221 & 202 & 142 & 199 & 97 \\
\hline Min Load (lbs) & 3361 & 4590 & 3317 & 4567 & 1495 \\
\hline Minimum (ksi) & 140 & 191 & 138 & 190 & 62 \\
\hline Deviation of Load (lbs) & 1065 & 149 & 54 & 129 & 434 \\
\hline $\begin{array}{l}\text { Standard Deviation } \\
\text { (ksi) }\end{array}$ & 44 & 6 & 2 & 5 & 18 \\
\hline Percent FS (\%) & 59 & 67 & 40 & 77 & 27 \\
\hline
\end{tabular}




\section{RSL Stress Calculations}

\begin{tabular}{|c|c|c|c|c|c|}
\hline \multirow{2}{*}{ Test } & \multicolumn{5}{|c|}{ Quenched and Tempered } \\
\hline & 4140 HRC 40 & 4140 HRC 45 & 4140 HRC 50 & 1095 HRC 35 & 1095 HRC 45 \\
\hline Vickers Hardness & 419 & 466 & 519 & 334 & 481 \\
\hline \multicolumn{6}{|l|}{ Air Testing } \\
\hline Air Load (Ibs) & 7388 & 7906 & 8653 & 5738 & 7175 \\
\hline Air Stress (ksi) & 307 & 329 & 360 & 239 & 298 \\
\hline Max Air Load (lbs) & 7404 & 7962 & 8781 & 5955 & 7727 \\
\hline Maximum Air (ksi) & 308 & 331 & 365 & 248 & 321 \\
\hline Dev. & 1 & 2 & 5 & 9 & 23 \\
\hline Min Air Load (lbs) & 7371 & 7850 & 8525 & 5365 & 6667 \\
\hline Minimum Air (ksi) & 306 & 326 & 354 & 223 & 277 \\
\hline Dev. & 1 & 2 & 5 & 16 & 21 \\
\hline Deviation in Air (lbs) & 23 & 79 & 181 & 324 & 531 \\
\hline Std. Dev. (ksi) & 1 & 3 & 8 & 13 & 22 \\
\hline Salt Testing/Protocol & 2 & 3 & 2 & 2 & 1 \\
\hline Threshold Load (lbs) & 2786 & 2105 & 1185 & 4258 & 2393 \\
\hline Threshold Stress (ksi) & 116 & 88 & 49 & 177 & 99 \\
\hline Max Load (lbs) & 2914 & 2358 & 1226 & 4412 & 2893 \\
\hline Maximum (ksi) & 121 & 98 & 51 & 183 & 120 \\
\hline Min Load (lbs) & 2649 & 1979 & 1136 & 4184 & 1783 \\
\hline Minimum (ksi) & 110 & 82 & 47 & 174 & 74 \\
\hline Deviation of Load (lbs) & 133 & 219 & 45 & 133 & 563 \\
\hline $\begin{array}{l}\text { Standard Deviation } \\
\text { (ksi) }\end{array}$ & 6 & 9 & 2 & 6 & 23 \\
\hline Percent FS (\%) & 38 & 27 & 14 & 74 & 33 \\
\hline
\end{tabular}




\section{RSL Stress Calculations}

\begin{tabular}{|c|c|c|c|c|}
\hline \multirow[t]{2}{*}{ Test } & \multicolumn{2}{|c|}{$\begin{array}{l}\text { Quenched and } \\
\text { Tempered }\end{array}$} & \multicolumn{2}{|c|}{ Dual Phase } \\
\hline & 1065 HRC 35 & 1065 HRC 55 & 4037 HRC 35 & 4037 HRC 45 \\
\hline Vickers Hardness & 361 & 491 & 389 & 473 \\
\hline \multicolumn{5}{|l|}{ Air Testing } \\
\hline Air Load (Ibs) & 6508 & 7458 & 6327 & 6236 \\
\hline Air Stress (ksi) & 271 & 310 & 263 & 259 \\
\hline Max Air Load (lbs) & 6617 & 7688 & 6644 & 6480 \\
\hline Maximum Air (ksi) & 275 & 320 & 276 & 269 \\
\hline Dev. & 5 & 10 & & \\
\hline Min Air Load (lbs) & 6399 & 7228 & 5982 & 6005 \\
\hline Minimum Air (ksi) & 266 & 301 & 249 & 250 \\
\hline Dev. & 5 & 10 & & \\
\hline Deviation in Air (lbs) & 154 & 325 & 332 & 238 \\
\hline Std. Dev. (ksi) & 6 & 14 & 14 & 10 \\
\hline Salt Testing/Protocol & 1 & 2 & 1 & 3 \\
\hline Threshold Load (Ibs) & 4903 & 2310 & 3693 & 2087 \\
\hline Threshold Stress (ksi) & 204 & 96 & 154 & 87 \\
\hline Max Load (lbs) & 4910 & 2604 & 4104 & 2611 \\
\hline Maximum (ksi) & 204 & 108 & 171 & 109 \\
\hline Min Load (lbs) & 4893 & 2089 & 3476 & 1672 \\
\hline Minimum (ksi) & 203 & 87 & 145 & 70 \\
\hline Deviation of Load (lbs) & 9 & 265 & 356 & 479 \\
\hline $\begin{array}{l}\text { Standard Deviation } \\
\text { (ksi) }\end{array}$ & 0 & 11 & 15 & 20 \\
\hline Percent FS (\%) & 75 & 31 & 58 & 33 \\
\hline
\end{tabular}




\section{RSL Stress Calculations}

\begin{tabular}{|c|c|c|c|c|c|}
\hline \multirow{2}{*}{ Test } & \multicolumn{5}{|c|}{ Austempered } \\
\hline & 4140 HRC 40 & 4140 HRC 45 & 4140 HRC 50 & 1095 HRC 40 & 1095 HRC 50 \\
\hline Vickers Hardness & 448 & 509 & 532 & 435 & 483 \\
\hline \multicolumn{6}{|l|}{ Air Testing } \\
\hline Air Load (lbs) & 8166 & 8659 & 9276 & 7702 & 8351 \\
\hline Air Stress (ksi) & 340 & 360 & 386 & 320 & 347 \\
\hline Max Air Load (lbs) & 8176 & 8671 & 9285 & 7780 & 8430 \\
\hline Maximum Air (ksi) & 340 & 360 & 386 & 323 & 350 \\
\hline Dev. & 0 & 0 & 0 & 3 & 3 \\
\hline Min Air Load (lbs) & 8156 & 8646 & 9266 & 7624 & 8272 \\
\hline Minimum Air (ksi) & 339 & 359 & 385 & 317 & 344 \\
\hline Dev. & 0 & 1 & 0 & 3 & 3 \\
\hline Deviation in Air (lbs) & 14 & 18 & 13 & 110 & 112 \\
\hline Std. Dev. (ksi) & 1 & 1 & 1 & 5 & 5 \\
\hline Salt Testing/Protocol & 2 & 2 & 3 & 2 & 2 \\
\hline Threshold Load (Ibs) & 3474 & 2028 & 2692 & 3941 & 4335 \\
\hline Threshold Stress (ksi) & 144 & 84 & 112 & 164 & 180 \\
\hline Max Load (Ibs) & 3715 & 2411 & 2827 & 4214 & 4431 \\
\hline Maximum (ksi) & 154 & 100 & 118 & 175 & 184 \\
\hline Min Load (Ibs) & 3247 & 1771 & 2429 & 3580 & 4238 \\
\hline Minimum (ksi) & 135 & 74 & 101 & 149 & 176 \\
\hline Deviation of Load (Ibs) & 234 & 338 & 227 & 326 & 136 \\
\hline $\begin{array}{l}\text { Standard Deviation } \\
\text { (ksi) }\end{array}$ & 10 & 14 & 9 & 14 & 6 \\
\hline Percent FS (\%) & 43 & 23 & 29 & 51 & 52 \\
\hline
\end{tabular}




\section{RSL Stress Calculations}

\begin{tabular}{|c|c|c|}
\hline \multirow{2}{*}{ Test } & \multicolumn{2}{|c|}{ Austempered } \\
\hline & 1065 HRC 40 & 1065 HRC 50 \\
\hline Vickers Hardness & 361 & 529 \\
\hline \multicolumn{3}{|l|}{ Air Testing } \\
\hline Air Load (lbs) & 6685 & 8948 \\
\hline Air Stress (ksi) & 278 & 372 \\
\hline Max Air Load (lbs) & 6686 & 9138 \\
\hline Maximum Air (ksi) & 278 & 380 \\
\hline Dev. & 0 & 8 \\
\hline Min Air Load (lbs) & 6684 & 8757 \\
\hline Minimum Air (ksi) & 278 & 364 \\
\hline Dev. & 0 & 8 \\
\hline Deviation in Air (lbs) & 1 & 269 \\
\hline Std. Dev. (ksi) & 0 & 11 \\
\hline Salt Testing/Protocol & 2 & 2 \\
\hline Threshold Load (Ibs) & 5387 & 3100 \\
\hline Threshold Stress (ksi) & 224 & 129 \\
\hline Max Load (Ibs) & 5477 & 3472 \\
\hline Maximum (ksi) & 228 & 144 \\
\hline Min Load (Ibs) & 5342 & 2357 \\
\hline Minimum (ksi) & 222 & 98 \\
\hline Deviation of Load (lbs) & 78 & 643 \\
\hline $\begin{array}{l}\text { Standard Deviation } \\
\text { (ksi) }\end{array}$ & 3 & 27 \\
\hline Percent FS (\%) & 81 & 35 \\
\hline
\end{tabular}




\section{X-Ray Diffraction Data}

\begin{tabular}{|c|c|c|c|c|c|c|}
\hline \multicolumn{7}{|c|}{211 Peak Ferrite Peak } \\
\hline Steel Grade & Sample ID & $\begin{array}{l}\text { Hardness } \\
\text { (HVN) }\end{array}$ & $\begin{array}{l}\text { Peak } \\
\text { Position } \\
\text { (deg.) }\end{array}$ & $\begin{array}{l}\text { FWHM } \\
\text { (deg.) }\end{array}$ & $\begin{array}{l}\text { Crystallite Size } \\
\text { (Angstroms) }\end{array}$ & $\begin{array}{c}d^{-1 / 2} \\
\left(A^{-1 / 2}\right)\end{array}$ \\
\hline \multirow{3}{*}{ Q\&T 4140} & Rc50 & 531 & 82.20 & 0.7826 & 144 & 0.0834 \\
\hline & Rc45 & 462 & 82.21 & 0.5588 & 207 & 0.0694 \\
\hline & Rc40 & 396 & 82.20 & 0.4299 & 279 & 0.0599 \\
\hline \multirow{2}{*}{ Q\&T 1065} & Rc40 & 361 & 82.23 & 0.3059 & 420 & 0.0488 \\
\hline & Rc50 & 529 & 82.19 & 0.6760 & 168 & 0.0771 \\
\hline \multirow{3}{*}{ Q\&T 1095} & Rc35 & 334 & 82.27 & 0.2771 & 477 & 0.0458 \\
\hline & Rc45 & 481 & 82.16 & 0.3350 & 375 & 0.0516 \\
\hline & Rc60 & 584 & 82.06 & 0.9943 & 111 & 0.0948 \\
\hline \multirow{3}{*}{ AT 4140} & Rc51 & 538 & 82.20 & 0.7951 & 141 & 0.0841 \\
\hline & Rc50 & 506 & 82.20 & 0.7600 & 148 & 0.0821 \\
\hline & Rc45 & 450 & 82.19 & 0.6278 & 182 & 0.0740 \\
\hline \multirow{2}{*}{ AT 1065} & Rc40 & 361 & 82.23 & 0.3059 & 420 & 0.0488 \\
\hline & Rc50 & 529 & 82.19 & 0.6760 & 168 & 0.0771 \\
\hline \multirow{3}{*}{ AT 1095} & Rc40 & 435 & 82.23 & 0.3666 & 337 & 0.0545 \\
\hline & Rc50 & 483 & 82.25 & 0.4099 & 295 & 0.0582 \\
\hline & Rc60 & 776 & 82.11 & 1.1187 & 98 & 0.1008 \\
\hline
\end{tabular}


IV. Compilation of Fractographs

\section{QT 10B21 - 400 HVN}

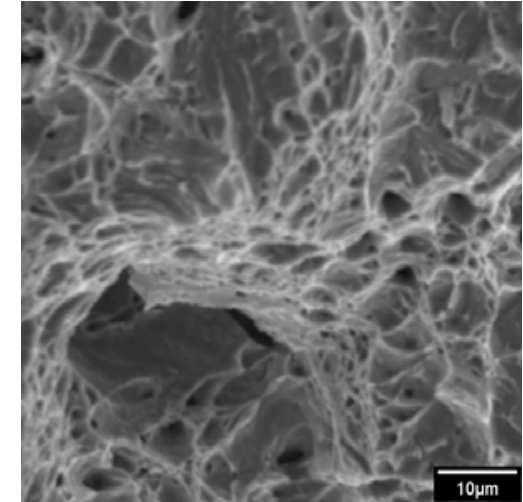

Air - MVC

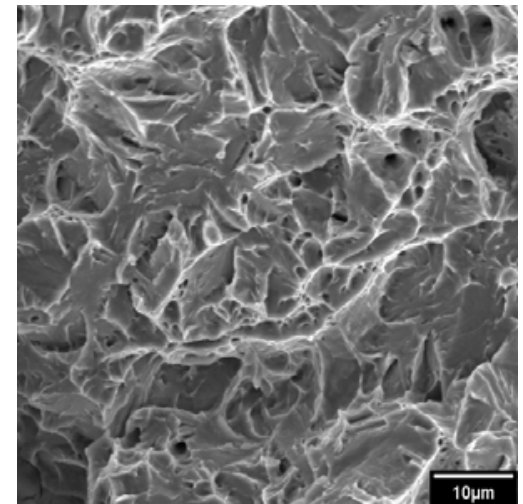

Hydrogen charged - Cleavage

\section{QT 1541 - 366 HVN}

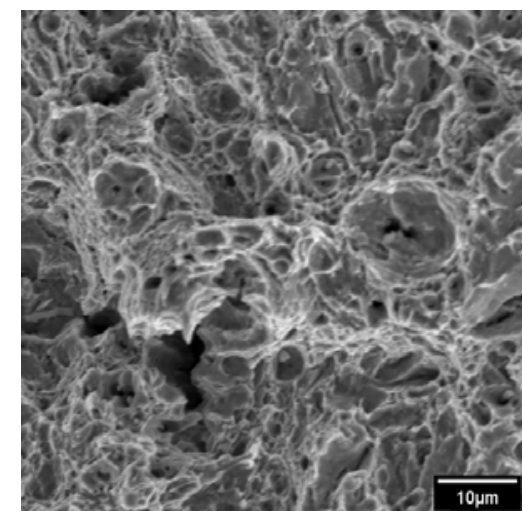

Air - MVC/Quasi-Cleavage

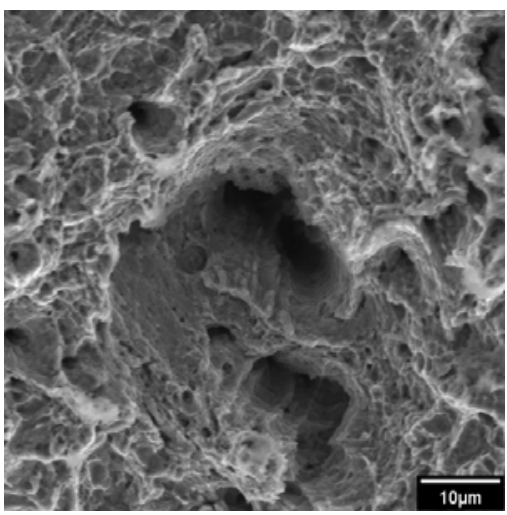

Hydrogen charged - Quasi-Cleavage/MVC

QT 1541 - 460 HVN
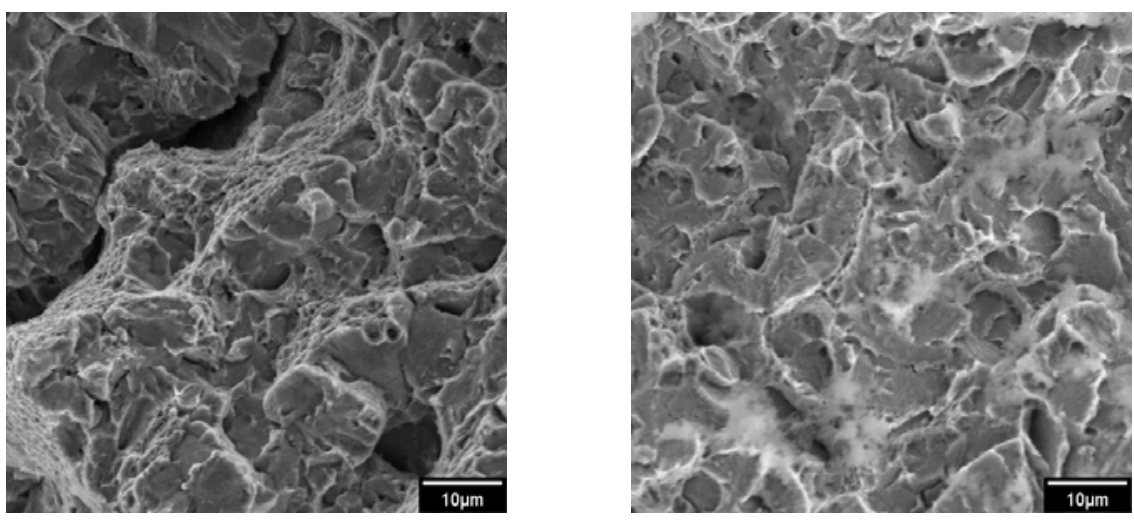


\section{QT 1038 - 347 HVN}

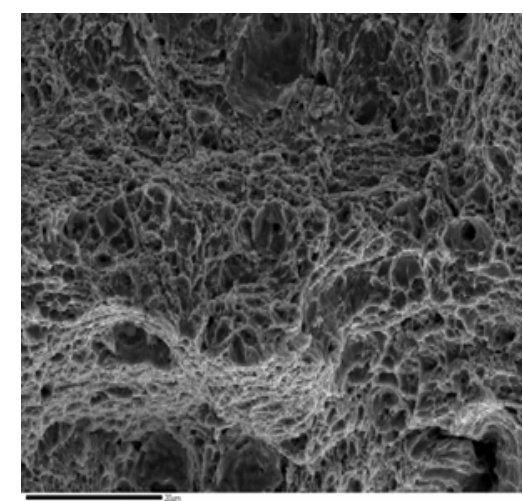

Air - MVC

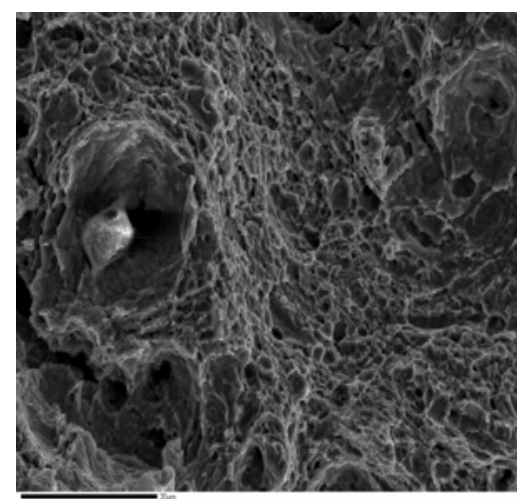

Hydrogen charged - MVC

\section{QT 1038 - 492 HVN}

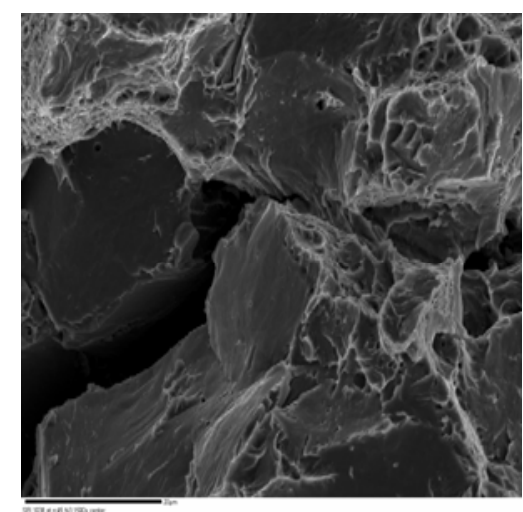

Air - MVC/Cleavage

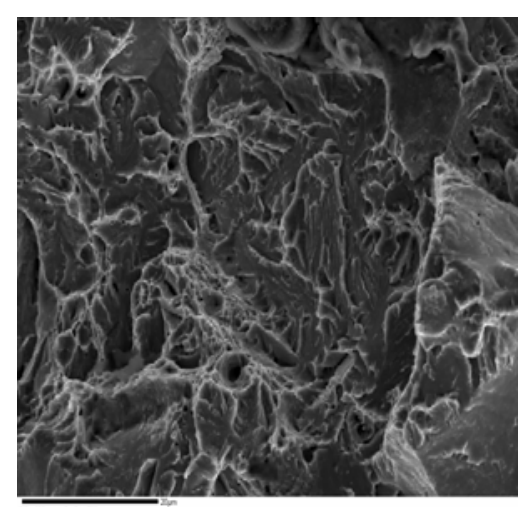

Hydrogen charged - Quasi-Cleavage

\section{QT 4140 - 419, HVN}
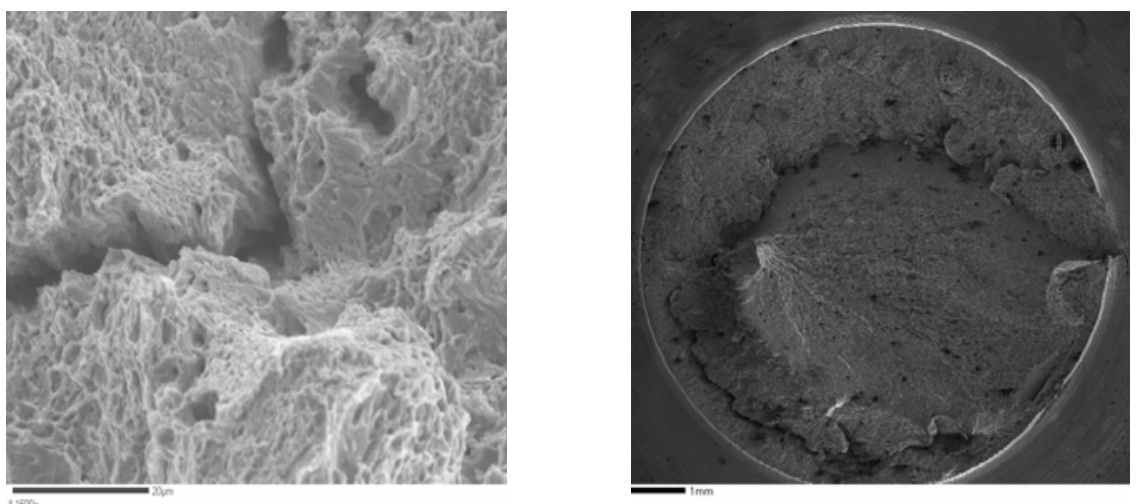
Air - MVC/Micro-cracking

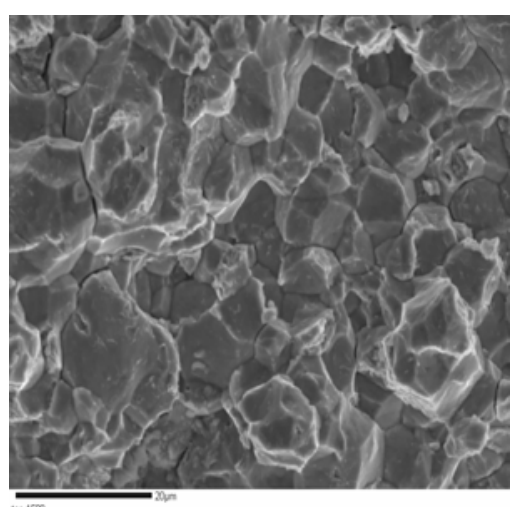

Charged Edge - Intergranular
Hydrogen charged - Low Mag.

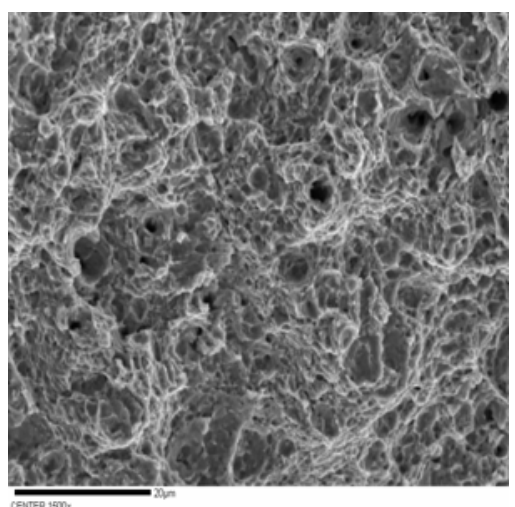

Charged Center - MVC

\section{QT 4140 - 519 HVN (466 HVN And AT are Similar)}

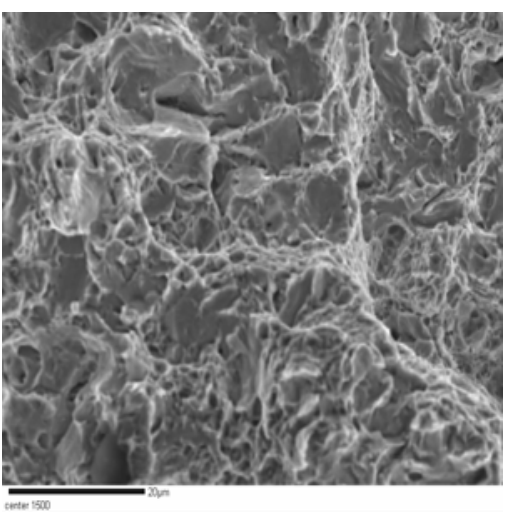

Air - Quasi-Cleavage

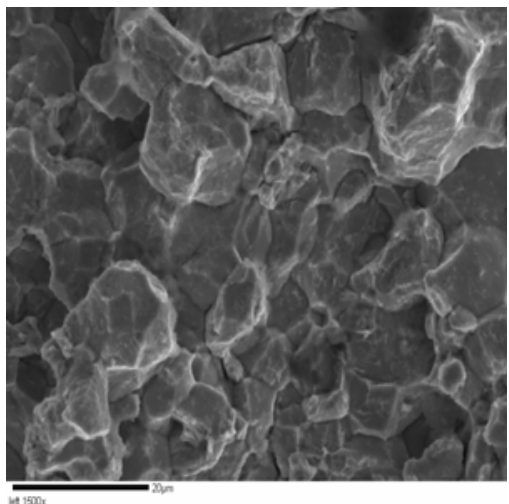

Charged Edge - Intergranular

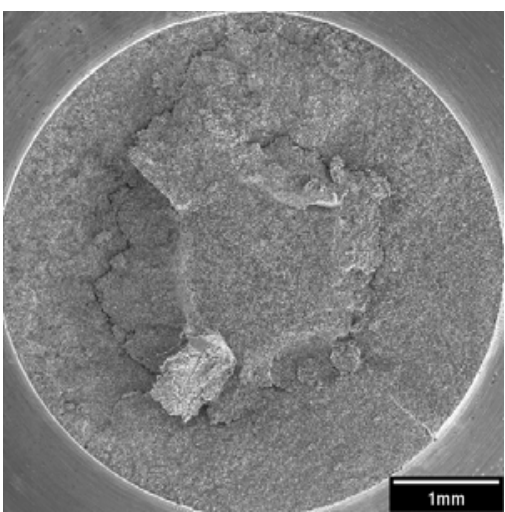

Hydrogen charged - Low Mag.

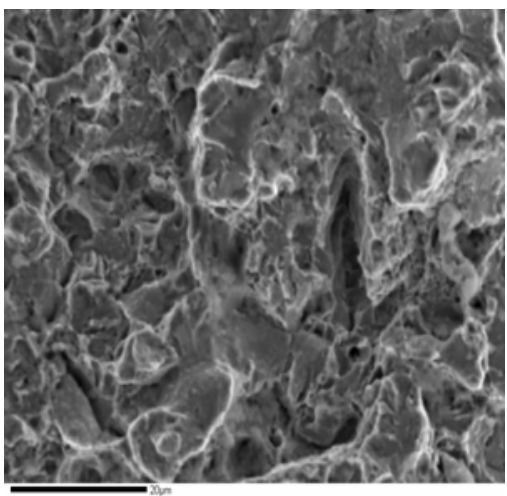

Charged Center - Quasi-Cleavage 


\section{QT 1065 - 361 HVN}

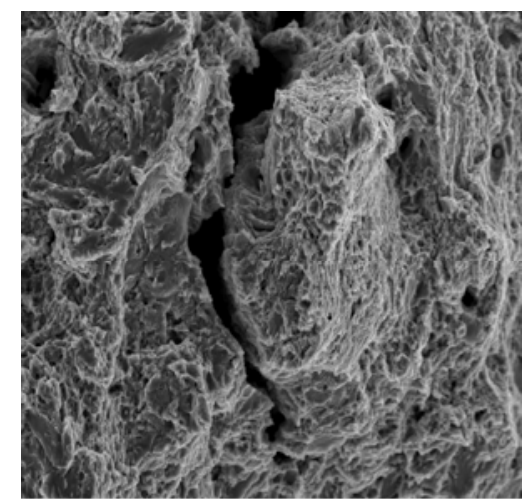

Air - MVC/Micro-cracking

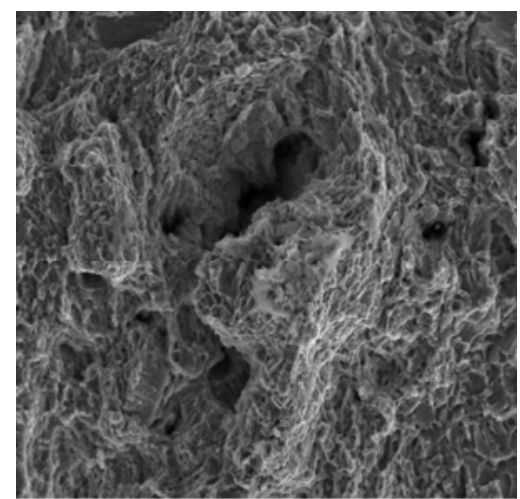

Hydrogen Charged - MVC/Micro-Cracking

\section{QT 1065 - 491 HVN}

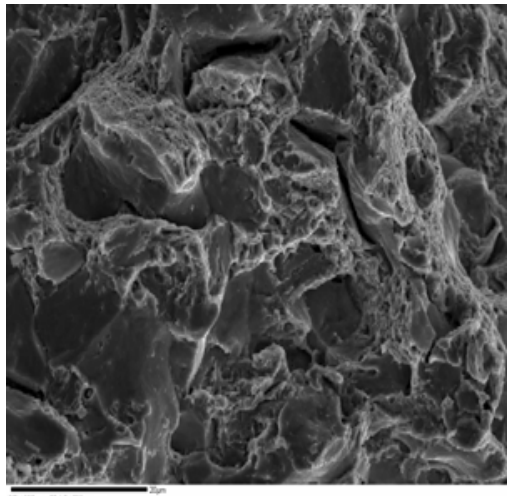

Air - Quasi-Cleavage/MVC/

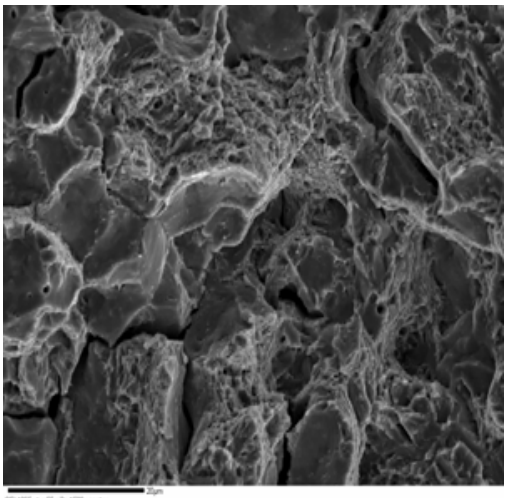

Hydrogen Charged - Same as Ai

AT 1065 - 361 \& 529 HVN (Similar Fracture)

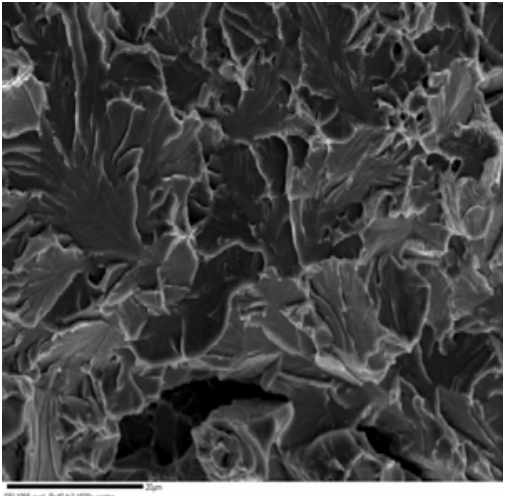

Air - Cleavage

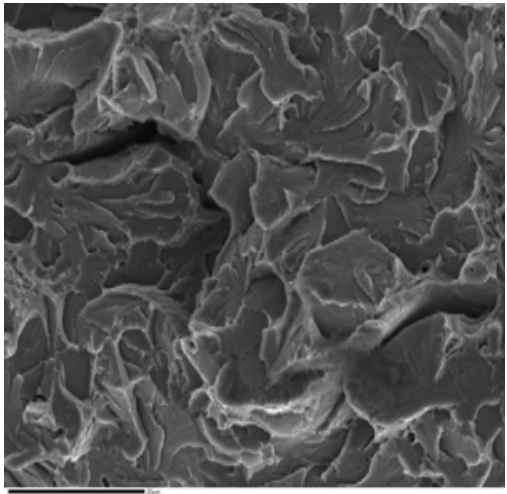

Hydrogen Charged - Cleavage 


\section{QT 1095 - 334 HVN}

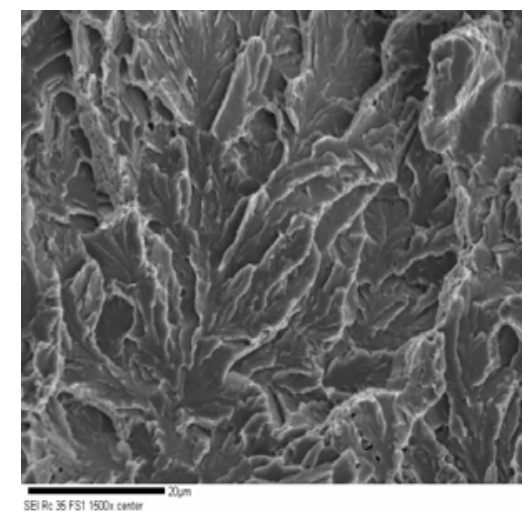

Air - Cleavage

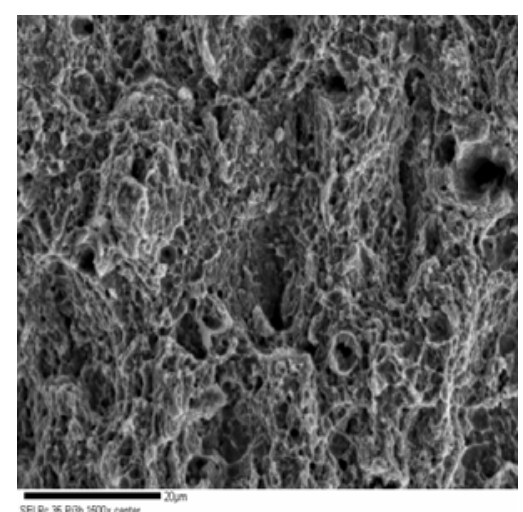

Hydrogen Charged - MVC

\section{QT 1095 - 481 HVN}

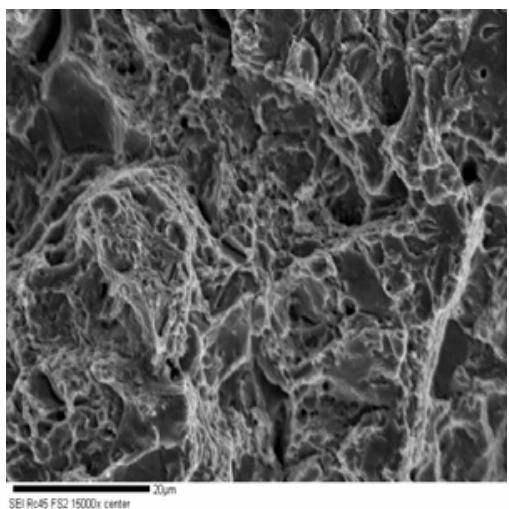

Air - Quasi-Cleavage

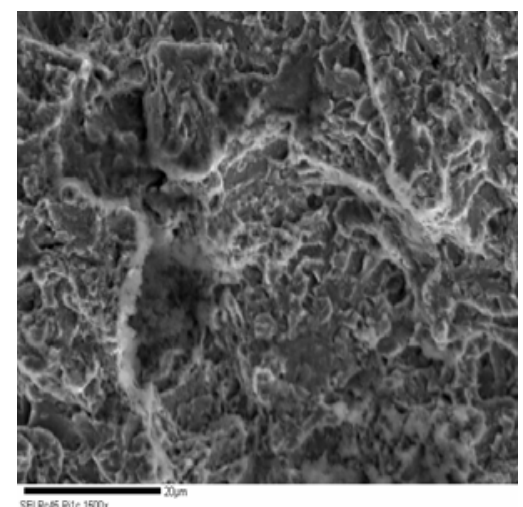

Hydrogen Charged - Quasi-Cleavage

\section{AT 1095 - 435 \& 483 HVN (Similar Fracture)}

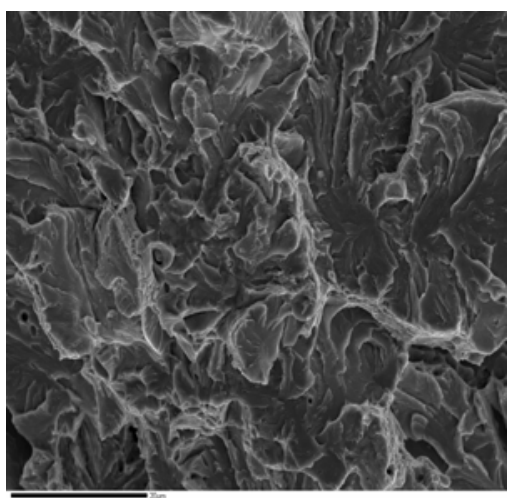

Air - Cleavage

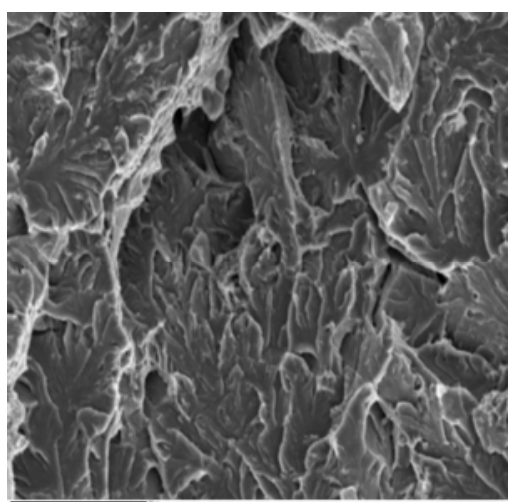

Hydrogen Charged - Cleavage 
Dual Phase 4037 - 389 \& 473 HVN (Similar Fracture)

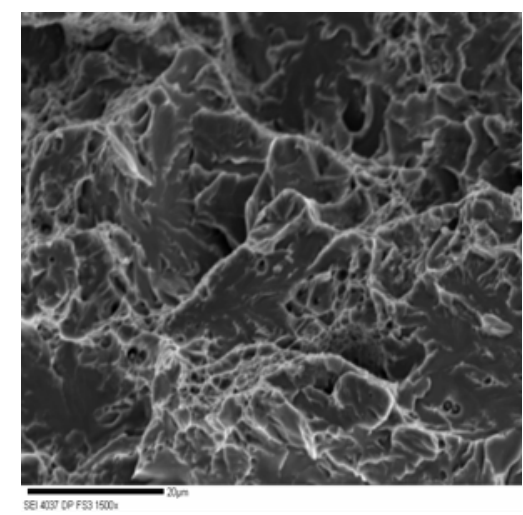

Air - Quasi-Cleavage

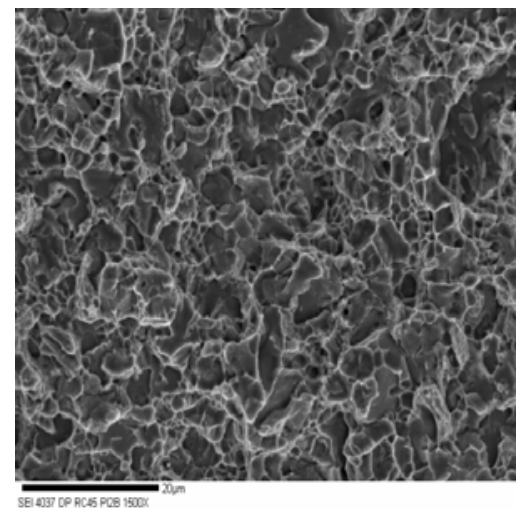

Hydrogen Charged - MVC/Intergranular 